\title{
Sensing and Vision-based Control of a Spherical Motion Platform
}

\author{
by
}

\section{Suhang Zhou}

\author{
A thesis submitted to \\ the Faculty of Graduate Studies and Postdoctoral Affairs \\ in partial fulfilment of \\ the requirements for the degree of \\ Master of Applied Science \\ in
}

Mechanical Engineering
Carleton University
Ottawa, Ontario, Canada

January 2013

Copyright (C)

2013 - Suhang Zhou 
Library and Archives

Canada

Published Heritage

Branch

395 Wellington Street

Ottawa ON K1A ON4

Canada
Bibliothèque et

Archives Canada

Direction du

Patrimoine de l'édition

395 , rue Wellington

Ottawa ON K1A ON4

Canada
Your file Votre référence

ISBN: 978-0-494-94279-6

Our file Notre référence

ISBN: $978-0-494-94279-6$
NOTICE:

The author has granted a nonexclusive license allowing Library and Archives Canada to reproduce, publish, archive, preserve, conserve, communicate to the public by telecommunication or on the Internet, loan, distrbute and sell theses worldwide, for commercial or noncommercial purposes, in microform, paper, electronic and/or any other formats.

The author retains copyright ownership and moral rights in this thesis. Neither the thesis nor substantial extracts from it may be printed or otherwise reproduced without the author's permission.
AVIS:

L'auteur a accordé une licence non exclusive permettant à la Bibliothèque et Archives Canada de reproduire, publier, archiver, sauvegarder, conserver, transmettre au public par télécommunication ou par l'Internet, prêter, distribuer et vendre des thèses partout dans le monde, à des fins commerciales ou autres, sur support microforme, papier, électronique et/ou autres formats.

L'auteur conserve la propriété du droit d'auteur et des droits moraux qui protege cette thèse. $\mathrm{Ni}$ la thèse ni des extraits substantiels de celle-ci ne doivent être imprimés ou autrement reproduits sans son autorisation.
In compliance with the Canadian Privacy Act some supporting forms may have been removed from this thesis.

While these forms may be included in the document page count, their removal does not represent any loss of content from the thesis.
Conformément à la loi canadienne sur la protection de la vie privée, quelques formulaires secondaires ont été enlevés de cette thèse.

Bien que ces formulaires aient inclus dans la pagination, il n'y aura aucun contenu manquant. 


\section{Abstract}

This thesis presents the development and real-time implementation of a novel visionbased orientation sensor (VOS) for the absolute measurement of Atlas technology demonstration platform (Atlas TDP). The VOS takes the image of a set of binary code markers mounted on the surface of the Atlas TDP sphere. It then processes the image and obtains an estimation of the orientation of the sphere. A nonlinear quaternionbased kinematic controller is modified and implemented on the Atlas TDP using the orientation feedback from the VOS. In this controller, a gain scheduling scheme is developed to meet the requirements of fast response and small steady state error of the TDP system. In order to maintain the continuity of the orientation data flow in case the VOS misidentifies markers, a sensor fusion algorithm using unscented Kalman filter (UKF) is developed. This algorithm can also be used to generate more data samples between VOS measurements to match higher control system rates. Experiments were conducted to verify the performances of the VOS, the nonlinear quaternion-based kinematic controller, and the UKF sensor fusion algorithm. The details of the tests are given in this thesis, as well as the discussion of the results. 
I would like to dedicate this work to my fiancé Yiqing $\mathrm{Lu}$ and my parents Xinyan Zhou, Min Chen for their endless blieve and love to me. 


\section{Acknowledgments}

I would like to acknowledge the following individuals for their aid and guidance:

Carleton University current P.H.D. candidate, Kyle Chisholmm, who helped me with the camera calibration and the understanding of UKF. I gained great help from your Matlab code and the face to face talking with you. Your patience and kindness contribute a lot to the success of my research.

2012 Carleton University M.A.Sc. graduate, Kyle Klumper, who helped me in operating the Optotrak and going through the previous CUSP work.

Carleton University Software and Network Specialist, Bruce K. Johnston who helped me in setting up the real-time computers.

Carleton University current M.A.Sc. candidate, Menggu He, who provided help in several experiments and discussions on several technical issues.

Finally, my supervisors, Carleton University, Professor Ahmadi and Professor Langlois for your knowledge, support and encouragement in every section of the past two years's research. 


\section{Table of Contents}

$\begin{array}{ll}\text { Abstract } & \text { ii }\end{array}$

Acknowledgments $\quad$ iv

Table of Contents $\quad \mathbf{v}$

List of Acronyms viii

List of Symbols $\quad$ x

1 Introduction 1

1.1 Atlas Platform Background .................. 1

1.1.1 Atlas Lite Platform . . . . . . . . . . . . . . . . 3

1.1 .2 Atlas TDP ..................... 6

1.1.3 Sensor Fusion . . . . . . . . . . . . . . . . . . . 14

1.1.4 Quaternions ....................... 15

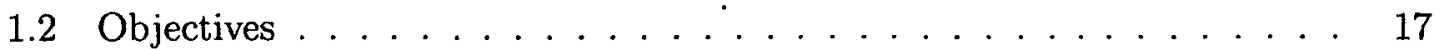

1.3 Contribution . . . . . . . . . . . . . . . 18

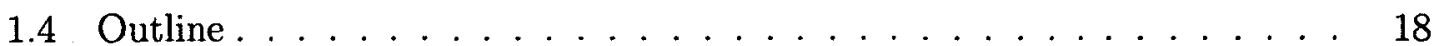

2 Visual Orientation Sensor $\quad 20$

2.1 Marker Design and Testing . . . . . . . . . . . . . . . 23

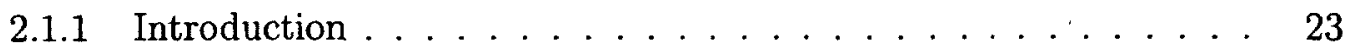


2.1 .2 Original VOS Design . . . . . . . . . . . . . 26

2.1.3 Final Design: Binary Code Markers . . . . . . . . . . . . . 28

2.1.4 Obtaining the Marker Positions in the Sphere Frame . . . . . 33

2.2 Camera Calibration . . . . . . . . . . . . . . . 35

2.2 .1 Introduction . . . . . . . . . . . . 35

2.2.2 Intrinsic Parameters . . . . . . . . . . . . . . 37

2.2.3 Obtaining the Intrinsic Parameters . . . . . . . . . . . . . 41

2.2.4 Extrinsic Parameters . . . . . . . . . . . . . . . . 43

2.2.5 Adjustment of the Camera Settings . . . . . . . . . . . 51

2.3 VOS Real-time Implementation . . . . . . . . . . . . . . . . . 54

2.3.1 Image Processing for Marker Identification . . . . . . . . . . . . 54

2.3.2 Rotation Matrix Calculation . . . . . . . . . . . . 57

2.3.3 VOS Testing on the Real-time Platform . . . . . . . . . 63

2.3.4 Conclusion . . . . . . . . . . . . . . . . 64

3 Vision-based Orientation Control $\quad 66$

3.1 Nonlinear Quaternion-based Controller . . . . . . . . . . . . 67

3.2 Controller Implementation on the LABVIEW RT Platform . . . . . . . 70

3.3 Gain Scheduling Controller . . . . . . . . . . . . . . . 75

3.4 Controller Test Results . . . . . . . . . . . . . . . . . . . . . . . 80

3.5 Discussion and Conclusion ................... 90

4 Preliminary Sensor Fusion of VOS and IOS 91

4.1 Unscented Kalman Filter . . . . . . . . . . . . . . . . . . . . . . . . . . 92

4.1 .1 Process Model . . . . . . . . . . . . . . . . 92

4.1.2 Measurement Model _. . . . . . . . . . . . . . . 95

4.1 .3 UKF Implementation Structure . . . . . . . . . . . . . . . . 96

4.2 Real-time Implementation . . . . . . . . . . . . . . . . . 102 
4.2 .1 Test Results . . . . . . . . . . . . . . . . 103

4.3 Discussion and Conclusion . . . . . . . . . . . . . . 110

5 Conclusions and Future Work $\quad 111$

5.1 Conclusions . . . . . . . . . . . . . . . . . 111

5.1.1 Vision-based Orientation Sensor . . . . . . . . . . . 111

5.1.2 Vision-based Orientation Control . . . . . . . . . . . . 112

5.1.3 Sensor Fusion of VOS and IOS . . . . . . . . . . . . . . 112

5.2 Future work . . . . . . . . . . . . . . . . 113

List of References $\quad 114$

$\begin{array}{ll}\text { Appendix A } & 118\end{array}$

$\begin{array}{lr}\text { Appendix B } & 123\end{array}$ 


\section{List of Tables}

2.1 The test results of binary marker design. . . . . . . . . . . . . 32

2.2 The Intrinsic Parameters from OPENCV and MATLAB . . . . . . 42

2.3 Coordinates of $P$ of the 4 Squares Pattern in image frame . . . . . 46

2.4 Coordinates of $P$ in the Optotrak frame O1. . . . . . . . . 48

2.5 Coordinates of $P$ in the sphere frame $O 2 \ldots \ldots \ldots \ldots$

2.6 Calculated Coordinates of $\mathrm{P}$ versus the Original Coordinates . . . . . . 52

2.7 Light Settings . . . . . . . . . . . . . . . . . . . . . . 53

2.8 Camera Settings . . . . . . . . . . . . . . . . . . 54

3.1 RMS error of single-DOF sinusoidal response without time shift . . . . 83

3.2 Steady state error of single-DOF step response . . . . . . . . . . . . . 84

3.3 RMS error and maximum error of single-DOF sinusoidal response . . . 84

3.4 Steady State Error of Multi-DOF Step Response . . . . . . . . . . . . 84

3.5 RMS Error and Maximum Error of Multi-DOF Sinusoidal Response . . 87

3.6 RMS error and maximum error of a flight simulation . . . . . . . . . 88

4.1 3DM-GX1 Specifications . . . . . . . . . . . . . . . . 102 


\section{List of Figures}

1.1 The initial concept of Atlas platform. . . . . . . . . . . . 2

1.2 Actuation design for Atlas platform. . . . . . . . . . . . . . 3

1.3 Atlas Lite demonstration model. . . . . . . . . . . . . . . . . . 4

1.4 The Gearbox and Motor housing with a mounted omniwheel for Atlas Lite. . . . . . . . . . . . . . . . . . 5

1.5 The $3 \mathrm{D}$ rendering of full-scale Atlas platform. . . . . . . . . . . 8

1.6 Atlas TDP platform . . . . . . . . . . . . . . . . 9

1.7 The Base strcture of TDP. . . . . . . . . . . . . . . 10

1.8 The Driving mecanum wheel design. . . . . . . . . . . . . . 11

1.9 The System View of the TDP. . . . . . . . . . . . . 11

1.103 DMG-GX1 sensor. . . . . . . . . . . . . . . . . . . . 12

1.11 The PID control Sturcture for Atlas TDP. . . . . . . . . . . . . . 14

2.1 The marker system layout. . . . . . . . . . . . . . . . . . 22

2.2 single colour markers on the desktop model. . . . . . . . . . . . . . . . 24

2.3 The mixed colour marker design. . . . . . . . . . . . . . . . . . 24

2.4 The ARtag markers. . . . . . . . . . . . . . . . . . 25

2.5 The design of number 127 binary marker. . . . . . . . . . . . 25

2.6 Diagram illustrating calculation of the marker number. . . . . . . . . . 29

2.7 Marker boards for marker design testing. . . . . . . . . . . . . . . . . . 31

2.8 CRS manipulator. . . . . . . . . . . . . . . . . . . . . . . . . . . . 31 
2.9 The Optotrak measurement system. . . . . . . . . . . . . . 34

2.10 Position sensor Optotrak. . . . . . . . . . . . . . . 36

2.11 Demonstration of the process of measuring $P_{s} \ldots \ldots \ldots$

2.12 The transformation between camera frame and global frame [1]. . . . . 37

2.13 A pinhole camera model. . . . . . . . . . . . . . 37

2.14 Chessboard for Camera Calibration. . . . . . . . . . . . . . . . . 41

2.15 Different positions of the check board . . . . . . . . . . . 43

2.164 squares feature extracting pattern. . . . . . . . . . 45

2.17 Image of the square pattern. . . . . . . . . . . . . 46

2.18 Demonstration of the Optotrak frame and the sphere frame. . . . . . 48

2.19 Image quality before and after the camera parameters setting . . . . . 53

2.20 Flow Diagram of VOS. . . . . . . . . . . . . . . 55

2.21 Demonstration of centre compensation. . . . . . . . . . 56

2.22 Demonstration of pixel selection. . . . . . . . . . . 58

2.23 Examples of marker identification: Number 87 and Number 110. . . . 59

2.24 Camera Model Demonstration. . . . . . . . . . . . . . 61

2.25 LABVIEW VOS program GUI. . . . . . . . . . . . . . 63

2.26 VOS and IOS measurement of a rotation of $120^{\circ} \ldots \ldots \ldots 4$

3.1 Block diagram of the orientation controller for the Atlas TDP. . . . . 67

3.2 The GUI of the LABVIEW Control Program. . . . . . . . . . . . . 71

3.3 The GUI of the LABVIEW Control Program. . . . . . . . . . . . 73

3.4 Flow Chart of the TDP system over Timeline. . . . . . . . . . . . 75

3.5 Performance of $k=1$ when $e$ is large. . . . . . . . . . . 77

3.6 Performance of $k=1$ when $e$ is small. . . . . . . . . . . . . 77

3.7 Performance of $k=8$ when $e$ is large. . . . . . . . . . . 78

3.8 Performance of $k=8$ when $e$ is large. . . . . . . . . . . . . 78

3.9 Definition of the gain $k \ldots \ldots \ldots \ldots \ldots$ 
3.10 Single-DOF step response of a $10^{\circ}$ input. . . . . . . . . . . 81

3.11 Single-DOF sinusoidal response of a $10^{\circ}$ input. . . . . . . . . . . . 82

3.12 Sinusoidal response on roll of a $10^{\circ}$ input without time shift . . . . . 83

3.13 Multi-DOF step repones of a $10^{\circ}$ Input . . . . . . . . . . . 85

3.14 Multi-DOF Sinusoidal Repones of a $10^{\circ}$ Input . . . . . . . . . 86

3.15 Illustration of the small errors of VOS readings. . . . . . . . . . . 88

3.16 Response of a Flight Simulation. . . . . . . . . . . . . . . 89

4.1 The out of sequence handling of VOS data . . . . . . . . . . . 97

4.2 The flow chart of the UKF. . . . . . . . . . . . . . 101

4.3 The block diagram of the sensor fusion incorporating with the controller 103

4.4 The VOS, IOS, and fused data about the pitch axis in Test 1. . . . . 104

4.5 The VOS, IOS, and fused data about roll axis in Test 1. . . . . . . 105

4.6 The VOS, IOS, and fused data about yaw axis in Test 1. . . . . . . 106

4.7 The VOS, IOS, and fused data about the pitch axis in Test 2. . . . . 107

4.8 The VOS, IOS, and fused data about roll axis in Test 2. . . . . . . 107

4.9 The VOS, IOS, and fused data about yaw axis in Test 2. . . . . . 108

4.10 Response of a sinusoidal input of the controller in Test 3 . . . . . . . 109

A.0 6 and $4 \mathrm{~cm}$ markers on white background . . . . . . . . 120

A.-1 $4 \mathrm{~cm}$ markers on black background . . . . . . . . . . . . . 121

A.-1 $6 \mathrm{~cm}$ markers on black background . . . . . . . . . . . . . 122

A.0 All 38 images of the results of marker design tests . . . . . . . . . . 122 


\section{List of Acronyms}

\begin{tabular}{ll}
\hline Acronyms & Definition \\
\hline VOS & Visual Orientation Sensor \\
TDP & Technology Demonstration Platform \\
IOS & Interial Orientation Sensor \\
UKF & Unscented Kalman Filter \\
DOF & Degree of Freedom \\
CUSP & Carleton University Simulation Project \\
RTOS & Real-time Operating System \\
FPGA & Field-programmable Gate Array \\
PID & proportionalintegralderivative \\
EKF & extended Kalman filter \\
& unscented transformation \\
RT &
\end{tabular}




\begin{tabular}{ll} 
AR & Augmented Reality \\
RMS & root mean square \\
OpenCV & open source computer vision library \\
GUI & graphical user interface \\
ARW & angular random walk \\
NAN & not a number \\
\hline
\end{tabular}




\section{List of Symbols}

\begin{tabular}{|c|c|}
\hline Symbols & Definition \\
\hline$J$ & Jacobiann Matrix \\
\hline$\Omega$ & Anglar Velocity of the sphere \\
\hline$\omega$ & Angular Velocity of the motors \\
\hline$r$ & Radius of the mecanum wheels \\
\hline$R$ & Radius of the sphere \\
\hline$\alpha$ & Castor orientation angle \\
\hline$\phi_{i}$ & Anlge between motor $i$ to each of the motors $A, B, C$ \\
\hline$\theta$ & $\begin{array}{l}\text { Orientation Angle of the mecanum wheels from the equator } \\
\text { of the TDP sphere }\end{array}$ \\
\hline$q$ & unit quaternion \\
\hline$q_{0}, q_{1}, q_{2}, q_{3}$ & Four components of unit quaternion \\
\hline$R_{\text {rot }}$ & Rotation Matrix \\
\hline$\omega_{\text {crs }}$ & Angular Velocity of the CRS robot \\
\hline
\end{tabular}




\begin{tabular}{|c|c|}
\hline$\omega_{\text {sphere }}$ & Maximum Angular Velocity of the sphere \\
\hline$P_{s}$ & Positions of markers in the sphere frame \\
\hline$P_{w}$ & Positions of markers in the world frame \\
\hline$P_{s 1}$ & $\begin{array}{l}\text { Coordinates of markers in the sphere frame measured in } \\
\text { the first round in frame } F_{1}\end{array}$ \\
\hline$P_{f 2}$ & $\begin{array}{l}\text { Coordinates of markers in the second round of measure- } \\
\text { ment in frame } F_{2}\end{array}$ \\
\hline$H$ & $\begin{array}{l}\text { Transformation matrix from the second round of measure- } \\
\text { ment of } P_{s} \text { to the first round measurement }\end{array}$ \\
\hline$f_{x}, f_{y}$ & Focal lengths of the camera along $x$ and $y$ axes \\
\hline$C_{x}, C_{y}$ & Coordinates of the center of the camera frame \\
\hline$k$ & Distortions of the lens \\
\hline$[X, Y]^{T}$ & Position of point $P$ in the global frame \\
\hline$[x, x]^{T}$ & Position of point $P$ in the image frame \\
\hline$k_{1}, k_{2}, k_{3}$ & Three coefficients of radial distortion \\
\hline$\left[X_{\text {correct }}, Y_{\text {correct }}\right]^{T}$ & Corrected coordinates of point $P$ from distortions \\
\hline$p_{1}, p_{2}$ & Coefficients of the tangential distortions \\
\hline$P_{\text {camera }}$ & Vector containing intrinsic parameters \\
\hline$H_{r}$ & $\begin{array}{l}\text { Rotation matrix describing the transformation from global } \\
\text { frame to the image frame }\end{array}$ \\
\hline
\end{tabular}




\begin{tabular}{|c|c|}
\hline$H_{t}$ & $\begin{array}{l}\text { Translation matrix describing the transformation fron } \\
\text { global frame to the image frame }\end{array}$ \\
\hline$r_{i j}$ & Elements of the homegeneous transformation matrix $E$ \\
\hline$P_{i}$ & Coordinates of $P$ at the 10 different pattern positions \\
\hline$\hat{x}, \hat{y}, \hat{z}$ & Unit vectors of the rotation matrix from frame $O_{2}$ to $O_{1}$ \\
\hline E & Extrinsic parameters matrix \\
\hline$(u, v)$ & Location of markers in the image frame \\
\hline$\hat{x}, \hat{y}$ & Distorted coordinates in the image frame \\
\hline$\left(\delta x_{t}\right)_{k},\left(\delta y_{t}\right)_{k},\left(\delta x_{r}\right)_{k}$, and $\left(\delta y_{r}\right)_{k}$ & Distortion Factors \\
\hline$P_{c}$ & Coordinates of $P$ in the camera frame \\
\hline$Q_{c}^{w}$ & Translation matrix from $P_{w}$ to $P_{c}$ \\
\hline$q_{k}$ & Quaternion at time step $k$ \\
\hline$w_{k}$ & Process noise at step $k$ \\
\hline$T_{s}$ & Sampling period \\
\hline$r_{k}$ & Rotation from step $k-1$ to $k$ \\
\hline$\theta$ & Rotation angle of the sphere from step $k-1$ to $k$ \\
\hline$n_{b}$ & Bias of the IOS \\
\hline$n_{\omega}$ & Noise of IOS \\
\hline$V_{\omega}$ & IOS measurement noise \\
\hline
\end{tabular}


Axis-angle vector

$\hat{a}$

Norm of $\vec{a}$

$Z_{k}$

Measurement state

${ }^{i m} P_{i}$

Markers' locations in the image frame for the orientation computation

$\sigma_{i m}^{2}$

Covariance of the marker location measurement in the image frame

$\sigma_{s}^{2}$

Covariance of the marker location measurement in the sphere frame

$R_{m}$

Covariance matrix of the VOS measurement model

Updated measurement at step $k$

Rotation from step $l$ to $k$

A priori estimate of the state at step $k$

$\hat{q}_{l}^{+}$

A posteriori estimation of the state at step $l$

$q^{-1}$

Inverse of the quaternion

$\tilde{a}^{(i)}$

Sigma point in axis-angle format

$P_{k-1}^{+}$

A posteriori covariance from step $k-1$

$\hat{a}^{(i)}$

Sigma point in quaternion format

$e_{q}^{i}$

Error rotation

$\bar{q}_{k}$

Mean orientation 


\begin{tabular}{ll}
$\bar{e}_{a}$ & Mean error rotation \\
$\bar{q}_{k_{t+1}}$ & Mean orientation at the iteration step $t+1$ \\
$Q_{i o s}$ & Covariance of the IOS measurement noise \\
$R_{v o s}$ & Covariance of VOS measured orientation \\
$\hat{Z}_{k}$ & Mean estimate of the VOS measurement of orientation at \\
$P_{z}$ & step $k$ \\
& Covariance estimate of the VOS measurement of orienta- \\
$K_{k}$ & tion at step $k$ \\
$V_{k}$ & Kalman gain at step $k$ \\
$\hat{q}_{k}^{+}$ & Innovation error rotation \\
$P_{k}^{+}$ & A posteriori state estimate mean \\
\hline
\end{tabular}




\section{Chapter 1}

\section{Introduction}

\subsection{Atlas Platform Background}

Motion platforms are widely used for a variety of applications, especially for training simulations such as flight simulation [2]. The conventional Stewart platform hexpods with six degree of freedom (DOF) is the most common used motion platform for the land, sea, and air vehicle simulation. However, the motions of Stewart platform are tightly coupled so that the platform workspace are small $\left(30^{\circ}\right.$ or $45^{\circ}$ around each direction) compared to the overall platform size. Consequently, the Stweart platform may not meet the requirements of some special applications, such as military flight simulation. It is widely accepted that the performance of a simulation platform can be improved if the range of motion is larger. Researches have been conducted on developing new motion platforms with larger range of motion over the Stewart platform. Three existing simulation platforms are Desdemona [3], Eclipse [4] and CyberMotion [5]. In Carleton University, another motion platform was designed and developed named as Atlas platform. An Atlas platform is a 6-DOF multi-functional simulator designed by Carleton University Simulator Project (CUSP) team within the Department of Mechanical and Aerospace Engineering as an improvement on typical Stewart platform by producing unlimited rotational motion. CUSP is a $4^{\text {th }}$ year project introduced in 
2002 at the Departments of Mechanical and Aerospacing Engineering and System and Computers Engineering. The objective of CUSP is to make the new simulator capable of simulating a range of vehicle types (air, sea, land) in 6-DOF. The intial concept of the Atlas platform is shown in Figure 1.1 [6], where the main components are a sphere with an inner cockpit large enough for a single occupant. The sphere is supported by an outer structure incorporating the use of omniwheels to allow the sphere to rotate freely [6]. This Atlas concept was chosen because it can provide the most innovative and effective way to simulate any type of motion, either rotation or translation. It is offering the ability of infinite rotation in any direction, which cannot be achieved with a traditional Stewart platform.

The Atlas sphere is actuated by three omnidirectional friction wheels (omniwheels)

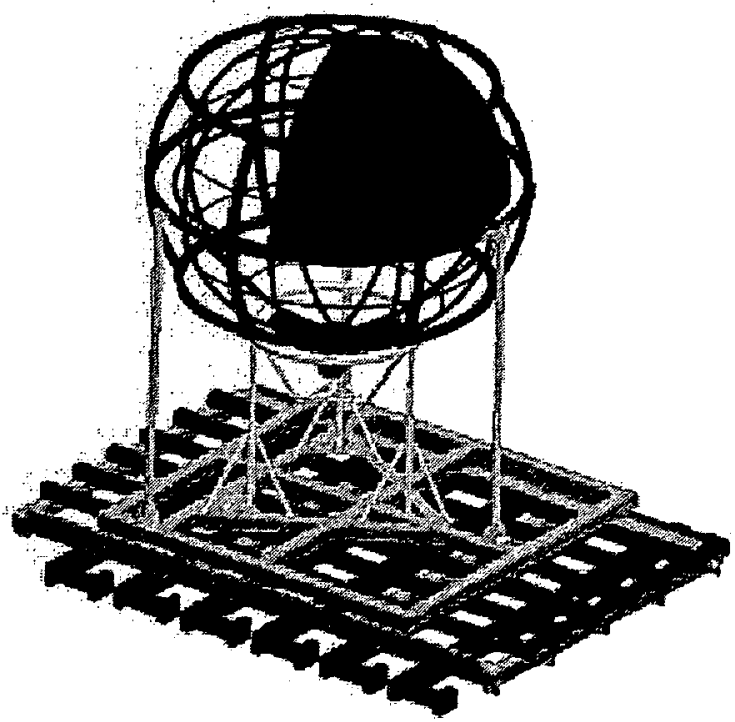

Figure 1.1: The initial concept of Atlas platform.

which are oriented to give three motions: roll, pitch, and yaw. These wheels are mounted to the surpporting frame in Figure 1.1. A Pro-Engineer model of the actuation method can been seen in Figure 1.2 [6]. These omnidirectional wheels will be further introduced in the following sections. Based on the initial concept, two Atlas 
models were designed and manufactured: a tabletop platform (Atlas Lite) and a halfscale demonstration platform (Atlas TDP).

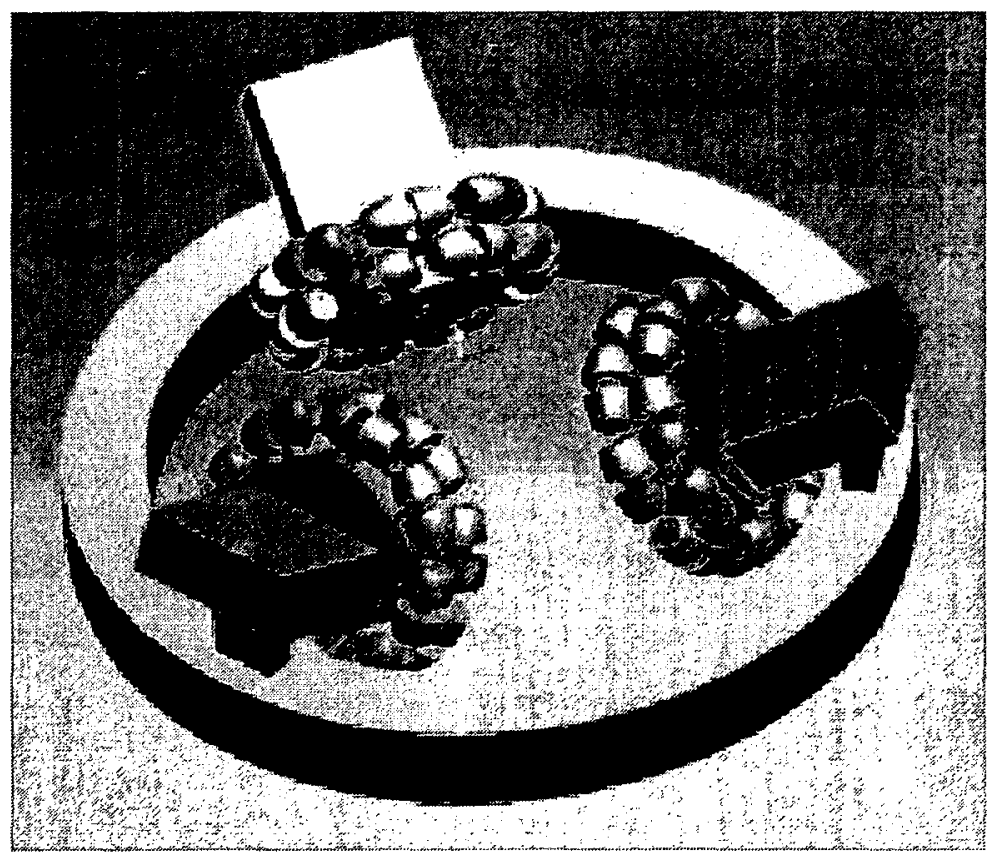

Figure 1.2: Actuation design for Atlas platform.

\subsubsection{Atlas Lite Platform}

\section{Actuation of Atlas Lite}

In the academic year of 2004-2005, the CUSP group designed the tabletop concept demonstrator Atlas Lite as shown in Figure 1.3 [7]. The rotational stage is physically constructed of a sphere whose diameter is 8 inches and three dual-row omnidirectional wheels maintaining continuous contact with the sphere [7]. An omniwheel develops traction in one direction and allows passive motion in another. Therefore, by implementing the omniwheels in the correct configuration, one is able to produce all three rotational DOF with relative ease [6]. Each of these omniwheels has a diameter 
of 1.5 inch and thickness of 1.1 inch, and their rollers are oriented at $90^{\circ}$ to the circumference of the wheels. Each wheel is actuated by a Tamiya 6-Speed Gearbox H.E. motor which is capable of providing a maximum speed of $132 \mathrm{rpm}$ and torque of 0.9 in-lbf as shown in Figure 1.4 [6]. The three motors are actuated with different angular velocities according to the command to drive the sphere toward any angular position in 3D space.

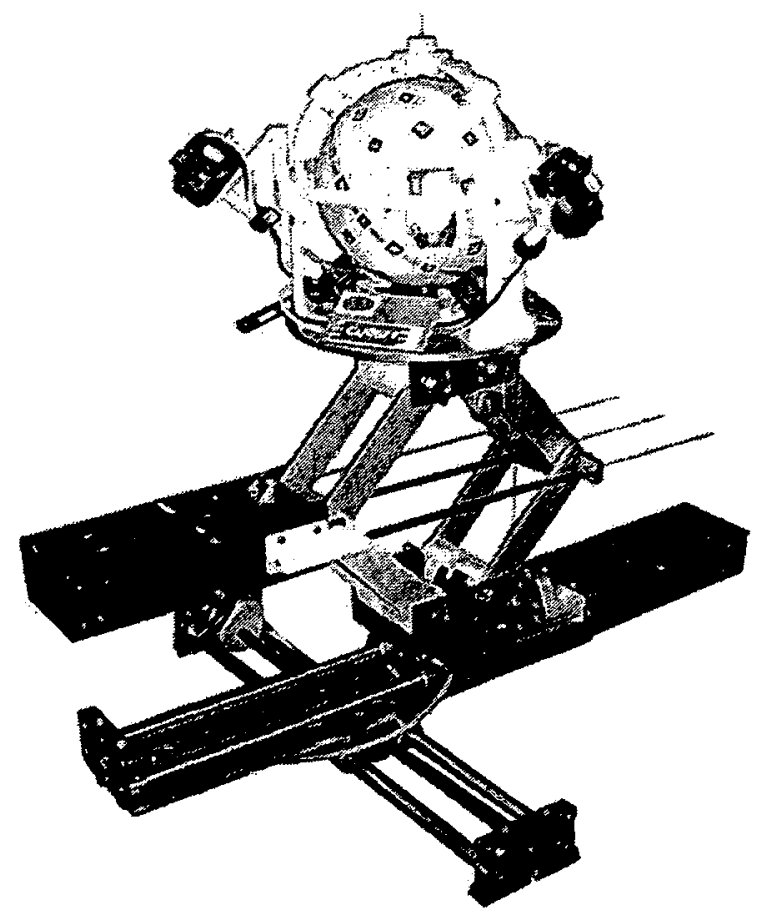

Figure 1.3: Atlas Lite demonstration model.

\section{Atlas Lite Sensing}

After the innovative design of the actuation method of Atlas, for the purpose of motion control, both the kinematic and dynamic models were developed to describe the relationship between the required simulated motion repsonse of the sphere and 


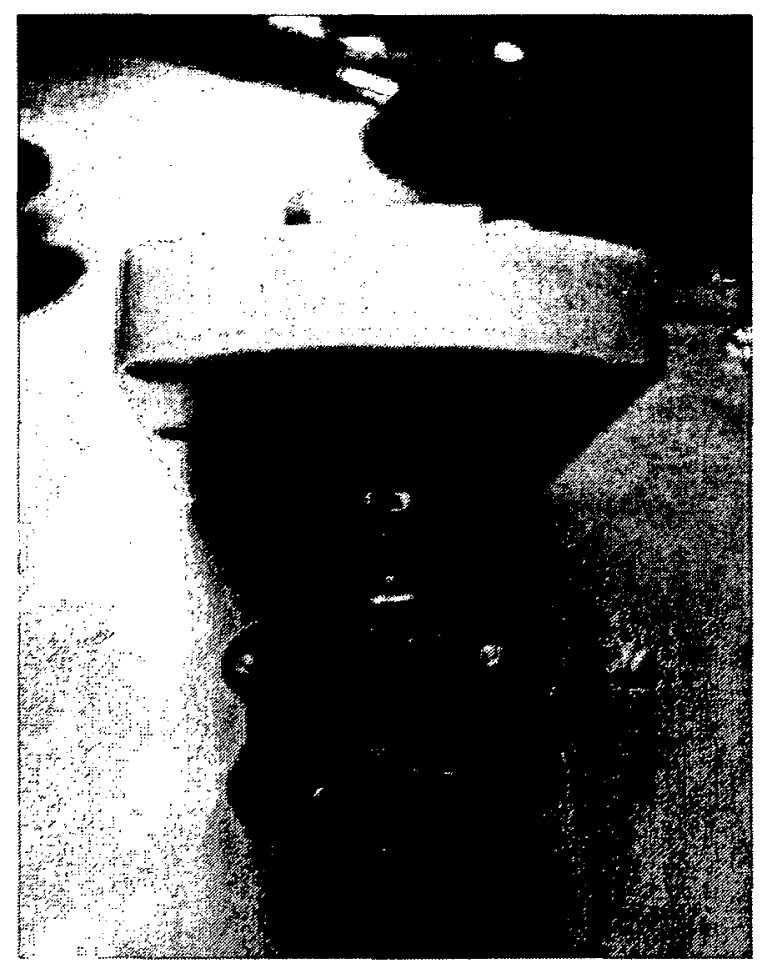

Figure 1.4: The Gearbox and Motor housing with a mounted omniwheel for Atlas Lite.

the actuators during the years from 2004-2006. The details of the kinematic and dynamic models can be found in [8]. An independent measurement of the decoupled rotation is needed due to the slippage between the sphere and the wheels. The sensors include the motor shaft encoders, the inertial orientation sensor (IOS), and the visual orientation sensor (VOS). The shaft encoders are attached to each of the omniwheel shafts to measure the angular displacement of the shaft. The IOS has a high refresh rate and is sensitive to quick changes in motion. It uses three gyroscopes and a tri-axis accelerometer to sense the motion of the sphere as the occupant would feel it [9]. However, this sensor has an inherent flaw as the electronic components are subject to drift. The orientation errors accumulate over time as they are computed by integrating the angular velocity. To correct the errors, the VOS was developed as an absolute sensor working in parallel with the IOS. The main component of the VOS is a 
digital camera pointing at the sphere to identify the locations of featured dots on the surface of the sphere. The orientation of the sphere can be obtained by comparing the locations of the featured dots before and after a certain rotation. The VOS has a much slower update rate compared to the IOS since it is limited by the maximum frame rate of the camera. However, it can be more accurate as the resulting absolute measurement.

\section{Feeback Control of Atlas Lite}

A feedback control loop is used to correct for error, noise, and disturbances found in the system, mainly caused by the slippage from the contact of the omniwheels and the sphere [9]. Any of the three sensor system can be the feedback source of the control loop, however, they all have their respective drawbacks and advantages. To maximize the accuracy of the feedback orientation measurement of the sphere, a combination of the sensors would be possible to use.

Atlas Lite was completed and achieved good performance of controlling the sphere [2]. As the Atlas Lite was a low-cost platform with several mechanical, electrical, and real-time control limitations, the entire project moved on to the next stage of development: Realization of the Technology Demonstration Platform (TDP) to demonstrate the main technological modules related to actuation, sensing, and control.

\subsubsection{Atlas TDP}

\section{Actuation of TDP}

In the academic year of 2006-2007, the idea of the Atlas TDP was proposed as a transition platform between the Atlas Lite and the full-scale simulator (Figure 1.5 [10]) 
as shown in Figure 1.6. The TDP follows the same design concept of the Atlas platform. As it is actuated by mecanum wheels of a type similar to omnidirectional wheels mounted on the base design shown in Figure 1.7. Instead of omniwheels, mecanum wheels are used that have castors oriented at $45^{\circ}$ and offer the potential of less impact and smoother motion. The sensors still include the shaft encoder, IOS, and VOS. It also continues to use a feedback controller for the orientation. On the other hand, compared with the Atlas Lite, the changes of the TDP are as following:

1. The TDP sphere has a diameter of 4 feet;

2. The TDP sphere is actuated by mecanum wheels (Figure 1.8) as mentioned;

3. A real-time operating system is used to implement the feedback control and actuation system;

4. The VOS design is improved for the application on the TDP;

The goal of the TDP is to make the sphere simulate the motion of an aircraft as generated from simulation software to provide a similar physical experience to that of a real aircraft for the pilot. The system structure of the ultimate Atlas platform is shown in Figure 1.9. On TDP, a pilot outside the physical platform works with a flight simulator software and data is directly linked to the TDP controller to command the motors to actuate the sphere into the right positions.

\section{Atlas Kinematics}

A kinematic model was developed for Atlas platform to convert the angular velocity of the sphere to the angular velocity of the three motors. It is derived by assuming zero slippage betweent the mecanum wheels and the sphere, and a constant nominal contact point between the sphere and each mecanum wheel. The kinematics of Atlas 


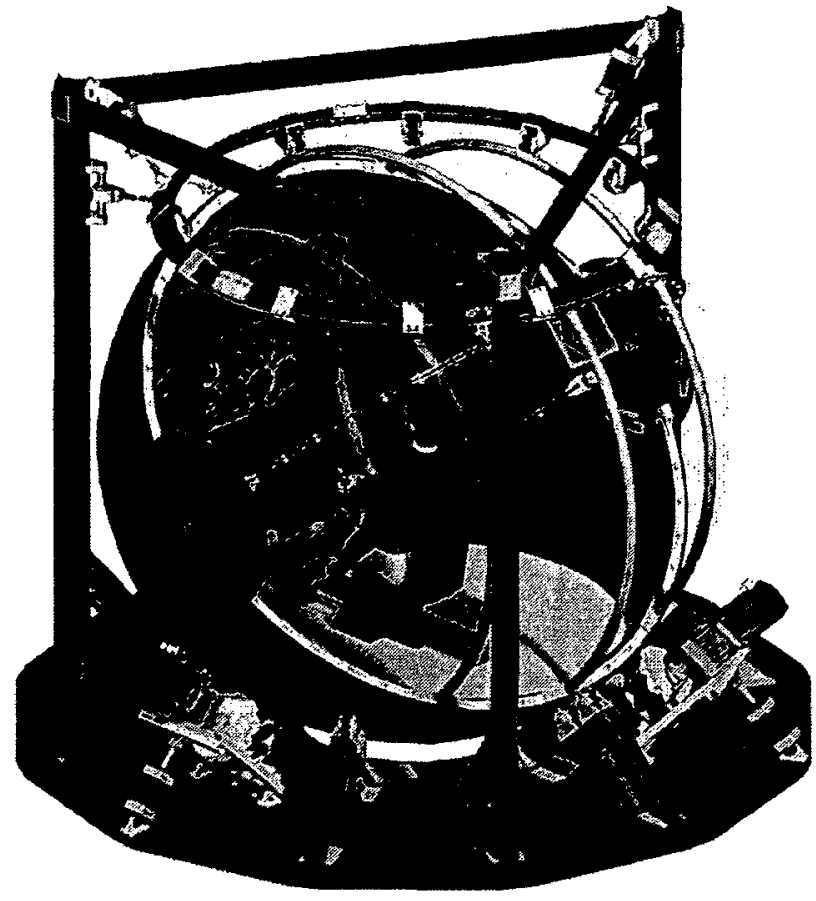

Figure 1.5: The 3D rendering of full-scale Atlas platform.

can be expressed by Equation 1.1 and 1.2 [11], where the $J$ is called Jacobian which can be seen in Equation (1.2) [11] below, $\Omega$ denotes the angular velocity of the sphere and $\boldsymbol{\omega}$ denotes the angular velocity of the motors.

$$
\Omega=J_{\omega}^{-1} \omega
$$

The Jacobian matrix is constant given by

$$
J_{\omega}=\frac{r}{R}\left[\begin{array}{ccc}
-\sin \theta_{1} & -\tan \alpha & \cos \theta_{1} \\
\sin \phi_{2} \tan \alpha-\sin \theta_{2} \cos \phi_{2} & -\sin \phi_{2} \sin \theta_{2}-\cos \phi_{2} \tan \alpha & \cos \theta_{2} \\
\sin \phi_{3} \tan \alpha-\sin \theta_{3} \cos \phi_{3} & -\sin \phi_{3} \sin \theta_{3}-\cos \phi_{3} \tan \alpha & \cos \theta_{3}
\end{array}\right]^{-1}
$$




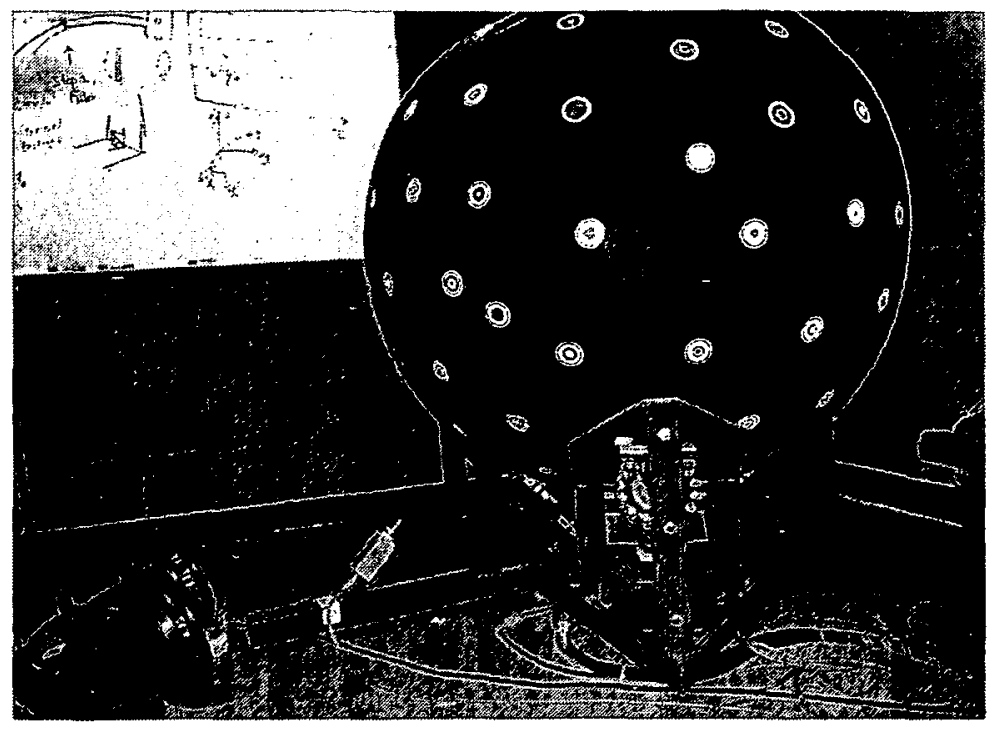

Figure 1.6: Atlas TDP platform .

where the $r$ and $R$ are the radius of the mecanum wheel and the sphere respectively; $\alpha$ representes the castor orientation angle of $\pm 45^{\circ} ; \phi$ denotes the angle between motor $A$ and each of motors $A, B, C$; and $\theta$ denotes the orientation angle of the mecanum wheels from the equator of the TDP sphere [12]. The Jacobian links the output of the feedback controller to the motor control software.

\section{TDP Sensing System}

Atlas TDP includes three main sensors (VOS, IOS, and shaft encoders) similar to Atlas Lite. The 3DM-GX1 (Figure 1.10, details in [13]) is selected as the inertial sensor housed in the sphere to allow the measurement of position, angular velocity, and acceleration of the sphere [14]. The IOS is able to produce the raw orientation data at a high frequency of around $200 \mathrm{~Hz}$, but if the angular position is selected, then the requency falls to between 60 to $70 \mathrm{~Hz}$. There are errors in yaw measurement that takes time to be compensated using the sensor's magnetometer. It is oberved that 


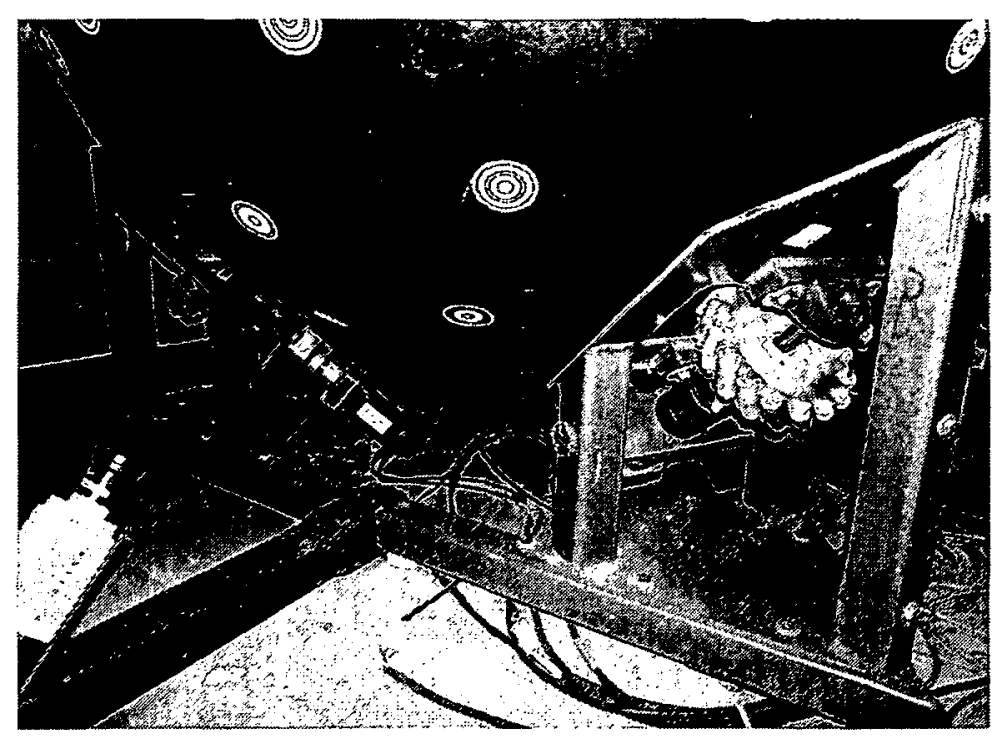

Figure 1.7: The Base strcture of TDP.

the error can become as high as $45^{\circ}$ depending upon the motion of the sphere and the environment around the IOS. Thus, the IOS cannot be selected as the only source of orientation feedback for the TDP.

A VOS system similar to that on Atlas Lite, is required for the Atlas TDP to provide an absolute measurement of the orientation of the TDP sphere. Although it follows the concept of the Atlas Lite VOS, the details of the design of the VOS for the TDP have to be considered to meet the requirements of the TDP acutation specifications and scale. A VOS was designed for Atlas Lite based on colour markers and a digital camera, and showed good performances. However, due to a limitation on the number of available distinct colour markers, it cannot be implemented on the Atlas TDP because of the larger surface area [15]. Another colour-based visiual orientation sensor was built in [16] for the application of spherical motor control, in which markers are composed of colour squares. The absolute orientation information is encoded by the colour information and position relationship of the squares in the pattern. Due to the position relationship between each markers, this method solved the problem of limited marker numbers and is capable of high-resolution measurement. However, it 


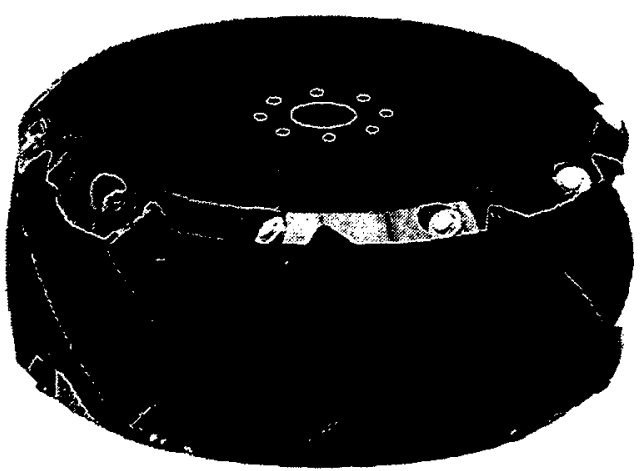

Figure 1.8: The Driving mecanum wheel design.

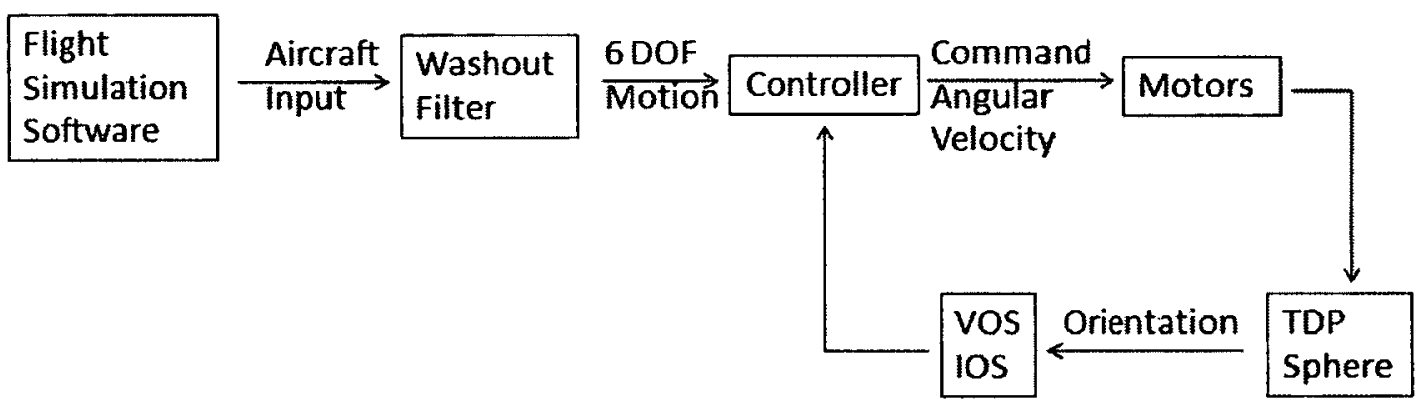

Figure 1.9: The System View of the TDP.

is difficult to design the window property for a large marker pattern over the TDP sphere surface. Instead of the colour-based pattern, [17] proposed a nonuniform spacing grid pattern (latitude and longitude lines) for extracting the characteristic points. This pattern is easy to implement but the accuracy is limited because of the equivalence in matching procedure using unequally spacing encode method. A VOS system without the colour-based markers was built for the TDP [18]. It introduces the innovative binary marker concept as the binary number could produce sufficient number of unique markers to cover the entire surface of the sphere without introducing potential misidentifications. Despite the novel design of the markers, that VOS system 


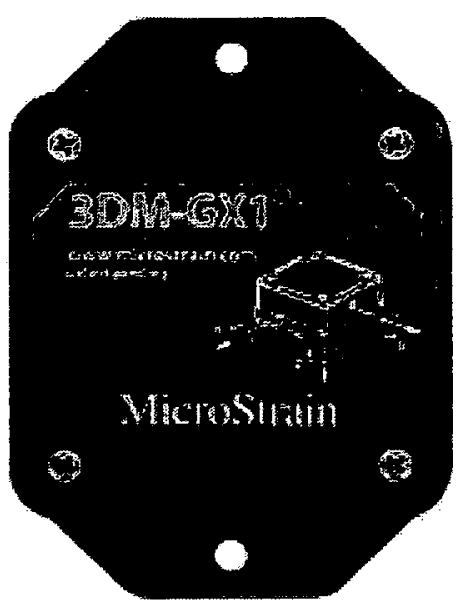

Figure 1.10: 3DMG-GX1 sensor.

did not function as expected since it lacked a stable and fast calculation algorithm. The failure was also caused by the blur on the images which was generated when the sphere was rotating at a high angular velocity. This blur has the strongest effect at the edge of the rings where the most sigificant part of the marker binary code was located. Therefore, the rate of misidentification remains high and results in the failure of the VOS. A real-time barcode reader was designed and implemented for an industrial application where the blur was succesfully removed from the image as they adopted the MATLAB function "deconvblind" [19] that uses an approach based on blind de-convolution and a maximum likelihood function. This was considered to be a potentially efficient way of removing the blur from the images. A new VOS design as well as a new software design were needed for the control of the TDP system which would be most reliable and less affected by blur or lighting conditions, which is a main objective of this research. 


\section{TDP Control Architecture}

The TDP control is similar to the Atlas Lite. Both angular position and angular velocity are used as inputs as the omni-wheel motors operate in the speed control mode $[20]$. With the introduction of a real-time operating system, the controller implementation is actually more complicated than the one for the Atlas Lite. The hardware includeing a field-programmable gate array (FPGA) data acquisition card which is compatible with the LABVIEW real-time module, motors, and the amplifiers are connected to the target computer which runs under the real-time operating system (RTOS).

An application of a simple proportional-integral-derivative (PID) controller illustrated in Figure 1.11 was designed and implemented on Atlas TDP, but was not satisfactory for an accurate and stable control [21] because of the high nonlinearity of the dynamics of the Atlas platform. A quaternion-based controller was proposed in [22], where error was defined only in the vector portion of the quaternion to get rid of the problem of singularity. The asymptotic error convergence was established in this controller. However, this controller used a linear feedback control law which would not be applied for Atlas. Therefore, a nonlinear quaternion-based controller would be the desirable plan. As lacking of an accurate dynamic model of Atlas, the controller had to be based on the kinematics. Several nonlinear quaternion-based controllers which might fulfill the requirements in Atlas were designed for the orientation controller and had sound verification of the global asymptotic stability by using Lyapunov function $[23,24]$. The controller [23] requires no information about the system such as the moment of inertia and feeds the attitude error in unit quaterion format. But it was designed for torque control while Atlas requires speed control of the motors. In [24], a comprehensive study was done on the controllers of model-dependent and model-independent, unit quaternion feedback and vector quaternion feedback. Not 
much differences were observed in terms of the performances of each controller. Similar to the controller in [23], this controller was also torque based which did not apply for Atlas platform. Consequently, a kinematic controller was designed as a preliminary solution for the Atlas [2]. This controller takes the difference between the desired orientation and the measured orientation (in unit quaternion) as the error and guarantees bounded error with an adjustable upper bound. However, even [2] showed errors and delays, therefore, improvements such as gain scheduling on [2] are needed to achieve better accuracy.

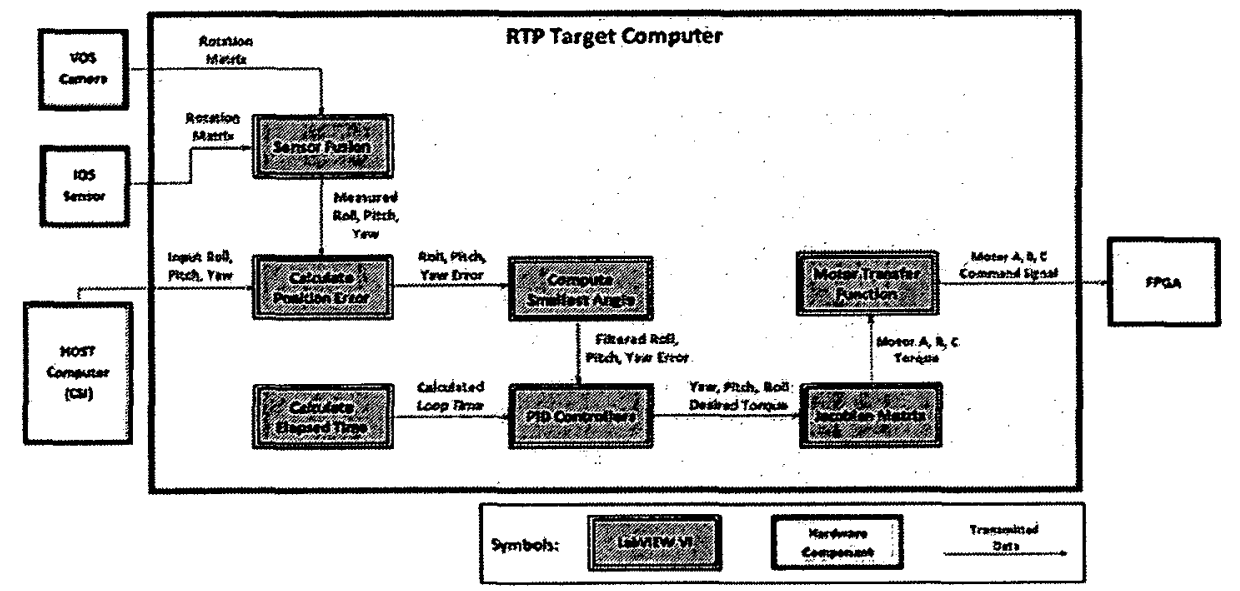

Figure 1.11: The PID control Sturcture for Atlas TDP.

\subsubsection{Sensor Fusion}

It is possible for the VOS to lose some accuracy when the sphere spins more rapidly as the camera is designed for stationary images. Conversely, the IOS will not lose any accuracy in dynamic motions but it is prone to drift and external disturbance [25]. Therefore, it is desired to fuse the VOS and IOS measurements to attain a more reliable measurement than each individual sensor. 
A simple application of a purely vision-based estimation using the extended Kalman filter (EKF) was designed and it involves the estimation of the pixel location on the image [26]. In terms of the VOS data filtering, this estimator is effective and easy to implement. However, it did not fuse the orientation measurement with another sensor (IOS in Atlas TDP). In addition, EKF is difficult to tune and only reliable for the systems that are almost linear on the time scale of the updates, it also needs a dynamic model to conduct the linearization. The unscented transformation (UT) was designed to propagate mean and covarance information through nonlinear transformation for the purpose of estimation [27] which led to the unscented Kalman filter (UKF). [28] provided a method of intergrating the triaxial gyscope data to quaternion estimation using UKF for the real-time estimation of a rigid body orientation. The UKF algorithm from [27] and [28] was used to fuse the IOS orientation and the VOS measurement orientation to get the estimated quaternion orientation on Atlas Lite [29]. This fusion algorithm was only tested in simulation and the results showed the error between the fused and real data was bounded in a small range while the IOS error increased over time. However, this UKF was never tested with the controller and sensors on a realtime system. In this research, a sensor fusion algorithm has to be designed based on UKF to fuse the VOS and IOS data on Atlas TDP.

\subsubsection{Quaternions}

The Atlas Simulator orientation is represented in a four-element quaternion which has the ability of representing any $3 \mathrm{D}$ orientation, as first introduced by Sir Rowan Hamilton in 1853 [30]. A quaternion is usually defined as a complex number as

$$
q=q_{0}+q_{1} i+q_{2} j+q_{3} k
$$


where $\left(q_{0}, q_{1}, q_{2}, q_{3}\right) \in R ; i, j$, and $k$ are three orthogonal unit spatial vectors. The quaternion is used for representing a rotation in Atlas as a unit quaternion which satisfies the normalization constraint given by

$$
q^{T} q=1
$$

where

$$
i^{2}=j^{2}=k^{2}=i j k=-1
$$

The quaternion multiplication between $q_{a}$ and $q_{b}$ is defined as

$$
q_{a} \cdot q_{b}=\left[\begin{array}{cccc}
q_{a 0} & -q_{a 1} & -q_{a 2} & -q_{a 3} \\
q_{a 1} & q_{a 0} & -q_{a 3} & q_{a 2} \\
q_{a 2} & q_{a 3} & q_{a 0} & -q_{a 1} \\
q_{a 3} & -q_{a 2} & q_{a 1} & q_{a 0}
\end{array}\right]\left[\begin{array}{c}
q_{b 0} \\
q_{b 1} \\
q_{b 2} \\
q_{b 3}
\end{array}\right]
$$

where $q_{a}=\left[\begin{array}{llll}q_{a 0} & q_{a 1} & q_{a 2} & q_{a 3}\end{array}\right]^{T} ; q_{b}=\left[\begin{array}{llll}q_{b 0} & q_{b 1} & q_{b 2} & q_{b 3}\end{array}\right]^{T}$. It is important to note that $q_{a} \cdot q_{b} \neq q_{b} \cdot q_{a}$. The quaternion has several benefits over Euler angles or axis angles including:

1. No singularities occur; and

2. Trigonometric functions are not used extensively in the mathematics (thereby favourably in influencing computation time).

So quaternions are selected as the representing format of orienation [31] in this research. 


\subsection{Objectives}

As described in the above sections, the Atlas TDP is incomplete in terms of the sensing system and the selected nonlinear controller. Since the TDP is a foundamental testing platform for the full-scale Atlas, it is necessary that every module including the actuation, system and simulation of TDP is designed, implemented and tested. At this stage of TDP, the acutation is completed and tesed. The TDP sphere is able to rotate around any of the three axes (roll, pitch, yaw) at a prescribed angular velocity based on the motor control and the Jacobian. It has been proven that the kinematic model and motor control performed as expected. However, no reliable dynamic model was ever developed for the TDP. A lot of work has been done on the simulation but they cannot be tested until the feedback loop is developed and the orientation of the sphere is controllable. The orientation sensing system is the prerequisite to completing the feedback orientation control of the Atlas TDP and it includes the existing IOS and a VOS to be designed and developed. Although a nonlinear quaternion-based kinematic controller was designed and tested on the Atlas Lite, improvements are necessary for the application of the controller on Atlas TDP. It is desirable to have a multi-sensor system to provide fast and accurate orientation data to the controller. Accordingly, a fusion algorithm of fusing the IOS and VOS is to be developed to contribute to the continuity and stability of the data flaw. Based on the statement above, the objectives of the research are summarized as follows:

1. Design a VOS system to measure the absolute orientation of the TDP sphere in real time. This sensing system will include the markers for positioning, lighting system to maintain the quality of the images, and a computing algorithm to calculate the orientation of the sphere from the locations of markers. It has to be robust and not sensitive to the changes in the environment. It should also be compuatationally efficient as it is part of a real-time system. 
2. Implement a robust nonlinear quaternion-based controller method to control the orientation of the sphere so that it can follow the desired trajectory. This controller has to be model-independent as there is currently no dynamic model for the TDP system.

3. Implement the UKF on the fusion of VOS and IOS data streams. It has to combine the advantages from both of the VOS and IOS to produce good quality output data with a high frequency and reliability.

\subsection{Contribution}

In this thesis, the following contributions are claimed:

1. Developed and tested a novel digital-ID marker system for the VOS system on Atlas TDP, which is robust to blur and environment lighting;

2. Implemented the sensor in the LABVIEW real-time environment and validated the performance of the the vision-based sensor;

3. Implemented a real-time nonlinear feedback controller together with the VOS system, which resulted in accurate dynamic trajectory following suitable for flight simulation; and

4. Applied the sensor fusion methods to fuse the VOS and IOS sensor outputs to create a more reliable output.

\subsection{Outline}

This section provides an overview of the following chapters in this thesis.

Chapter 2: Visual Orientation Sensor introduces the design concept of the visual 
orientation sensor as well as the preparation of the hardware construction and the real time testing of the software.

Chapter 3: Vision-based Orientation Control outlines the quaternion-based nonlinear controller with the vision-based feedback design. The implementation and realtime testing of this controller on the Atlas TDP are also discussed.

Chapter 4: Preliminary sensor fusion of VOS and IOS outlines the sensor fusion alogrithm of the VOS and IOS. The unscented Kalman filter is introduced and implemented as the fusion method. The performance of the fusion is displayed and analyzed.

Chapter 5: Conclusion highlights the results and conclusions from each of the main aspects of the thesis and proposes a few directions for future work. 


\section{Chapter 2}

\section{Visual Orientation Sensor}

In this chapter, a novel design of a visual orientation sensor (VOS) is first introduced, including the motivation for the design, background, and the design concept. The main elements in the development of this sensor are stated, such as the marker design and the camera calibration. It also covers the implementation of this sensor on TDP and the performance of the visual orientation sensor in real-time tests.

As described previously, the VOS is expected to produce reliable, accurate and absolute measurement of orientation to compensate for drift in the IOS data. The design requirements [15] of this novel sensor are:

1. Measure orientation of the sphere with an accuracy of $\pm 0.1^{\circ}$ in pitch, roll, and yaw;

2. Provide orientation data at a frequency such that IOS drift error does not exceed the sphere accuracy requirement; and

3. Measure orientation without any previous information about the sphere's motion (time independent);

Based on the above requirements, a vision-based sensor is considered a good option. The proposed VOS is designed to be based on an industrial digital camera, a lighting 
system, and unique markers attached to the sphere. The operating principles of the VOS are:

1. Attach distinct markers to preselected locations on the sphere surface;

2. Use a digital camera and lighting system located beside the sphere to take pictures of one portion of the sphere surface (Figure 2.1);

3. Measure the coordinates of all the markers denoted by $P_{s}$ in the sphere frame when the sphere is in its reference position;

4. Take images when the sphere is rotating, identify the visible markers and their identification numbers, and the coordinates $\left(P_{w}\right)$ of the markers in the global frame;

5. Calculate the rotation matrix $R_{\text {rot }}$ between the sphere frame and the global frame with the marker coordinates in both frames as in Equation (2.1),

$$
P_{w}=R_{\text {rot }} P_{s}
$$

and

6. Calculate the orientation of the sphere from the rotation matrix.

In this chapter, the first section introduces the purpose of the marker design, the design requirements, the testing method, and the final design of the markers. The second section describes the process of camera calibration. The third section discusses the implementation and testing of the VOS on the real-time platform (RTP). Finally, an evaluation is made of the performance of the entire system. 


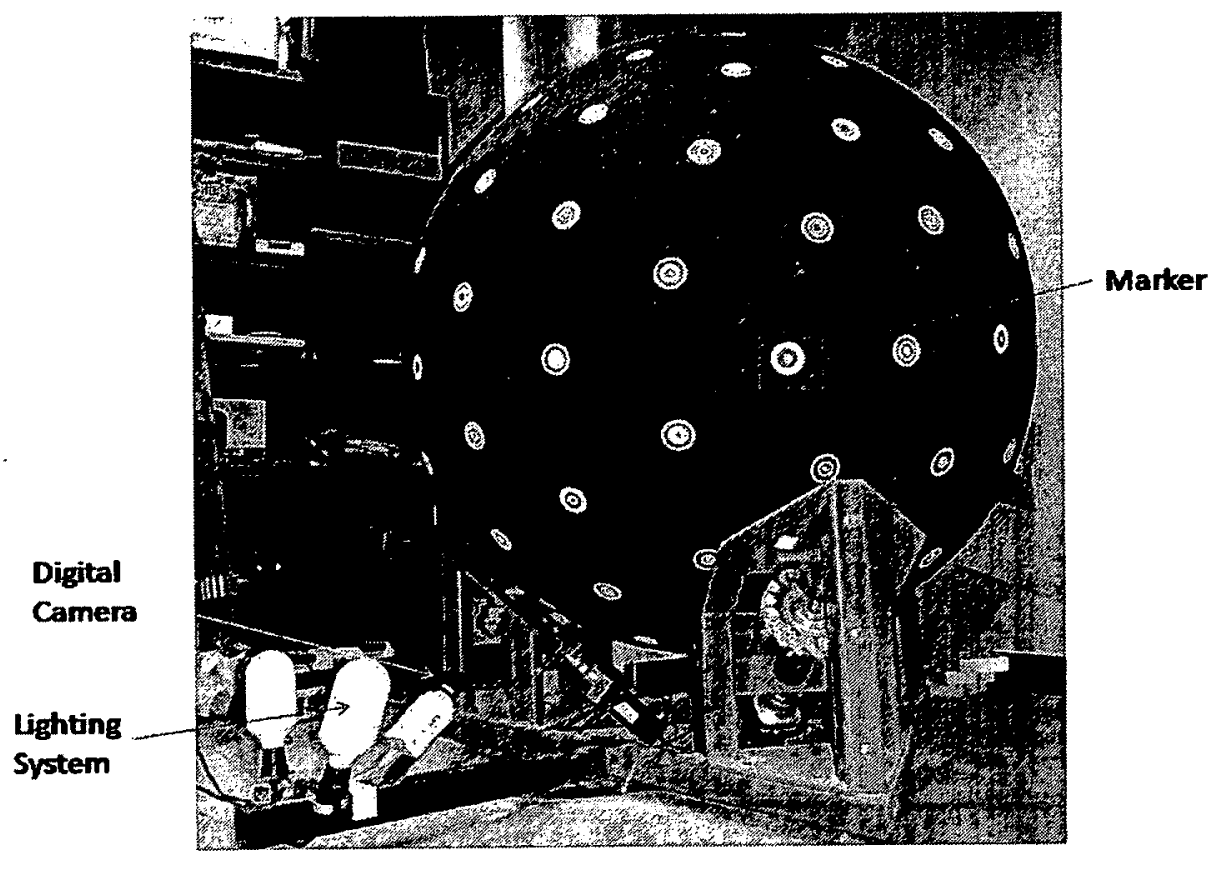

Figure 2.1: The marker system layout. 


\subsection{Marker Design and Testing}

\subsubsection{Introduction}

The orientation measurement of $3 \mathrm{D}$ rigid body needs markers as characteristic points. These markers need to be easily detected by the camera. Consequently, the design of the markers is crucial to the entire machine vision system. To meet the requirements of different applications, markers vary in their type, shape, and operating principles. In order to implement the VOS, a set of unique markers had to be developed and distributed evenly on the surface of the sphere. Several marker designs were considered, such as the single-colour marker im Figure 2.2, the mixed-colour markers in Figure 2.3, the Fiducial Artag markers in Figure 2.4, and finally the binary code markers in Figure 2.5. Following is a list of important properties for the markers used in this particular application:

1. The marker size should be large enough in the image, but small enough to prevent unnecessary distortion and calculation;

2. The use of colours should be minimal to avoid extensive computation;

3. The pattern should be centrosymmetric in order to meet the requirements of the rotation about roll, pitch, and yaw;

4. The pattern and the material of the markers should be robust and not sensitive to the simulator environment including the lighting conditions, dust, or minor physical damage;

5. The marker type should allow for a large number of distinct markers to permit adequate marker density on the sphere surface;

6. Together with the image processing process, the system should be capable of running in real time. 


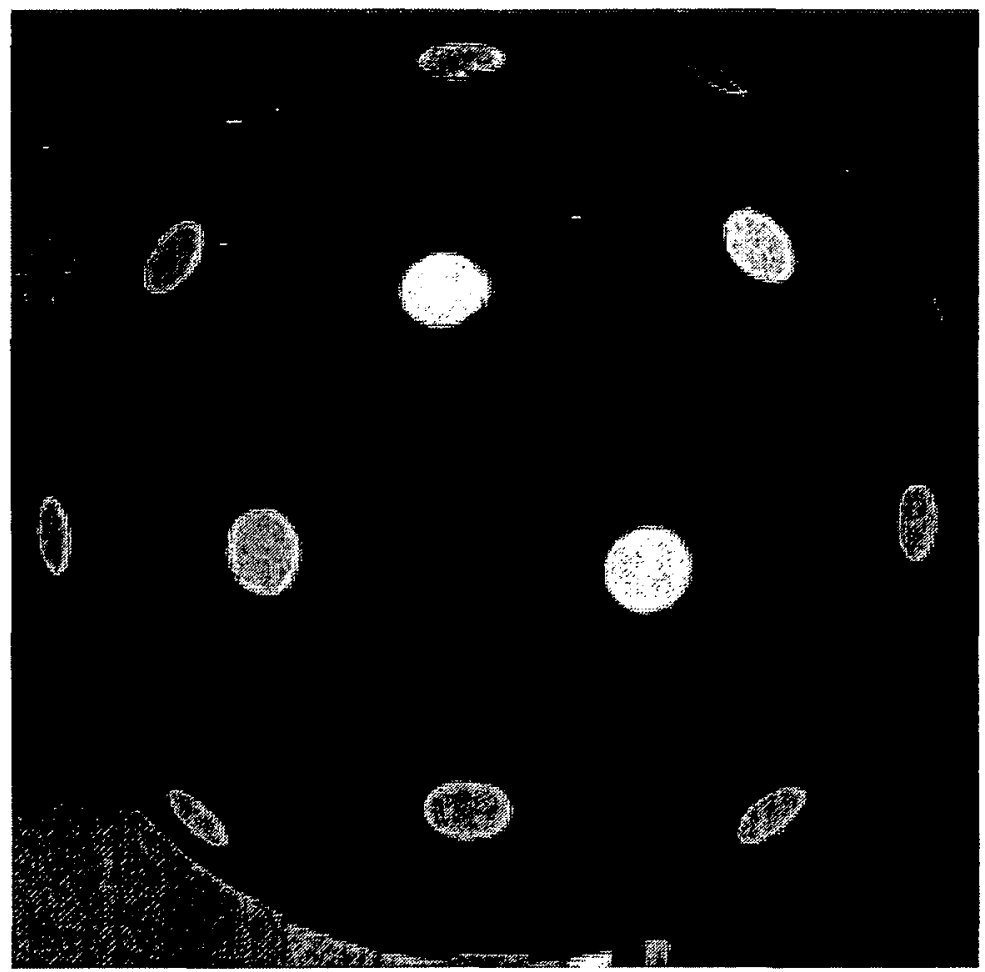

Figure 2.2: single colour markers on the desktop model.

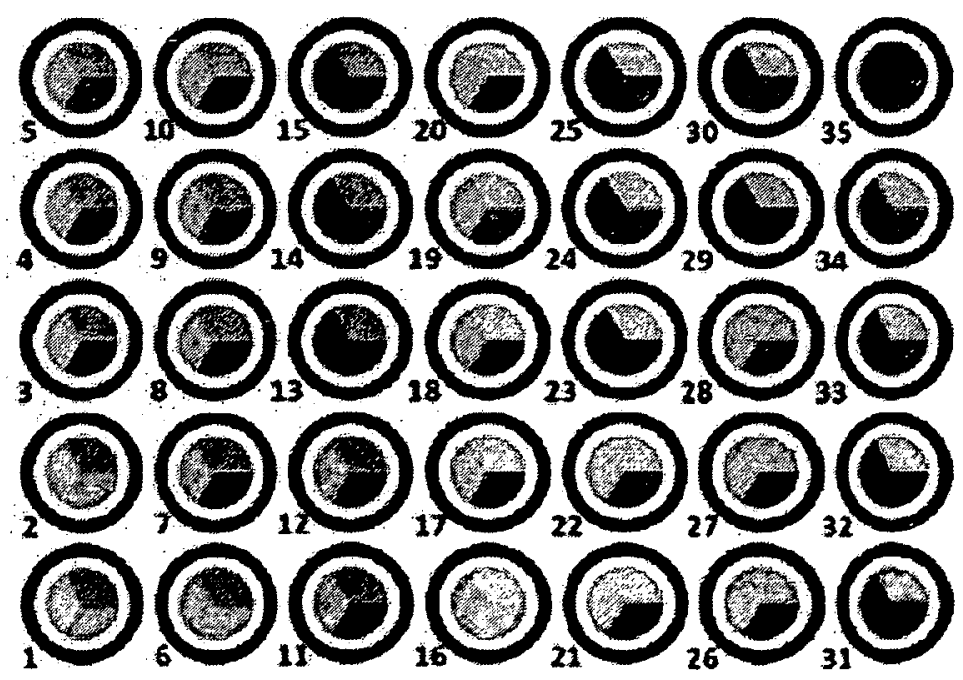

Figure 2.3: The mixed colour marker design. 


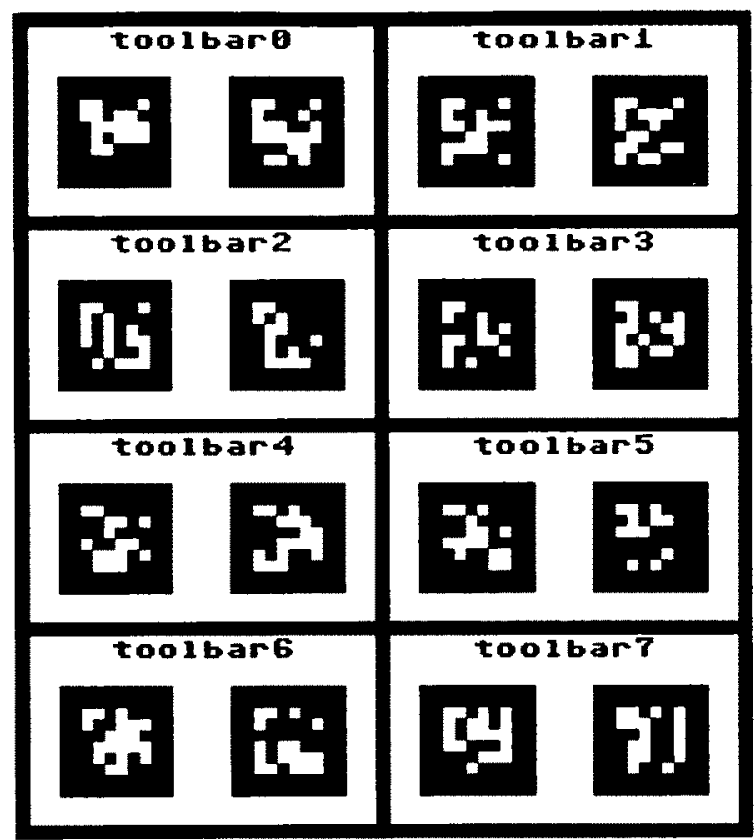

Figure 2.4: The ARtag markers.

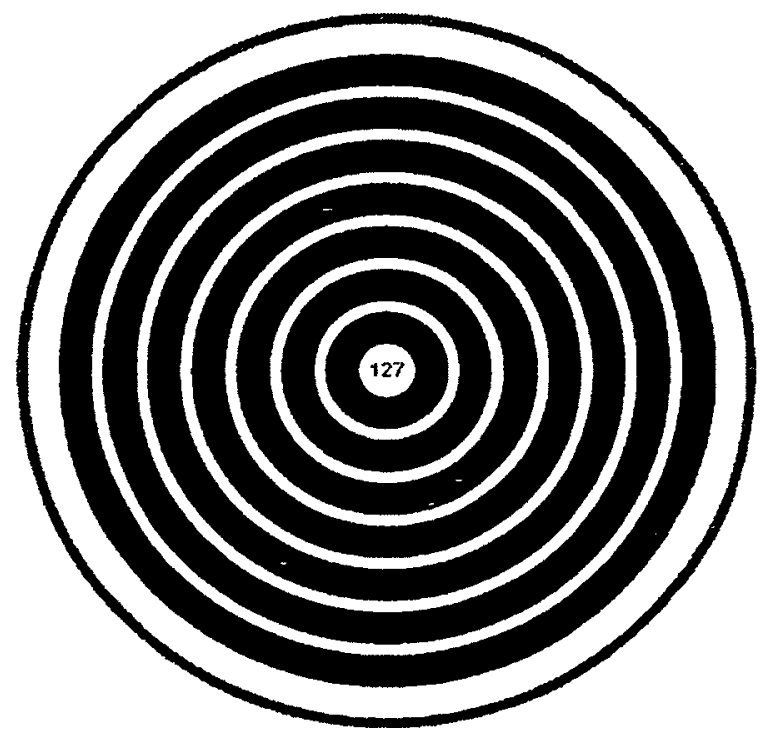

Figure 2.5: The design of number 127 binary marker. 
To get a sufficient number for the markers for identification in the orientation calculation of VOS, those markers have to be designed to contain unique features that are simple to detect and also maintain the clarity of the image. Several tests were carried out before finalizing the marker design to compare the performances of different marker designs.

\subsubsection{Original VOS Design}

As described in Section 2.1.1, three marker designs were tested on different Atlas models. In early stage of the development with Atlas Lite, ARtag markers were first considered because of their popularity in the field of Augmented Reality (AR). These markers are usually called fiducial markers and their false detection rate is quite low. Another benefit of these markers type is that it has a low misidentification rate between markers. While these features make ARtag markers a robust and reliable design [32]. However, this marker design contains more information than required, which introduces an unnecessarily large computational burden. As there is high pressure applied on the markers between the mecanum wheels and the sphere, the markers could be damaged, thus a center symmetrical pattern can maintain the information of the markers even they are damaged. Therefore, a custom sensing system, capable of real-time performance is preferred.

The single-colour markers shown in Figure 2.2 were proposed for the Atlas Lite model [15]. They are simple, easily constructed, and easily detected but have an obvious drawback. If more than ten colours are used, these colours would be very close in term of their characteristic frequency spectrum which would lead to a high potential exist for misidentification, particularly when lighting conditions are poor. Therefore, this concept was not favoured for the VOS design. Instead of the single-colour markers, mixed-colour markers were also designed as shown in Figure 2.3 [33]. The mixed-colour 
design provides more markers than the single colour markers without requiring any new colour because they combine three colours into one marker. This solution was satisfactory for Atlas Lite with the need of 32 markers. However, as the project moved onto the Atlas TDP, the number of the markers increased to 122. The mixed-colour scheme can hardly provide a sufficient number of strongly-distinguishable markers which led to similar issues. Furthermore, the colours may fade as the sphere continues to run in contact with the wheels. The colour marker identification is also very sensitive to the lighting conditions, thus introducing stringent requirements on the marker identification hardware.

In order to avoid using colours, a binary code marker which is made of several black or white concentric circles with various diameters and thicknesses was proposed and preliminary tests were conducted [18]. A typical marker is shown in Figure 2.5. Each ring or circle on the markers represents the binary digit 1 or 0 , so each of them represents a single binary number. Four centimeters diameter binary code markers were used on the Atlas TDP. The widths of all the rings are $2 \mathrm{~mm}$ while the empty sections between the rings are $0.5 \mathrm{~mm}$ in width. This pattern caused a lot of blurs in the dynamic images taken when the sphere is rotating. This blur reduces the quality of the images and leads to a low detection rate. Despite this, compared the colour markers, binary code markers have several advantages including:

1. The binary markers are able to provide a greater number of markers than the colour markers;

2. The binary markers are black and white in colour, so that a black and white camera is sufficient;

3. The black and white rings on the markers are less sensitive to the lighting conditions compared to colours markers; 


\subsubsection{Final Design: Binary Code Markers}

After being compared with competing marker designs, the binary code markers were selected as the final marker choice. To compute an accurate rotation matrix, $R_{\text {rot }}$, the images of the sphere have to contain at least two good-quality markers for identification. Usually one additional marker is taken into account for improving the accuracy of the rotation matrix. Therefore, an average of three markers should appear in the central area of an image whatever motion of the sphere. Three markers identified in the central part would lead to five or six markers in the entire image as the markers at the edges of the images are strongly distorted and are not taken into consideration and the calculation process. The projection area of the sphere is about $2000 \mathrm{~cm}^{2}$. Since the simulation work is carried out on a mid-scale model whose radius is $62 \mathrm{~cm}$ and the surface area of the sphere is $48280 \mathrm{~cm}^{2}$. So we can find out there are approximately 24 similar areas all around the sphere with five markers per area. This results in 120 markers required to fully cover the entire sphere. Figure 2.6 shows the procedure of deciding the marker numbers. It was ultimately found that due to practical constraints 122 markers are needed.

To produce 122 different binary numbers, the markers are designed to contain seven rings $\left(2^{7}=128\right)$. Details of the binary marker design had to be determined such as the diaster of the marker, the thickness of the rings, and the distance between the rings. Thus, an experiment was performed to find out the best features of the markers. In the tests, three markers were pasted on two different background colour testing boards as shown in Figure 2.7. The markers were put into several groups and they were all attached to the boards during the test. 


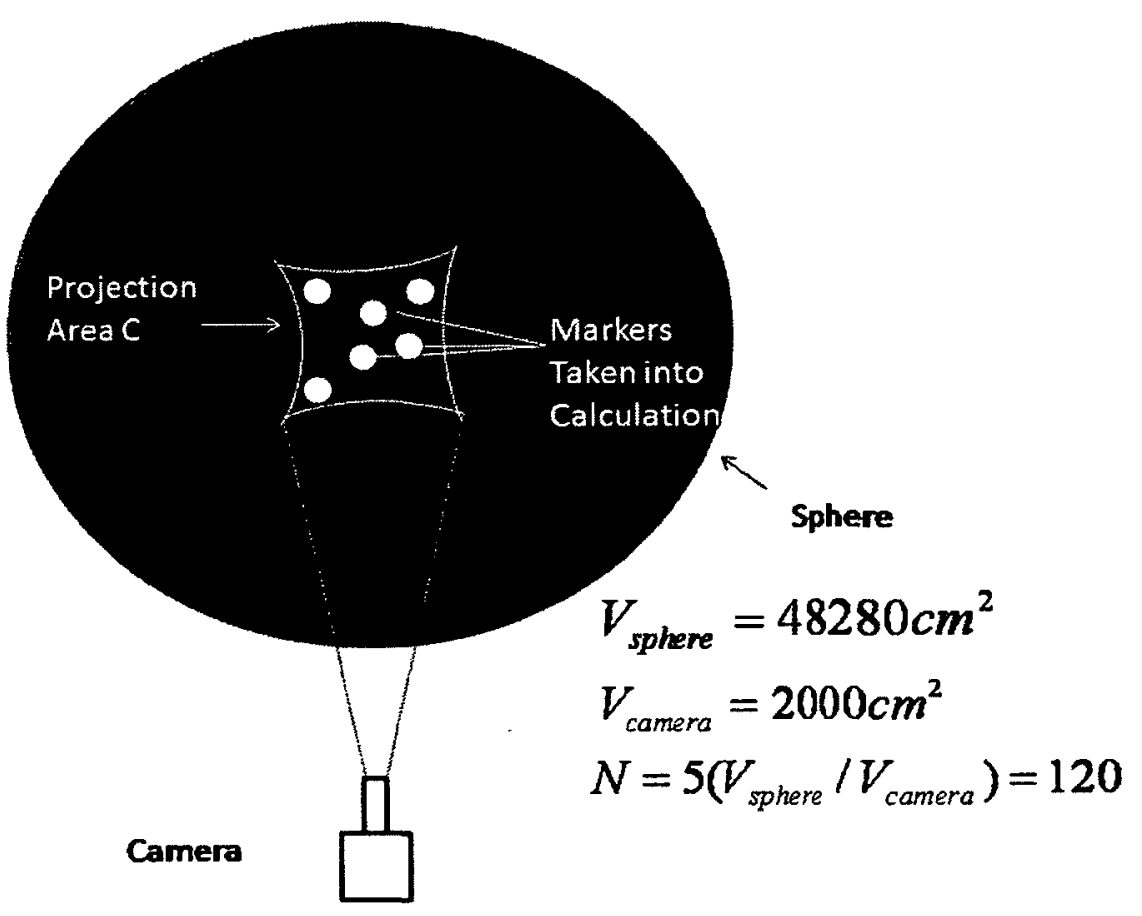

Figure 2.6: Diagram illustrating calculation of the marker number. 


\section{Marker Design Test on CRS Robot}

To generate an independently measurable rotation having the same linear velocity at the end effector as expected to the surface of the TDP sphere, a CRS robot manipulator shown in Figure 2.8 was used. During the tests, the boards were fixed to the end effector of the manipulator while the manipulator was rotating around its first joint at a certain angular speed. Since the length from the first joint to the end effector was $71 \mathrm{~cm}$ (and the radius of the TDP was $62 \mathrm{~cm}$ ), the required angular velocity of the CRS robot was calculated as:

$$
\omega_{c r s}=\left(r \omega_{\text {sphere }}\right) / l
$$

where $\omega_{\text {crs }}$ is the angular velocity of the robot arm; $\omega_{\text {sphere }}$ is the maximum angular velocity of the sphere; $r$ is the radius of the sphere and $l$ is the length of the robot arm. Since $\omega_{\text {sphere }}=35$ degrees $/$ second, $\omega_{\text {crs }}=30.5$ degrees $/$ second.

The camera was arranged next to the robot manipulator and took pictures of the testing boards during the rotation of the robot manipulator. Sample pictures taken are shown in figure 2.7.

The results from the tests are shown in Table 2.1 (All the images can be seen in the Appendix A), from which several principles of the marker design were concluded as follows.

1. Compared with the $4 \mathrm{~cm}$ diameter markers, those with a diameter of $6 \mathrm{~cm}$ provide better image quality, with less blur and stronger contrast.

2. Compared with the markers whose ring width is $0.5 \mathrm{~mm}$, those markers with 1 $\mathrm{mm}$ wide rings provide better image quality with less blur. 


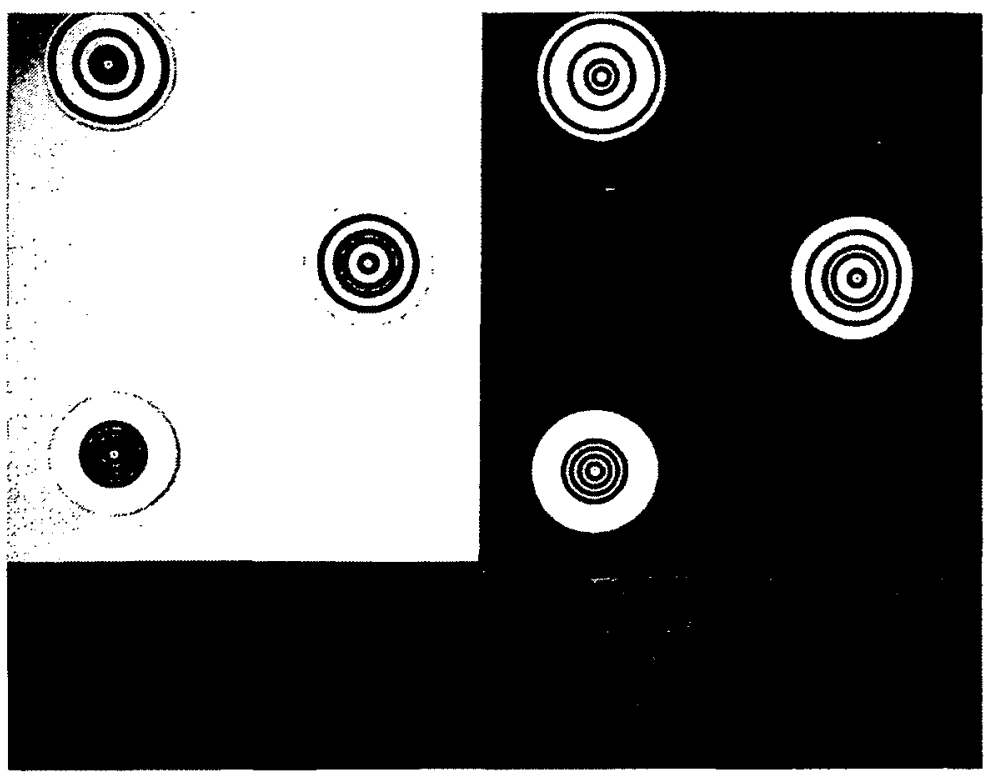

Figure 2.7: Marker boards for marker design testing.

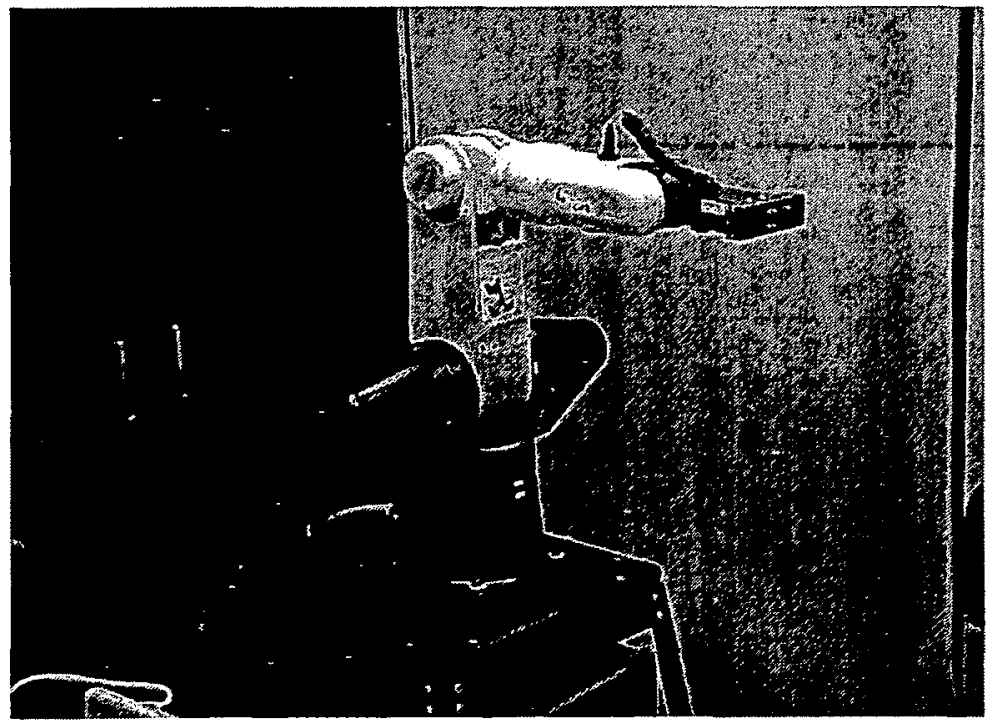

Figure 2.8: CRS manipulator. 
Table 2.1: The test results of binary marker design.

\begin{tabular}{|c|c|c|c|c|}
\hline Number & $\begin{array}{l}\text { Background } \\
\text { Colour }\end{array}$ & $\begin{array}{l}\text { Marker } \\
\text { Diameter } \\
(\mathrm{cm})\end{array}$ & $\begin{array}{l}\text { Ring } \\
\text { Width } \\
(\mathrm{mm})\end{array}$ & Image Quality \\
\hline 1 & Black & 6 & 0.5 & $\begin{array}{l}\text { good contrast, } \\
\text { normal blur }\end{array}$ \\
\hline 2 & Black & 4 & 0.5 & $\begin{array}{l}\text { good contrast, } \\
\text { strong blur }\end{array}$ \\
\hline 3 & Black & 6 & 1 & $\begin{array}{l}\text { good contrast, } \\
\text { less blur }\end{array}$ \\
\hline 4 & Black & 4 & 1 & $\begin{array}{l}\text { good contrast, } \\
\text { more blur }\end{array}$ \\
\hline 5 & White & 6 & 0.5 & $\begin{array}{l}\text { too bright, more } \\
\text { blur }\end{array}$ \\
\hline 6 & White & 4 & 0.5 & $\begin{array}{l}\text { too bright, nor- } \\
\text { mal blur }\end{array}$ \\
\hline 7 & White & 6 & 1 & $\begin{array}{l}\text { too bright, less } \\
\text { blur }\end{array}$ \\
\hline 8 & White & 4 & 1 & $\begin{array}{l}\text { too bright, more } \\
\text { blur }\end{array}$ \\
\hline
\end{tabular}


3. Compared with the white background, the black background caused less blur in the image.

With above observations, the final design of the binary markers involves:

1. Changing the diameter of the markers from $4 \mathrm{~cm}$ into $6 \mathrm{~cm}$.

2. Increasing the width of the empty sections by $0.5 \mathrm{~mm}$.

3. Using a black background.

The 122 markers were then evenly distributed on the surface of sphere. Their coordinates on the surface of the sphere was calculated and then Optotrak was used to locate the markers. The markers with close IDs (such as $7,8,9$ ) were put together for the purpose of error checking. If a detected marker ID is not close to adjacent markers's IDs, this marker will then be determined as a misidentified marker.

\subsubsection{Obtaining the Marker Positions in the Sphere Frame}

In order to identify the sphere orientation through the rotation matrix $R_{\text {rot }}$, the position of the centre of each marker denoted as $P_{s}$ must be measured with respect to the local sphere frame. To measure the $P_{s}$ as precisely as possible, a Optotrak [34] Motion Capture System was used. It has an accuracy of $0.1 \mathrm{~mm}$ and resolution of $0.01 \mathrm{~mm}$ in 3D space. The measurement system and Optotrak are shown in Figure 2.9 and 2.10 .

Every marker on the sphere was measured by the Optotrak to get its coordinates in the sphere frame. The Optotrak is set up three metres from the sphere. Since the markers are all over the sphere, only $30 \%$ of the markers can be measured in the first round and their coordinates are denoted as $P_{s 1}$. To measure the rest of the 


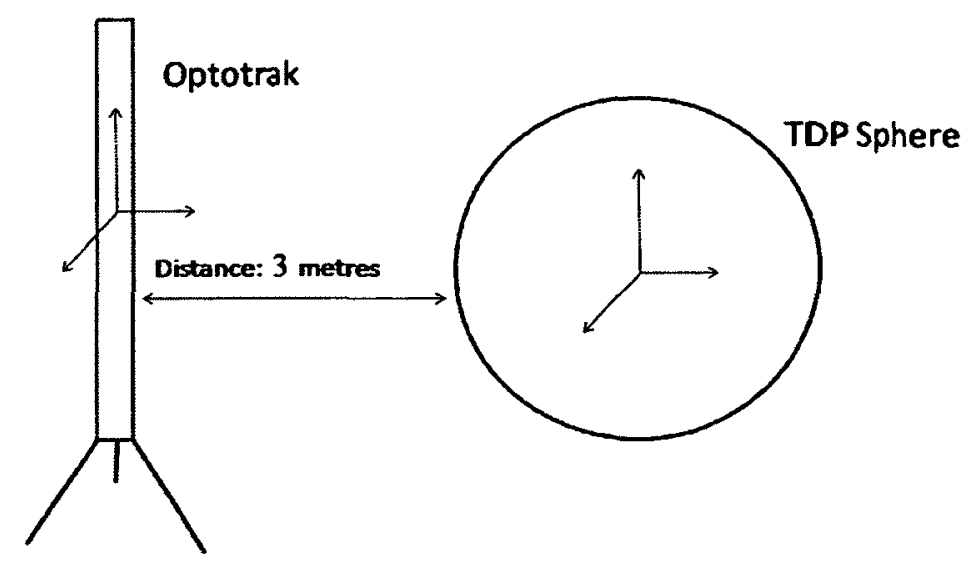

Figure 2.9: The Optotrak measurement system.

markers, the sphere had to be rotated so that the left markers are facing the Optotrak. However, in the second round, the marker locations were measured in a different frame $F_{2}$ other than the original Sphere Frame $F_{1}$. Thus, a transformation matrix $H$ had to be applied to the coordinates of the markers denoted as $P_{f 2}$. Theoretically, simple rotation matrix should be able to represent the transformation from the sphere frame to the rotated frame. However in the case of the TDP, the sphere is not perfect and rotation on the mecanum wheels cannot guarantee that rotation is exactly about the geometric centre of the sphere. In other words, the transformation had to be treated as a rotation plus translation. In order to get the homogeneous transformation matrix between $F_{1}$ and $F_{2}$, four reference markers were selected so that $H$ was obtained from the two $4 \times 4$ position matrices of the reference markers. They are measured in both frames. The sphere frame coordinates of the rest of the markers in the second round of measurement were computed as : $P_{s 2}=H P_{f 2}$. The same method is applied until every marker's coordinates in the sphere frame were measured. A schematic diagram of the entire process is provided in Figure 2.11. The UltrCoordinates.txt document that contains the coordinates of all the markers in the sphere frame is included in Appendix B. From the tests on the precision of the marker positions, it was found 
that the errors between the real positions and calculated positions are less than $5 \mathrm{~mm}$.

\subsection{Camera Calibration}

\subsubsection{Introduction}

Camera calibration is an important process in machine vision, especially in the threedimensional applications. According to Roger Tsai's definition [35], camera calibration in the context of three dimensional (3D) machine vision is the process of determining the internal camera geometric and optical characteristics (intrinsic parameters) and the $3 \mathrm{D}$ position and orientation of the camera frame relative to a certain global coordinate system (extrinsic parameters). The intrinsic parameters of the camera usually contain the focal length in both directions $f_{x}$ and $f_{y}$, the centre of the camera frame $C_{x}$ and $C_{y}$ and the distortions $k$ of the lens. The extrinsic parameters are the translation matrix and rotation matrix between the camera frame and the global frame. As shown in Figure 2.12, a point $\mathrm{P}$ in the world frame has two groups of coordinates in the global frame and camera frame respectively; $Q$ is representing the extrinsic parameters which include the translation matrix $\left[T_{x}, T_{y}, T_{z}\right]^{T}$ and rotation matrices around yaw, pitch, and roll axes; $R_{\text {camera }}$ represents the camera frame and $R_{\text {world }}$ represents the world frame; and $\left.P\right|_{\text {camera }}$ and $\left.P\right|_{\text {world }}$ are the coordinates of $P$ in both the camera and world frames respectively. The purpose of the camera calibration is to map the transformation between the image frame and the global frame. In other words, the objective of camera calibration is to reach the point whereby if the image processing program gets the coordinates of the marker in the image frame, the corresponding coordinates of the marker in the global frame can be found. The process of camera 


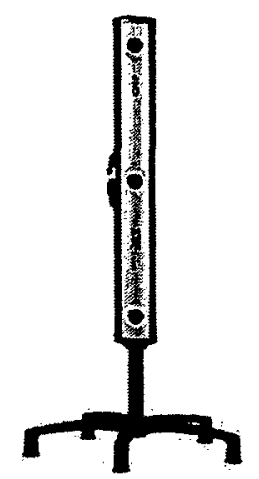

Figure 2.10: Position sensor Optotrak.

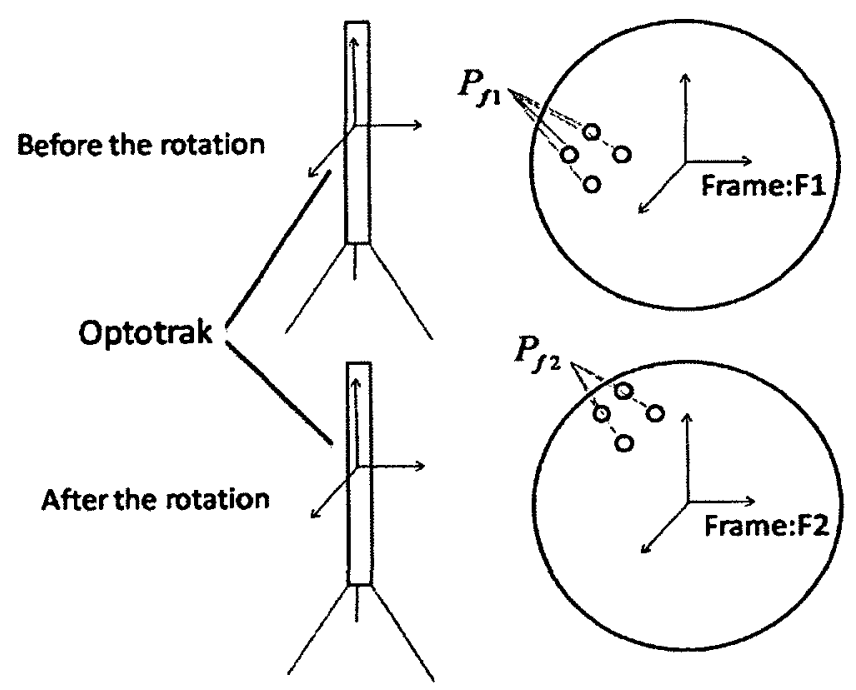

Figure 2.11: Demonstration of the process of measuring $P_{s}$. 


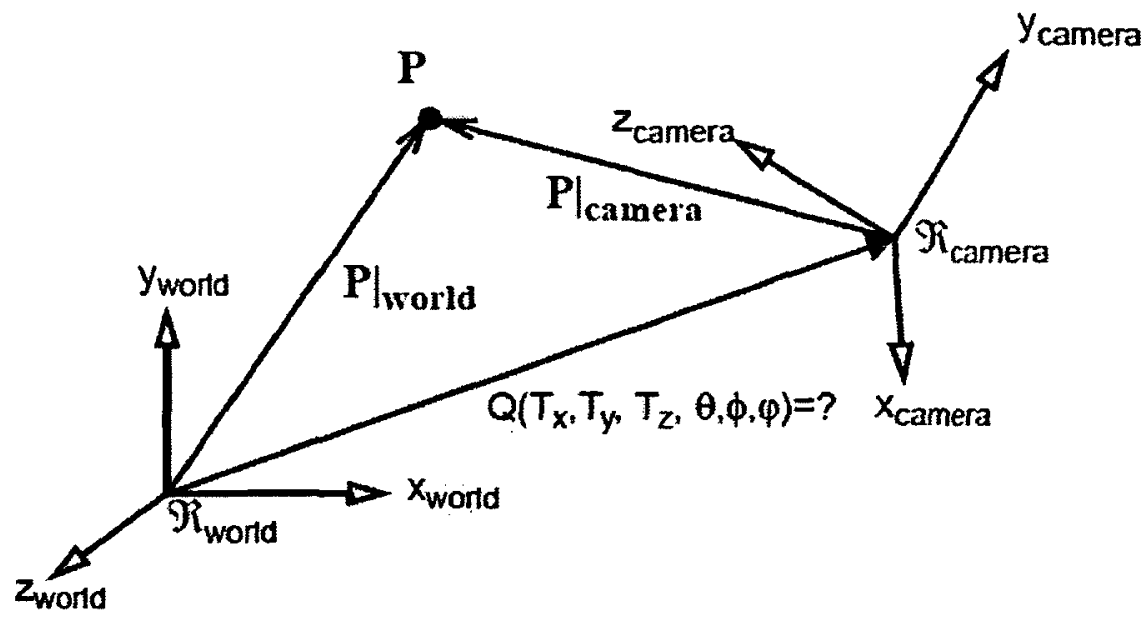

Figure 2.12: The transformation between camera frame and global frame [1].

calibration in this case can be summarized as getting the intrinsic parameters first and then do a set of experiments using the Optotrak to get the coordinates of several points in both of the sphere frame and the image frame to calculate the extrinsic parameters. Subsections 2.2.2 and 2.2.4 introduce the two main processes of the camera calibration: obtaining the intrinsic parameters and obtaining the extrinsic parameters.

\subsubsection{Intrinsic Parameters}

\section{Camera Model}

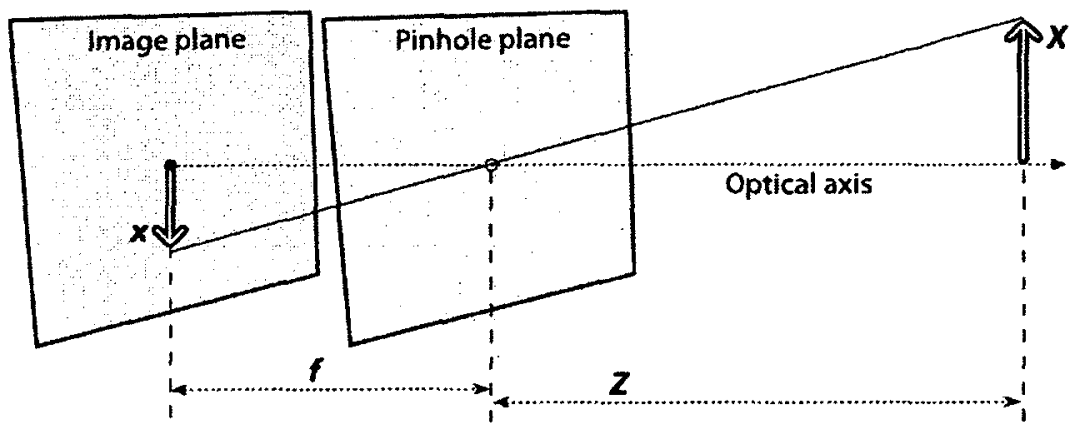

Figure 2.13: A pinhole camera model. 
This subsection describes how to get the intrinsic parameters of the camera in the context of Atlas. The very first step is to understand the camera model. The camera calibration problem is defined using a simple camera model, usually a pinhole model, as shown in Figure 2.13 [1]. In this pinhole model, the point in the global frame at the position of $[X, Y]^{T}$ is projected to $[x, y]^{T}$ in the image plane through the pinhole. The parameter $f$ is the focal length of the lens, $Z$ is the distance between the object and the pinhole plane (lens), $x$ and $y$ are the coordinates in the image plane, and $X$, $Y$ are the corresponding coordinates of the point in the global frame. The projection function is

$$
x=f \frac{X}{Z}
$$

Normally, in an image frame, the origin of the coordinate system is put at the upper left corner of the image. In this case, the projection function between the coordinates of an object in the global frame and the coordinates in the image frame will be

$$
\begin{aligned}
& x_{i m g}=f_{x}\left(\frac{X}{Z}\right)+C_{x}, \\
& y_{i m g}=f_{y}\left(\frac{Y}{Z}\right)+C_{y},
\end{aligned}
$$

where $C_{x}$ and $C_{y}$ are the coordinates of the centre of the sphere in the image coordinate system. In Tsai's paper [35], $\mathrm{X}$ is the global coordinate, the $x_{i m g}$ and $y_{i m g}$ are the coordinates in the image frame with the origin at the upper left corner. The centre of the image is not always the theoretical centre of the image plane, thus it needs to be calculated in the camera calibration process. With the definitions above, the 
transformation matrix between the global frame and the image frame can be found as,

$$
q=M Q
$$

where $q=\left[\begin{array}{c}x \\ y \\ w\end{array}\right], M=\left[\begin{array}{ccc}f_{x} & 0 & C_{x} \\ 0 & f_{y} & C_{y} \\ 0 & 0 & 1\end{array}\right], Q=\left[\begin{array}{c}X \\ Y \\ Z\end{array}\right]$ where $x, y$ are the coordinates of the point in the image frame, $w$ is a scalar, $X, Y$ and $Z$ are the global coordinates of the point, $f_{x}$ and $f_{y}$ are the focal length along the $x$ and $y$ axes in the image frame and $C_{x}$ and $C_{y}$ represent the distance between the centre of the image and the origin of the image frame [1].

The $Q$ in Equation (2.6) denotes the coordinates of the point in the global frame and $q$ denotes the projection of $Q$ in the image frame. Equation (2.6) can be reduced to

$$
\left[\begin{array}{c}
x \\
y \\
w
\end{array}\right]=\left[\begin{array}{c}
f_{x} X+C_{x} Z \\
f_{y} Y+C_{y} Z \\
Z
\end{array}\right]
$$

where $w=Z$, so if we divide the matrix on the right by $Z$, we can get the original coordinates of the projection point which are the same as in Equation 2.4 and 2.5. Note that here the negative sign is ignored since its function is to merely indicate the relative direction of the image. In Equation 2.6, the $3 \times 3$ matrix contains the "Intrinsic Parameters", since it contains the focal length of the camera and the centre of the image frame. 
In the above derivation, it is assumed the lens is in perfect condition, which means there is no distortion at all. However, at minimum small distortion always exists and affects the accuracy of the camera model. Depending on the camera quality, the distortion varies significantly. From the description in $[1,35]$, the most significant distortions are the radial and tangential distortions. In most approaches, two coefficients $k_{1}$ and $k_{2}$ (sometimes a third distortion coefficient $k_{3}$ ) are introduced to capture the effect of radial distortion as well. The correcting terms to compensate for the radial distortion at a point are expressed by [1]

$$
\begin{aligned}
& X_{\text {correct }}=X\left(1+k_{1} r^{2}+k_{2} r^{4}+k_{3} r^{6}\right), \\
& Y_{\text {correct }}=Y\left(1+k_{1} r^{2}+k_{2} r^{4}+k_{3} r^{6}\right)
\end{aligned}
$$

where $X, Y$ are the original coordinates; $X_{\text {correct }}$ and $Y_{\text {correct }}$ are the new coordinates after compensating; $k$ is the distortion coefficient; and $r$ is the radial distance from the centre of the lens to the point.

For the tangential distortion, the correcting equations are

$$
X_{\text {correct }}=X+\left[p_{1}\left(r^{2}+2 X^{2}\right)+2 p_{2} X\right]
$$

and

$$
Y_{\text {correct }}=Y+\left[p_{1}\left(r^{2}+2 Y^{2}\right)+2 p_{2} Y\right]
$$

the notations here are the same as for Equation 2.8 and 2.9. $p_{1}$ and $p_{2}$ are the coefficients of the tangential distortions. Therefore, the overall intrinsic parameters contains the focal length, the centre of the image frame, and the distortions: $P_{\text {camera }}=\left[f_{x}, f_{y}, C_{x}, C_{y}, k_{1}, k_{2}, k_{3}, p_{1}, p_{2}\right]^{T}$. To get the intrinsic parameters, both the OpenCV camera calibration functions and MATLAB camera calibration toolbox were used. The results of implementing the two approaches are presented in the following 
sections.

\subsubsection{Obtaining the Intrinsic Parameters}

The process of obtaining the intrinsic parameters was carried out using two packages to ensure accuracy: OpenCV and MATLAB. OpenCV is the Open Source Computer Vision Library in $\mathrm{C}$ and $\mathrm{C}++$. It has been widely used in machine vision since it provides many basic image processing functions and is focused on real-time vision. For Atlas, the final goal is to implement a real-time platform. Thus OpenCV was selected as the program for all the image capturing, image processing, and camera calibration. OpenCV follows the camera calibration method proposed in the paper " $A$ flexible new technique for camera calibration” by Z.Zhang [36]. With Zhang's method, a chessboard is needed as shown in Figure 2.14. The dimensions of the squares in the chessboard should be measured accurately. When the physical size of the squares of the chessboard and the size of the squares in the image are known, the intrinsic parameters could be determined. The reason to use the chessboard is the ease of processing of the black and white squares to obtain the pixel-measured distance between the corners. In some cases, researchers also use other features such as black circles on a white background with working principles the same as with the chessboard.

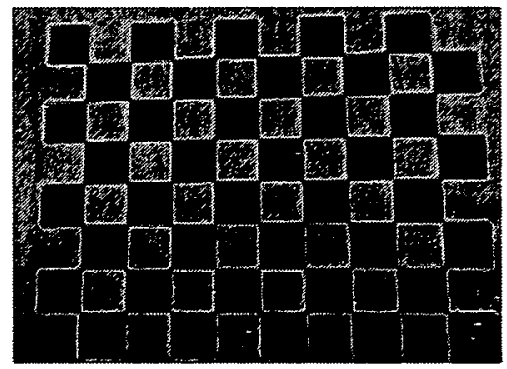

Figure 2.14: Chessboard for Camera Calibration.

In OpenCV, the "CalibrateCamera" function is specifically built for the calibration 
purpose. The descriptions are on the OpenCV official site [1]. A program is provided as well, which is able to calculate all the intrinsic parameters as well as the distortion coefficients if proper inputs are given. On the other hand, Jean-Yves Bouguet from the Computer Vision Research Group in the Department of Electrical Engineering at the California Institute of Technology developed the Camera Calibration Toolbox for MATLAB [37]. To get the intrinsic parameters, the MATLAB camera calibration toolbox loads the images of the chessboard first, and then asks the user to manually pick the four vertices of the chessboard. All the corners in the chessboard are then extracted. Similar to the OpenCV program, the real size of the squares on the chessboard must be provided to the MATLAB program as an input.

A large number of calibration images was generated by rotating the frame in $3 \mathrm{D}$ space.

Table 2.2: The Intrinsic Parameters from OPENCV and MATLAB

\begin{tabular}{|l|l|l|l|}
\hline Parameters & OPENCV Approach & MATALB Approach & Difference \\
\hline $\mathrm{f}_{x}$ & 745.3273 & 748.1803 & 2.853 \\
$\mathrm{f}_{y}$ & 745.9363 & 748.6416 & 2.7053 \\
$\mathrm{C}_{x}$ & 639.5 & 644.9848 & 5.4848 \\
$\mathrm{C}_{y}$ & 511.5 & 512.4329 & 0.9329 \\
$\mathrm{k}_{1}$ & -0.05628 & -0.05863 & -0.00163 \\
$\mathrm{k}_{2}$ & 0.1208 & 0.12103 & 0.00023 \\
$\mathrm{p}_{1}$ & 0.001559 & 0.0012 & -0.000359 \\
$\mathrm{p}_{2}$ & -0.0009557 & 0.00045 & 0.0014057 \\
\hline
\end{tabular}

In Table 2.2 we have 2 groups of the intrinsic parameters calculated from the OpenCV program and the MATLAB Camera Calibration Toolbox. The differences between the two groups of values are within $1 \%$ and have no noticeable influence on the extrinsic parameter calculation. 


\subsubsection{Extrinsic Parameters}

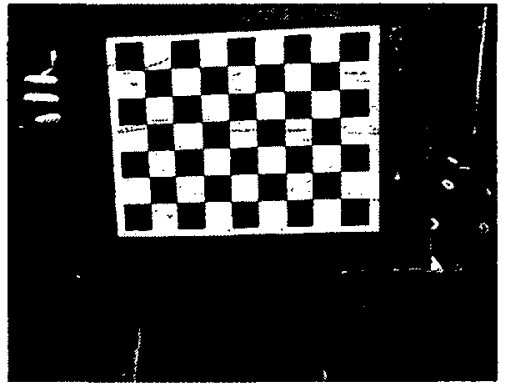

(a) Checkboard Position 1

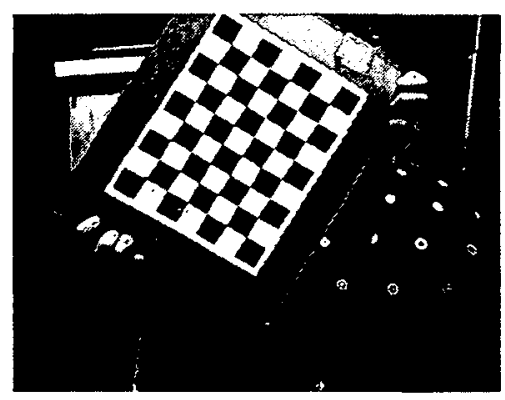

(b) Checkboard Position 2

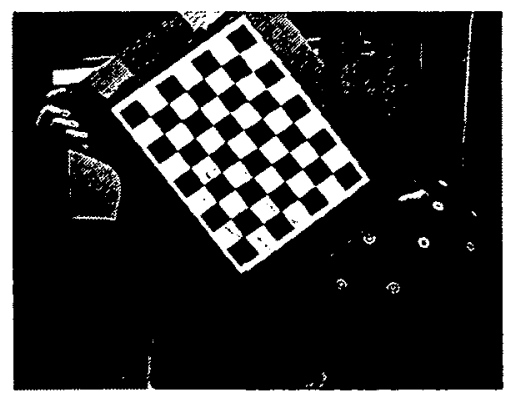

(c) Checkboard Position 3

Figure 2.15: Different positions of the check board

In this section, the extrinsic parameters are calculated based the intrinsic parameters. The extrinsic parameters describe how a point in the global frame is projected to the image frame. The projection relationship is represented by two matrices: a rotation matrix $H_{r}$ and a translation matrix $H_{t}$. The most common way to get the extrinsic parameters is to align the chessboard with the global frame, and then use multiple images such as in Figure 2.15 to calculate both the intrinsic parameters and extrinsic parameters. However, this method is not possible for the Atlas TDP, because the sphere blocks the centre of global frame from the camera. If the chessboard needs to be placed at the location of the global frame, a special mechanism would need to be designed to support the chessboard with the sphere not present. In the case of the Atlas TDP, however, there is no low cost way to find the chessboard's locations with high precision. Another method had to be proposed to obtain the extrinsic parameters. 
Instead of the experimental method using the chessboard, a mathematical method was developed to provide a very good and reliable approach to calculate the extrinsic parameters. Recalling the overall transformation function between the global frame and the image frame (Equation 2.6), if $q$ and $Q$ are set as homogeneous coordinates and redefined as $Q=\left[\begin{array}{llll}X & Y & Z & 1\end{array}\right]^{T}$ and $q=\left[\begin{array}{lll}x & y & 1\end{array}\right]^{T}$. Without loss of generality, the projection function between the world frame and the image frame can be converted into

$$
s \cdot\left[\begin{array}{l}
x \\
y \\
1
\end{array}\right]=\left[\begin{array}{ccc}
f_{x} & 0 & C_{x} \\
0 & f_{y} & C_{y} \\
0 & 0 & 1
\end{array}\right] \cdot\left[\begin{array}{llll}
r_{11} & r_{12} & r_{13} & r_{14} \\
r_{21} & r_{22} & r_{23} & r_{24} \\
r_{31} & r_{32} & r_{33} & r_{34}
\end{array}\right] \cdot\left[\begin{array}{c}
x \\
y \\
z \\
1
\end{array}\right]
$$

where $s$ is scalar coefficient; $r_{i j}$ are the elements of the homogeneous transformation matrix between the world frame and the image frame; $\left[\begin{array}{cccc}r_{11} & r_{12} & r_{13} \\ r_{21} & r_{22} & r_{23} \\ r_{31} & r_{32} & r_{33}\end{array}\right]$ is the rotation matrix $H_{r}$ of the extrinsic parameters and $\left[\begin{array}{lll}r_{14} & r_{24} & r_{34}\end{array}\right]^{T}$ is the translation matrix $H_{t}$ of the extrinsic parameters; $\left[\begin{array}{llll}x & y & z & 1\end{array}\right]^{T}$ and $\left[\begin{array}{lll}x & y & 1\end{array}\right]^{T}$ are the coordinates of the point in the global frame and the image frame respectively. Since there are four arguments in the equation, if three of them are known then the fourth one can be calculated. Therefore, the mathematical approach is to get the intrinsic parameters, the points coordinates in the image frame, and the corresponding coordinates in the global frame in advance, and then use OpenCV and MATLAB to calculate the unknown extrinsic parameters. The problem turns into how to get the corresponding coordinates in both the global and the image frames. 
Obtain the Coordinates in both the Global Frame and the Image Frame

The coordinates in the image frame are easy to generate using image processing techniques. Firstly, a pattern as shown in Figure 2.16 is designed and pasted on the sphere at ten different locations within the camera field of view. With this design, the centre $P$ of the four squares is very easy to detected using a corner detector with small error. The camera takes ten images when the pattern is at ten different locations, and then computes all the points $P$, named $P 1$ through $P 10$. Figure 2.17 shows the image for computing the coordinates in the image frame.

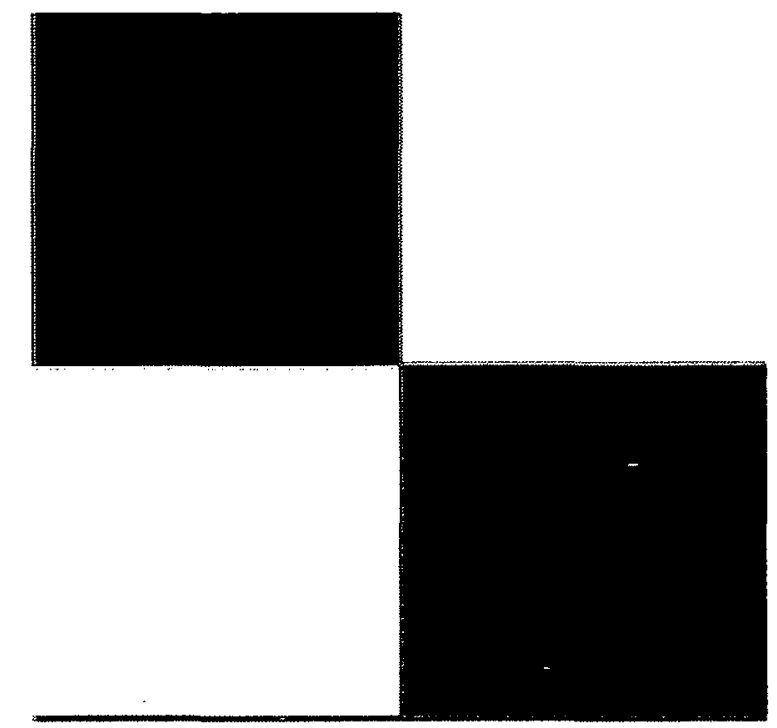

Figure 2.16: 4 squares feature extracting pattern.

The coordinates of $P$ are listed in Table 2.3. Note that these coordinates are in the image frame whose origin is at the upper left corner of the image.

Besides the coordinates in the image frame, the coordinates of the same points in the 


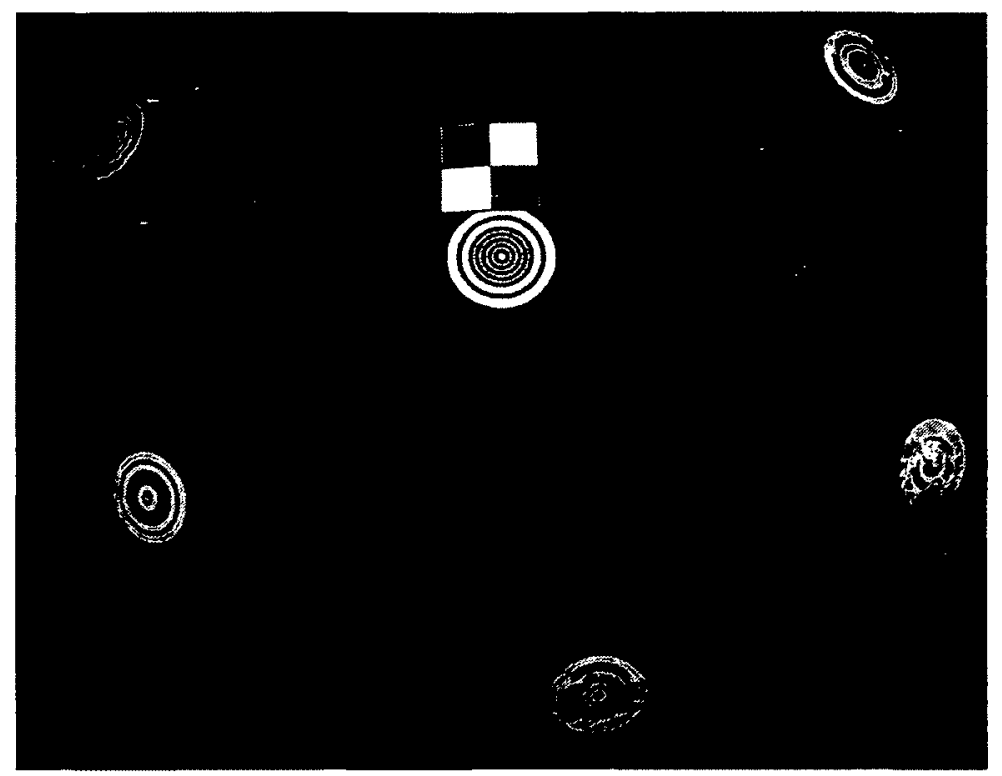

Figure 2.17: Image of the square pattern.

Table 2.3: Coordinates of $P$ of the 4 Squares Pattern in image frame

\begin{tabular}{|l|l|l|}
\hline Number & $\mathrm{X}$ Coordinates & $\mathrm{Y}$ Coordinates \\
\hline$P_{1}$ & 626 & 209 \\
$P_{2}$ & 537 & 579 \\
$P_{3}$ & 1018 & 478 \\
$P_{4}$ & 336 & 572 \\
$P_{5}$ & 217 & 359 \\
$P_{6}$ & 601 & 110 \\
$P_{7}$ & 870 & 65 \\
$P_{8}$ & 437 & 235 \\
$P_{9}$ & 429 & 490 \\
$P_{10}$ & 395 & 641 \\
\hline
\end{tabular}


global frame are also needed. Optotrak is used in order to get these coordinates. The procedure of obtaining the coordinates of these points in the sphere frame are as follow. Note that we name the Optotrak frame as $O_{1}$ and the sphere frame as $O_{2}$ as shown in Figure 2.18. The procedure includes:

1. Measure the coordinates of $P 1$ through $P 10$ in $O_{1}$, named as "Coordiantes Optotrak", as shown in Table 2.4;

2. Measure multiple points on the sphere in $O_{1}$ and compute the coordinates of the geometric centre of the sphere $\left[x_{c}, y_{c}, z_{c}\right]^{T}$ which is used for calculating $H_{t}$;

3. Define the sphere frame $\mathrm{O}_{2}$ in the Optotrak frame $O_{1}$ by calculating the unit vectors $\vec{x}, \vec{y}$, and $\vec{z}$. The first two unit vectors $\vec{x}$ and $\vec{y}$ are calculated by computing the perpendicular vectors against multiple points measured on the $x$ and $z$ planes of $\mathrm{O}_{2}$. The third vector $\vec{z}$ is the cross product of the first two vectors. Note that all the three vectors should go through the sphere center $\left[x_{c}, y_{c}, z_{c}\right]^{T}$ obtained from the previous step;

4. Compute all three unit vectors and calculate the rotation matrix $H_{r}$ between $O_{1}$ and $\mathrm{O}_{2}$

5. Compute the translation matrix $H_{t}$ with $\left[x_{c}, y_{c}, z_{c}\right]^{T}$ obtained from Step 2;

6. Use $H_{r}$ and $H_{t}$ to generate the homogeneous transformation matrix $H$ between $O_{1}$ and $O_{2}$, as shown below;

7. Transform the coordinates in "coordinates optotrak" into corresponding coordinates in $\mathrm{O}_{2}: H \cdot P_{o 1}=P_{o 2}$.

Now the coordinates of $P$ in both of the sphere frame (Table 2.5) and the image frame are obtained, so that the extrinsic parameters can be computed. The OpenCV results are shown below. 


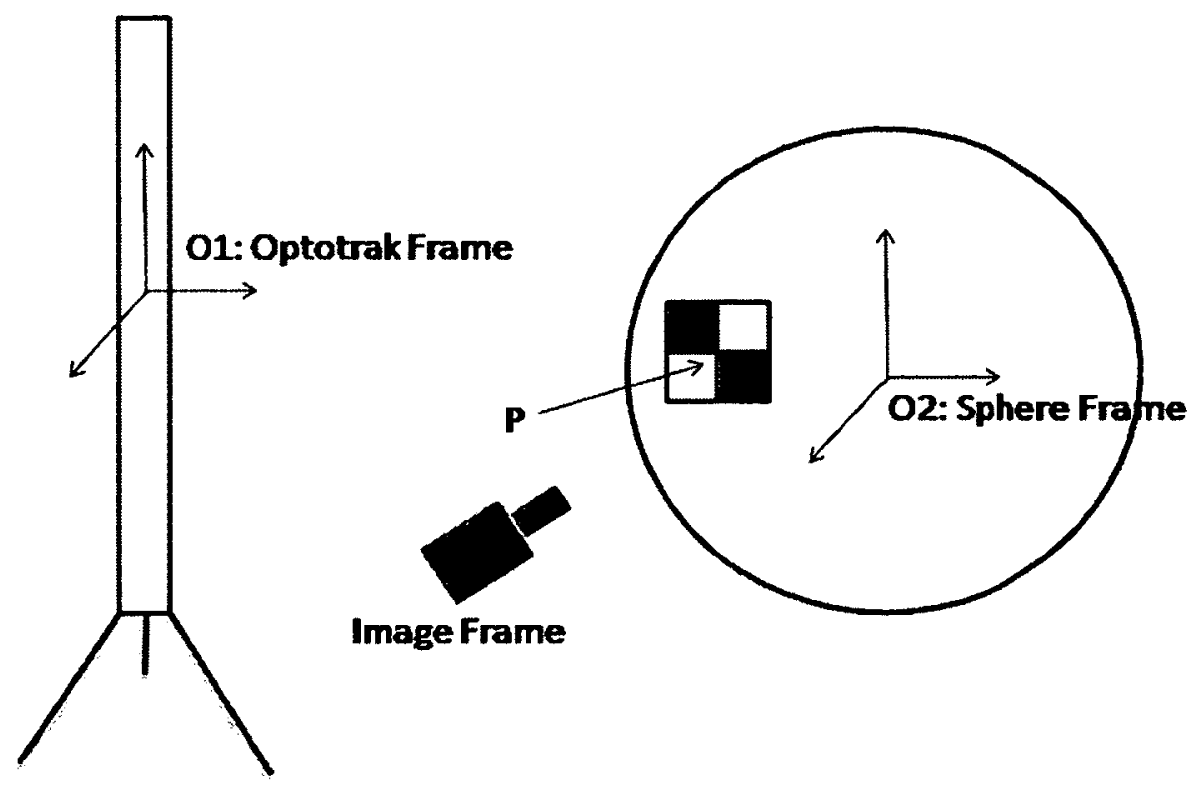

Figure 2.18: Demonstration of the Optotrak frame and the sphere frame.

Table 2.4: Coordinates of $P$ in the Optotrak frame $O 1$.

\begin{tabular}{|l|l|l|l|}
\hline Points & X Coordinates & Y Coordinates & Z Coordinates \\
\hline$P_{1}$ & -29.15 & 482.25 & -3184.86 \\
$P_{2}$ & 37.41 & 571.34 & -3240.66 \\
$P_{3}$ & -108.67 & 545.17 & -3304.93 \\
$P_{4}$ & 99.59 & 563.66 & -3210.86 \\
$P_{5}$ & 117.52 & 503.26 & -3150.59 \\
$P_{6}$ & -31.71 & 450.44 & -3163.38 \\
$P_{7}$ & -116.90 & 433.57 & -3202.64 \\
$P_{8}$ & 30.83 & 483.07 & -3160.32 \\
$P_{9}$ & 59.31 & 548.59 & -3207.55 \\
$P_{10}$ & 88.14 & 581.54 & -3232.99 \\
\hline
\end{tabular}


Table 2.5: Coordinates of $P$ in the sphere frame $O 2$.

\begin{tabular}{|l|l|l|l|}
\hline Points & X Coordinates & Y Coordinates & Z Coordinates \\
\hline$P_{1}$ & -498.47 & 12.02 & -355.22 \\
$P_{2}$ & -418.09 & 43.95 & -445.10 \\
$P_{3}$ & -431.44 & -115.13 & -419.14 \\
$P_{4}$ & -414.45 & 112.80 & -437.31 \\
$P_{5}$ & -458.47 & 157.26 & -376.34 \\
$P_{6}$ & -518.39 & 19.99 & -323.18 \\
$P_{7}$ & -524.61 & -73.64 & -306.45 \\
$P_{8}$ & -491.32 & 76.44 & -355.98 \\
$P_{9}$ & -436.56 & 78.98 & -422.08 \\
$P_{10}$ & -400.60 & 92.19 & -455.38 \\
\hline
\end{tabular}

The unit vectors of $O_{2}$ are: $\vec{x}=\left[\begin{array}{c}0.4786 \\ -0.0107 \\ -0.8779\end{array}\right] ; \vec{y}=\left[\begin{array}{c}0.8780 \\ 0.0022 \\ 0.4786\end{array}\right] ; \vec{z}=\left[\begin{array}{c}-0.0006 \\ -1 \\ 0.0057\end{array}\right]$; The homogeneous transformation matrix $H$ is

$$
H=\left[\begin{array}{cccc}
0.4787 & -0.0053 & -0.8780 & -3278.5 \\
0.8780 & 0.0022 & 0.4786 & 1560.98 \\
-0.00319 & -0.9999 & 0.0104 & 160.1827 \\
0 & 0 & 0 & 1
\end{array}\right]
$$




\section{Calculating the Extrinsic Parameters}

The methodology is still implemented on two platforms here: OpenCV and MATLAB. In OpenCV, the function "FindExtrinsicCameraParams2" is designed for calculating the extrinsic parameters. The inputs are the intrinsic parameters of the camera, the coordinates of the same group of points in both the image frame and the global frame. Finally, the extrinsic matrix calculated by OpenCV is

$$
E=\left[\begin{array}{cccc}
0.028 & -1 & -0.01 & 18.208 \\
0.7 & 0.027 & -0.713 & -4.741 \\
0.713 & 0.013 & 0.701 & 848.284 \\
0 & 0 & 0 & 1
\end{array}\right]
$$

- Errors are inevitable in the procedures of obtaining the extrinsic parameters, they might result from the intrinsic parameters or the process of obtaining the coordinates of points in both of the sphere and the image frames. On the other hand, the approach in OpenCV to model the distortion of the lens is mostly from Doctor Brown [38]. The MATLAB Camera Calibration Toolbox models the distortions based on the model proposed by Janne Heikkil [39]. To ensure the accuracy of the calibration results, another group of extrinsic parameters mis calculated by MATLAB. 
The MATLAB results are,

$$
E=\left[\begin{array}{cccc}
0.0258 & -0.9996 & -0.0136 & 14.0634 \\
0.6997 & 0.0277 & -0.7139 & -5.4776 \\
0.714 & 0.0089 & 0.7001 & 849.1561 \\
0 & 0 & 0 & 1
\end{array}\right]
$$

One way to test the result was to substitute the coordinates of $P$ in the global frame, the intrinsic parameters, and the extrinsic parameters into Equation 2.12, to compute the image frame coordinates. The differences between the calculated values and the original values determine the accuracy of the results. Another two programs were written in both MATLAB and OpenCV to compute the image coordinates and the differences based on the extrinsic and intrinsic parameters obtained from the same software. The calculated values and original values are listed in Table 2.6.

The average absolute differences between the MATLAB results and the original coordinates is 0.6348 pixels, and 0.6610 pixels between the OpenCV results and the original coordinates. According to these results, the extrinsic and intrinsic parameters are reliable.

\subsubsection{Adjustment of the Camera Settings}

In the realtime implementation, the camera takes images of the rotating sphere. The maximum operating angular velocity is $35^{\circ} / \mathrm{sec}$ for the Atlas TDP. When the sphere is rotating, the camera takes images that are prone to blur. Adjustments have to be done to the camera to fit it better to dynamic objects. Therefore, the objective of 
Table 2.6: Calculated Coordinates of $\mathrm{P}$ versus the Original Coordinates

\begin{tabular}{c|c|c|c|c|c|c} 
Points & \multicolumn{2}{|c}{ MATLAB Approach } & \multicolumn{2}{c}{ OPENCV Approach } & \multicolumn{2}{c}{ Original Values } \\
\hline & $\mathrm{X}$ & $\mathrm{Y}$ & $\mathrm{X}$ & $\mathrm{Y}$ & $\mathrm{X}$ & $\mathrm{Y}$ \\
P1 & 626.9 & 207.9 & 626.87 & 208.1 & 626 & 209 \\
P2 & 537 & 578 & 537.2 & 577.9 & 537 & 579 \\
P3 & 1017.8 & 478.6 & 1017.9 & 478.8 & 1018 & 478 \\
P4 & 335.3 & 572.2 & 335.2 & 572.1 & 336 & 572 \\
P5 & 216.1 & 360.3 & 215.9 & 360.2 & 217 & 359 \\
P6 & 601.4 & 110.8 & 601.3 & 110.9 & 601 & 110 \\
P7 & 870.4 & 64.5 & 870.2 & 64.3 & 870 & 65 \\
P8 & 436.6 & 236.3 & 436.6 & 236.4 & 437 & 235 \\
P9 & 428.9 & 489.8 & 429.1 & 489.7 & 429 & 490 \\
P10 & 395.5 & 639.8 & 395.5 & 639.7 & 395 & 641
\end{tabular}

the camera adjustment is to tune the camera hardware settings to reduce the blur on the image. Two solutions were proposed to complete this task, first is to reduce the size of the aperture and the other is to maximize the shutter speed (minimize the shutter time). However, these two solutions lead to lacking of brightness of the images. Usually after the blur is removed from the images, the images are too dark to be processed. A solution to this is setting up a bright lighting system for lumination. Finally, two spot lights were selected and their settings are shown in Table 2.7. Several tests were conducted to find out the best camera settings presented in Table 2.8. With the settings in Table 2.8, the image quality was significantly improved as shown in Figure 2.19. 


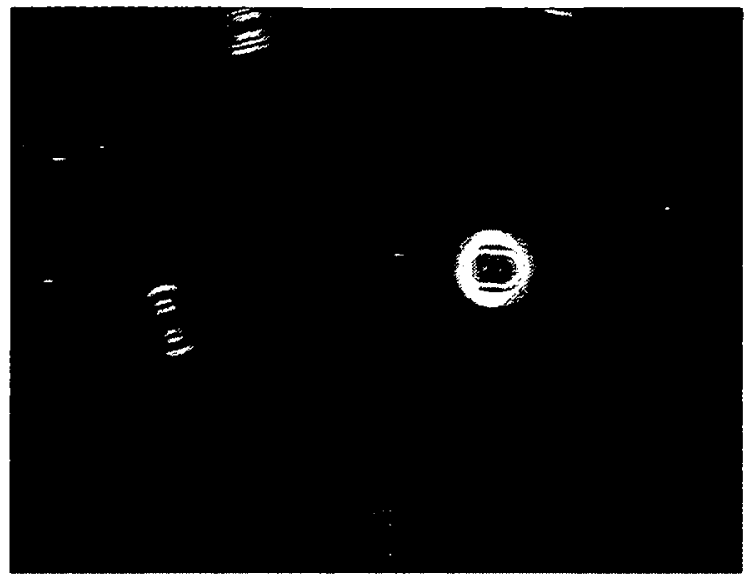

(a) Image with Blur

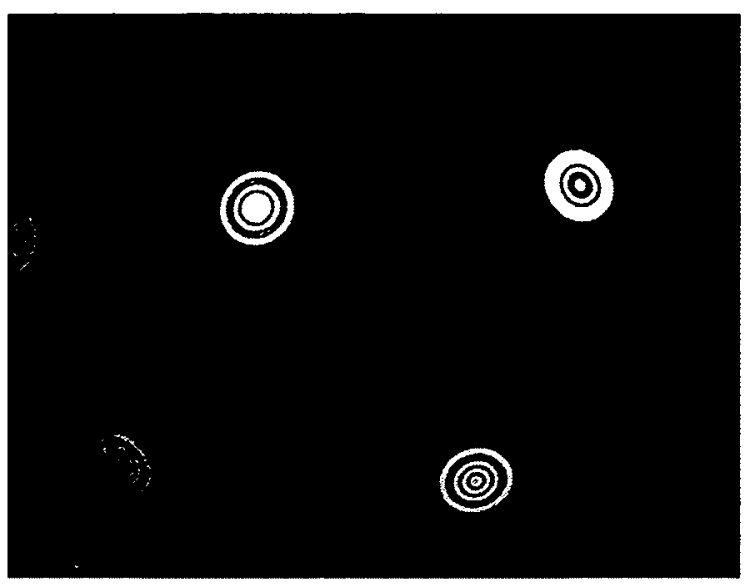

(b) Image without Blur

Figure 2.19: Image quality before and after the camera parameters setting

Table 2.7: Light Settings

\begin{tabular}{|l|l|}
\hline Settings & Value \\
\hline Power & $23 \mathrm{~W}$ \\
Temperature limit & $4100 \mathrm{~K}$ \\
CRI & $>80$ \\
Voltage & $120 \mathrm{~V}$ \\
Frequency & $60 \mathrm{~Hz}$ \\
Current & $348 \mathrm{~mA}$ \\
\hline
\end{tabular}




\subsection{VOS Real-time Implementation}

\subsubsection{Image Processing for Marker Identification}

Upon hardware preparations, the grey scale images with a resolution of $1280 \times 1024$ are taken by an industrial digital camera and sent to an image processing program to identify the marker numbers. This image processing program runs in real time to coordinate with the real-time control. The objective of the image processing algorithm is to identify the marker ID and locations in the image frame using the seven rings. Apparently, comparing the pixel values of a ring with a threshold of 100 (because in a grey scale image, $0=$ black and $255=$ white) is a fast method of distinguishing black and white. To minimize the computation, not all of the pixels on a black or white ring are computed. The pixels located in the middle of a ring would represent the black or white sufficiently. To get the right pixels, the following procedures are used:

1. Image filtering by a threshold which is determined by the lighting conditions of the system, usually an integra that varies from 60 to 80 in the Atlas context.

2. Blobs extraction (where a blob is a set of adjacent points) where in the case of VOS image processing, every blob is a single marker which appears as an ellipse.

3. Blob analysis: extracting the boundary box of the ellipse and computing the locations of the centres of the blobs.

Table 2.8: Camera Settings

\begin{tabular}{|l|l|}
\hline Camera Settings & Value \\
\hline Shutter Speed & 0.0049999 \\
Brightness & -12.43421 \\
Gain & 8.96644 \\
Gamma & 0.89539 \\
\hline
\end{tabular}


4. Determining the pixel locations corresponding to the centre of the blob.

The algorithm is illustrated in Figure 2.20.

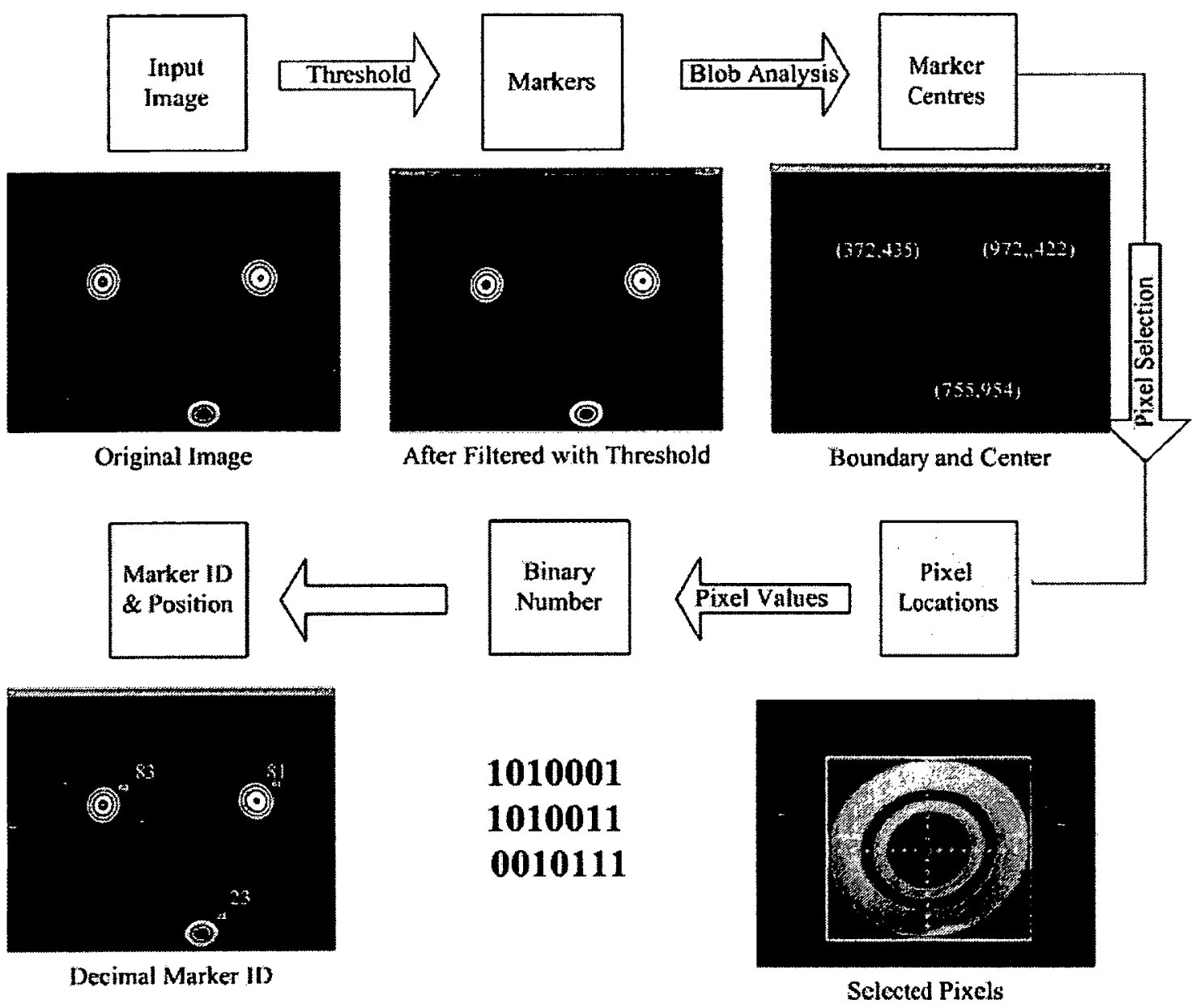

Figure 2.20: Flow Diagram of VOS.

Since the image only contains the $2 \mathrm{D}$ information of the $3 \mathrm{D}$ sphere, distortion is inevitable (markers appear as ellipses). The extent of the distortion increases proportionally with the distance from the centre of the image to the centre of the marker. In addition to the blob analysis, another technique is applied to compensate for the inaccuracy of the centre location determination. Figure 2.21 demonstrates how 
to eliminate distortion effects from the marker locations.

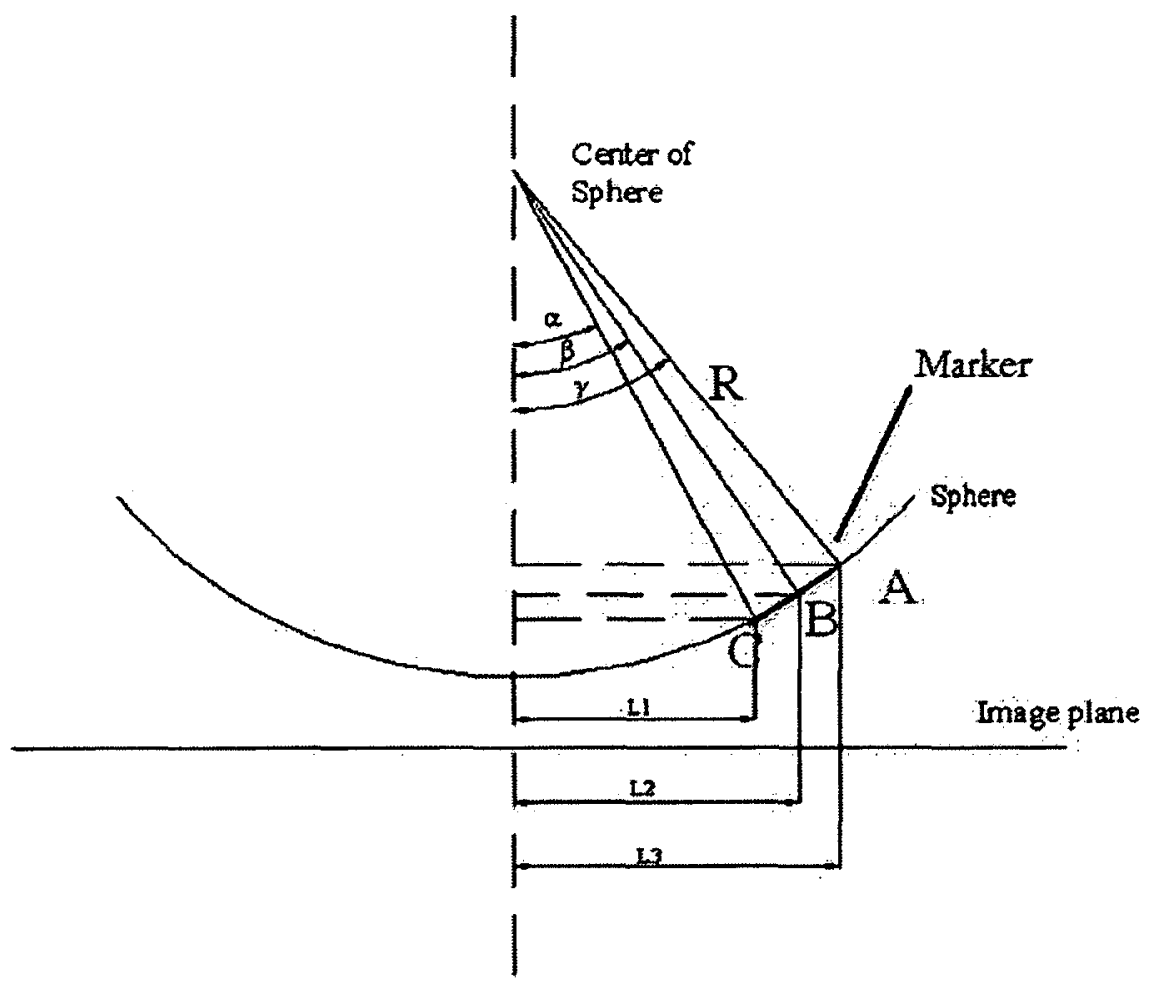

Figure 2.21: Demonstration of centre compensation.

Point $\mathrm{A}$ and $\mathrm{C}$ denote the boundary points of a marker, and point $\mathrm{B}$ is the centre of the marker. The projected distances of the three points from the centre of the sphere are $L_{1}, L_{2}$, and $L_{3}$ respectively. $L_{1}$ and $L_{2}$ are known, while $L_{2}$ is to be calculated to get the position of the real centre of the marker on the image plane. As can be seen from Figure 2.21

$$
\left\{\begin{array}{l}
L_{1}=\mathrm{R} \sin (\alpha) \\
L_{2}=\mathrm{R} \sin (\beta) \\
L_{3}=\mathrm{R} \sin (\gamma) \\
\alpha+\gamma=2 \beta
\end{array}\right.
$$


which leads to

$$
L_{2}=\frac{L_{1}}{\sin (\alpha)} \sin \left(\frac{\alpha+\gamma}{2}\right)
$$

or,

$$
L_{2}=\frac{L_{3}}{\sin (\gamma)} \sin \left(\frac{\alpha+\gamma}{2}\right)
$$

Since point $\mathrm{B}$ is on the same axis as points $\mathrm{A}$ and $\mathrm{C}$, the coordinates of point $\mathrm{B}$ which is the compensated centre can be easily computed as $x$ and $y$ from Equation 2.14 or 2.15. The seven pixel positions corresponding to the centres of the rings forming the marker binary code are calculated as

$$
\begin{cases}x_{i}=x+i D & \text { when } i=1,2,3 \ldots 7 \\ y_{i}=y+i D & \text { when } i=1,2,3 \ldots 7\end{cases}
$$

At least seven pixels which locate on the centre of each ring are selected along the major and minor axes of the marker as shown in Figure 2.22. However, to improve the reliability of the detection, in the real implementation, 28 pixels are picked along the two perpendicular axes through the centre of the marker.

Since we know the value of the 7 pixels, a 7-digit binary number can be calculated by thresholding the pixel values. The threshold is normally set to 100 out of $0-255$. The marker ID which is a decimal number between 1 and 127 is obtained from the binary code embedded in the markers. Two examples can be seen in Figure 2.23.

\subsubsection{Rotation Matrix Calculation}

As in Equation 2.1, to get the rotation matrix from the world frame to the sphere frame, the markers's coordinates $P_{s}$ and $P_{w}$ are needed. Note that $P_{s}$ is a fixed matrix 


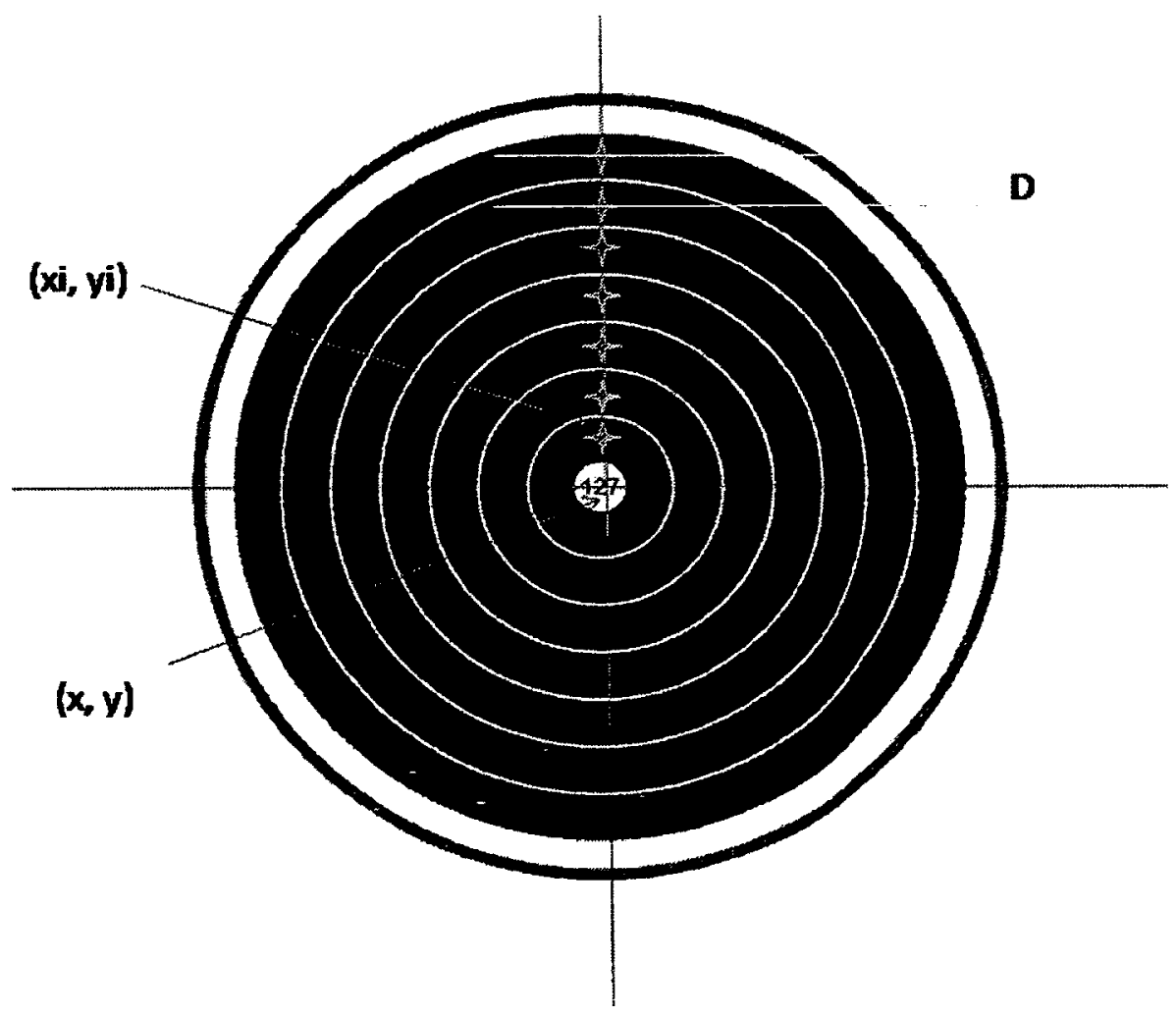

Figure 2.22: Demonstration of pixel selection. 


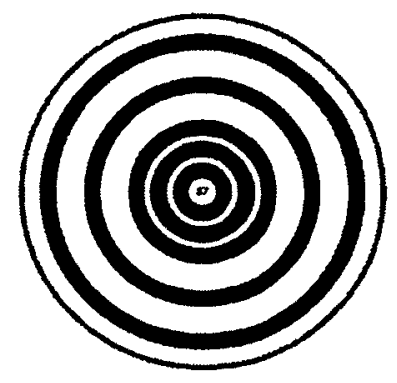

Binary Code: 1010111

Decimal Number: 87

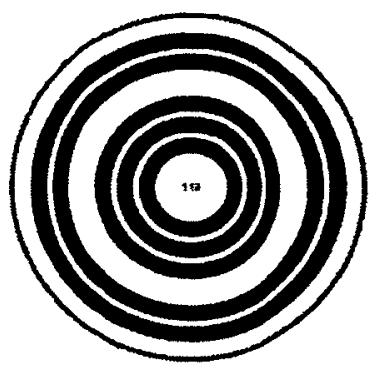

Binary Code: 1101110

Decimal Number: 110

Figure 2.23: Examples of marker identification: Number 87 and Number 110 .

containing the markers's coordinates in the sphere frame, while the $P_{w}=\left[x_{w}, y_{w}, z_{w}, 1\right]^{T}$ is a varying matrix containing the corresponding coordinates in the world frame.

From the image processing program, the centres of the $n$ detected markers and their IDs are obtained with inevitable errors. These errors, as determined from experiments, are from the imperfection in the computation and the distortion of the lens. In order to eliminate the errors, a mathematical method can be conducted to minimize the effect of lens distortion. From the distortion model (presented subsequently Equation 2.19 [39]), a single compensation using the Equations 2.8, 2.9, 2.10, and 2.11 might not remove all of the distortion. To guarantee accuracy, an iterative method is more reliable.

Similar to Equation 2.17, in a pinhole camera model, the location of the marker in the image frame can also be denoted as $(u, v)$. The translation between $(u, v)$ and the 
corresponding coordinates in the camera frame are shown in Equation 2.17 [2].

$$
\left\{\begin{array}{l}
u=u_{0}+f_{x} \hat{x} \\
v=v_{0}+f_{y} \hat{y}
\end{array}\right.
$$

where the $f_{x}$ and $f_{y}$ are the focal length of the lens along and perpendicular to the camera axis, which are exactly the intrinsic parameters that appear in Equation 2.6. The variables $\hat{x}$ and $\hat{y}$ are the distorted image coordinates. This distortion is caused by the lens aberrations as described in Section 2.2.2, and the two most significant distortions are the radial and tangential distortion. The undistorted image coordinates $x$ and $y$ are solved iteratively using [2]

$$
\begin{aligned}
x_{k+1} & =\left(\hat{x}-\left(\delta x_{t}\right)_{k}\right) /\left(\delta x_{r}\right)_{k} \\
y_{k+1} & =\left(\hat{y}-\left(\delta y_{t}\right)_{k}\right) /\left(\delta y_{r}\right)_{k}
\end{aligned}
$$

where the subscript $k$ denotes the iteration index, so that the $\left(x_{k+1}, y_{k+1}\right)$ are the corrected image coordinates of the $k+1$ step of the iteration. The $\left(\delta x_{t}\right)_{k},\left(\delta y_{t}\right)_{k}$, $\left(\delta x_{r}\right)_{k}$, and $\left(\delta y_{r}\right)_{k}$ are the distortion factors which are calculated using the current estimate of the corrected coordinates $\left(x_{k}, y_{k}\right)$ through Equation 2.19

$$
\begin{gathered}
\delta x_{r}=\delta y_{r}=1+k_{1}\left(x^{2}+y^{2}\right)+k_{2}\left(x^{2}+y^{2}\right)^{2} \\
\delta x_{t}=2 p_{1} x y+p_{2}\left(3 x^{2}+y^{2}\right) \\
\delta y_{t}=p_{1}\left(x^{2}+3 y^{2}\right)+2 p_{2} x y
\end{gathered}
$$

The initial distortion factors are calculated with the distorted coordinates. Equation 2.18 is applied iteratively until the change in the estimated undistorted coordinates between iterations is below a specified tolerance [2]. 
Once the image coordinates $(x, y)$ are obtained, they are transformed from $2 \mathrm{D}$ space

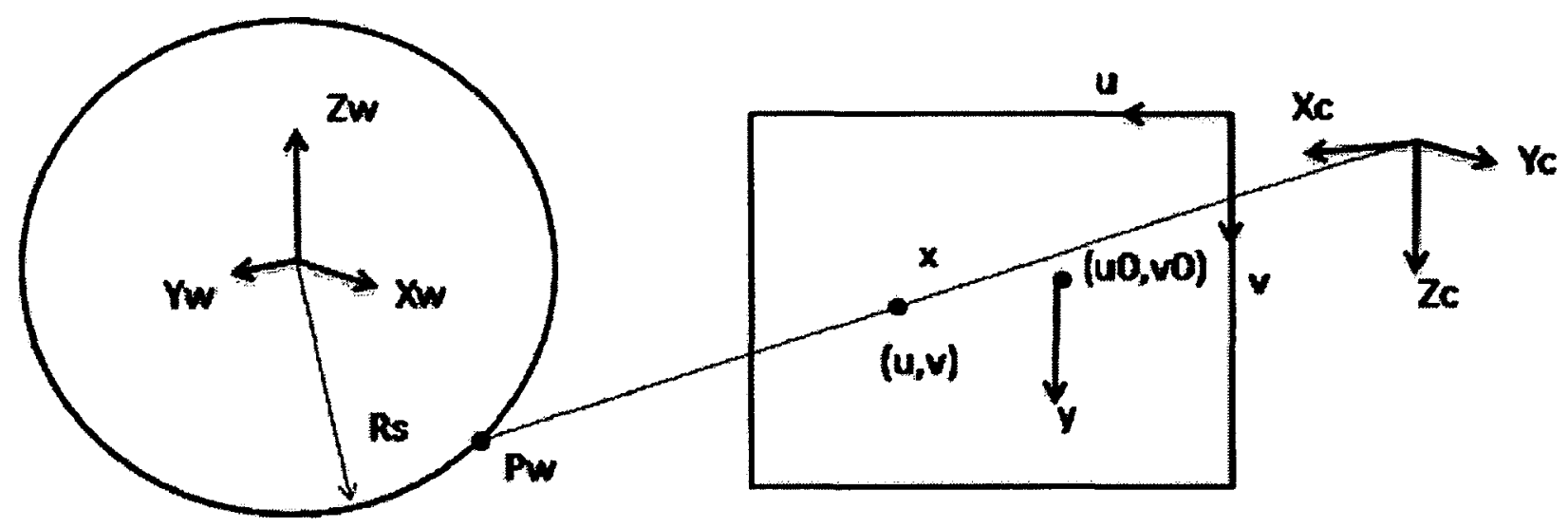

Figure 2.24: Camera Model Demonstration.

to 3D space (the world frame) to get $P_{w}$ as shown in Figure 2.24. Since it is known that all of the markers are on the surface of a sphere of radius $R$, one more constraint is applied stating that:

$$
R_{s}^{2}=x_{w}^{2}+y_{w}^{2}+z_{w}^{2}
$$

Besides the constraint of Equation 2.20, another two equations are needed to solve for $P_{w}$, one of which is the translation from the world frame coordinates to the camera frame coordinates $P_{c}=\left[x_{c}, y_{c}, z_{c}, 1\right]^{T}$ which is

$$
P_{c}={ }^{w} Q_{w} P_{w}
$$


where

$$
{ }^{c} Q_{w}=\left[\begin{array}{cccc} 
& & & \\
& { }^{c} \mathrm{R}_{w} & & { }^{c} \mathrm{~T}_{w} \\
0 & 0 & 0 & 1
\end{array}\right] .
$$

In the above equation, matrix ${ }^{c} \mathrm{R}_{w}$ is the $3 \times 3$ rotation matrix from the world frame to the camera frame. ${ }^{c} \mathrm{~T}_{w}=\left[T_{x}, T_{y}, T_{z}\right]^{T}$ is the translation vector of the world frame origin relative to the camera frame. The other equation shows the transformation between the normalized image coordinates $(x, y)$ and the $P_{c}$ as follows,

$$
\begin{aligned}
& x=\frac{x_{c}}{z_{c}} \\
& y=\frac{y_{c}}{z_{c}}
\end{aligned}
$$

The two solutions of Equation 2.21, 2.23, and 2.20 are solved for a line passing from the focal point through the camera image and intersecting the sphere as illustrated in Figure 2.24. The solution of coordinates for the corresponding point that are closer to the camera frame is selected as the final solution as given by,

$$
P_{w}={ }^{c} \mathrm{R}_{w}^{T}\left\{\begin{array}{c}
x \zeta+x T_{z}-T_{x} \\
y \zeta+y T_{z}-T_{y} \\
\zeta
\end{array}\right\}
$$

where $\zeta=\left(x^{2}+y^{2}+1\right)^{-1} x T_{x}+y T_{y}-T_{z}\left(x^{2}+y^{2}\right)-\left[r^{2}\left(x^{2}+y^{2}+1\right)-\left(T_{x}-x T_{z}\right)^{2}\right.$ $\left.-\left(T_{y}-y T_{z}\right)^{2}-\left(y T_{x}-x T_{y}\right)^{2}\right]^{1 / 2}$.

Now both $P_{w}$ and $P_{s}$ are available and the rotation matrix can be calculated using Equation 2.1. 


\subsubsection{VOS Testing on the Real-time Platform}

The algorithm described above has been tested in three computational environments such as: MATLAB, OpenCV, and LABVIEW. However, since the VOS program has to be implemented on the LABVIEW Real-time Operating System (RTOS) and only the LABVIEW program is compatible with the RTOS, only the LABVIEW version was tested in real time. The program runs on a desktop computer with the LABVIEW Real-time Operating System. The software runs at $18 \mathrm{~Hz}$ with a Intel Core i5 processor. The user interface of the LABVIEW program is shown in Figure 2.25.

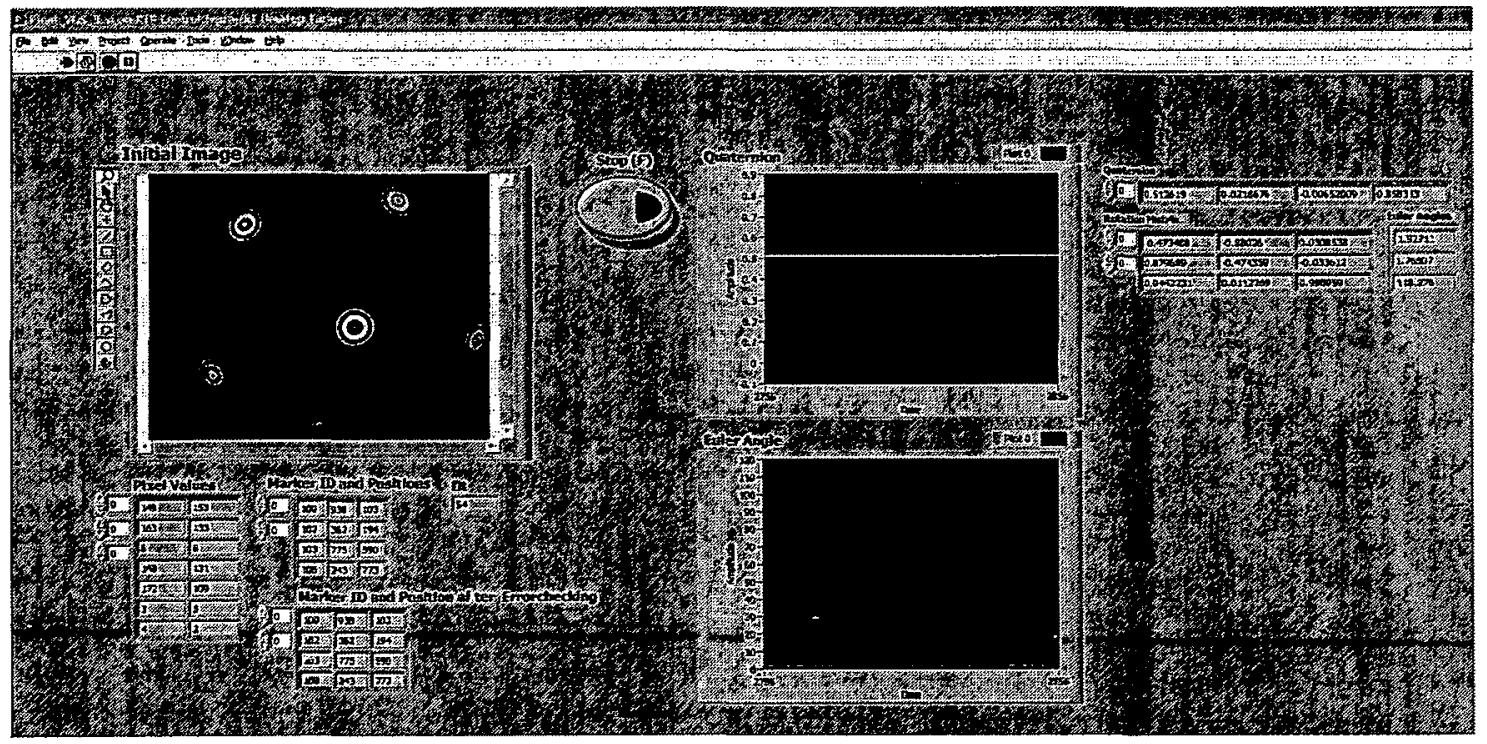

Figure 2.25: LABVIEW VOS program GUI.

To verify the performance of the VOS, several experiments were carried out. In these experiments, the sphere was manually rotated for $120^{\circ}$ using the motor (which is separated from each other in $120^{\circ}$ ) as the reference for 10 times, and the measured orientation obtained from the IOS and VOS are recorded. The rotation angle contains an error of less than $1^{\circ}$. The result of VOS and IOS are displayed in Figure 2.26. It is evident from the plot that the orientation measured by the VOS is far more accurate than the IOS. It also verifies that the VOS measurement is totally independent of 
time. The average rotation value of the VOS measurement is $120.8^{\circ}$ while the IOS measurement is $109.7^{\circ}$.

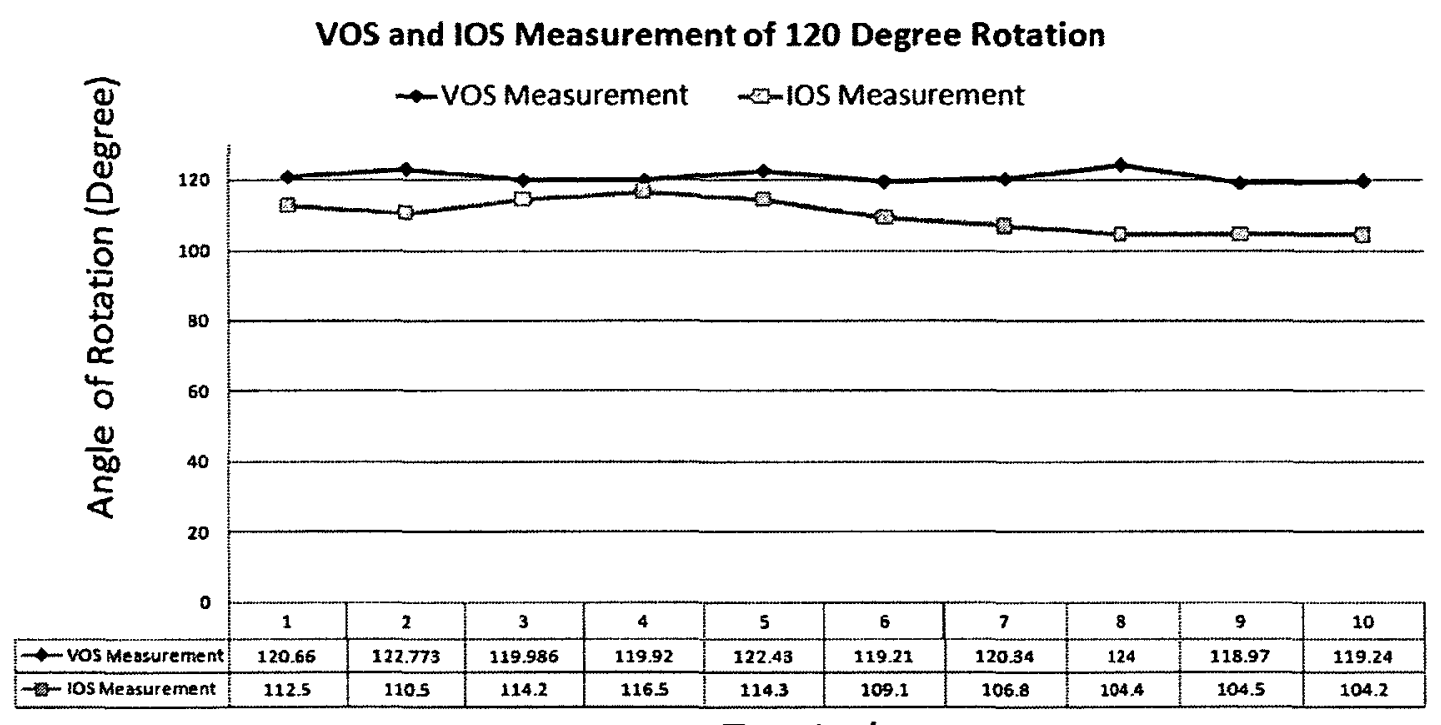

Test Index

Figure 2.26: VOS and IOS measurement of a rotation of $120^{\circ}$.

\subsubsection{Conclusion}

This chapter described the process of establishing a vision-based orientation sensor. The work includes the system preparations which are the marker design and their distribution on the sphere, the camera calibration, and the real time implementation. Several tests were conducted to verify the performance of the VOS. Compared with the IOS, the VOS has following advantages:

1. More precise measurement;

2. Longer usage time; and 


\section{No error accumulation}

However, there still exist a few drawbacks of the VOS. These are the relatively slower frequency and the need for a robust and steady lighting system. When the lighting condition changes, the pixel values could be different such that the markers will not be detected correctly. Overall, the VOS is found to satisfy its role of compensating for the errors in the IOS measurement. 


\section{Chapter 3}

\section{Vision-based Orientation Control}

As stated in the introduction, the control problem of Atlas is to ensure the actual angular velocity of the sphere tracks its desired value. In the case of the Atlas TDP, the controller is only focused on controlling the orientation of the sphere, as the translation and rotation are decoupled. However, the orientation of the sphere is difficult to control due to the complex and highly nonlinear omniwheel-sphere contact dynamics. The performance of controllers implemented in the past on Atlas has been limited due to the sensing limitations which have been previously discussed. The new developed sensing system (VOS) has enabled the implementation of a nonlinear controller [2] on the Atlas TDP. This particular controller has demonstrated limited ability for tracking when implemented on the non-real-time Atlas Lite system. The control scheme of the Atlas TDP follows the same design as the controller proposed for Atlas Lite. However, the feedback of the controller is provided by the newly-developed VOS to compensate for slip effects associated with the mecanum wheels. The overall control structure is shown in Figure 3.1, where $q_{d}$ and $q_{m}$ are the unit quaternions representing the desired and measured orientations of the sphere respectively, $e$ is the difference between $q_{d}$ and $q_{m}, \Omega_{r}$ is the reference angular velocity of the sphere, and $\omega_{r}$ is the reference angular velocity of the three motors. The error $e$ is sent to the quaternion-based controller to generate the referenced angular velocity of the sphere which will be converted to the 
angular velocity input to the motor control system. The motor control program uses the angular velocity to produce torque to actuate the Atlas platform. These variables will be further clarified through implementation and the experiments performed with a LABVIEW real-time computing platform. This quaternion-based nonlinear controller will be shown effective in the control of the Atlas TDP platform in this chapter.

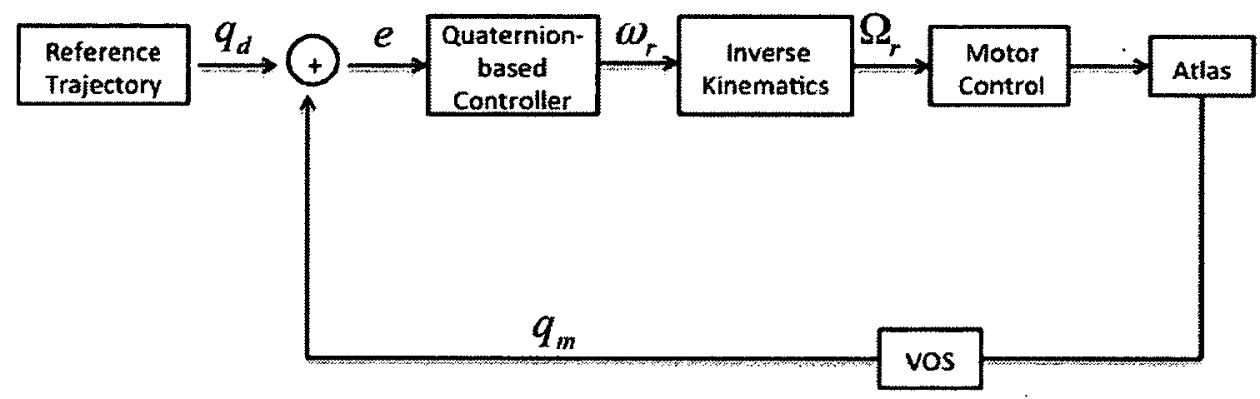

Figure 3.1: Block diagram of the orientation controller for the Atlas TDP.

\subsection{Nonlinear Quaternion-based Controller}

As mentioned in Chapter 1, unit quaternions are used for representing the orientation of the sphere. Variables $q_{d}$ and $q_{m}$ are the desired quaternion and the measured quaternion respectively. They are defined as,

$$
\begin{gathered}
q_{m}=q_{m, 0}+q_{m, 1} \vec{i}+q_{m, 2} \vec{j}+q_{m, 3} \vec{k}=q_{m, 0}+\vec{q}_{m} \\
q_{d}=q_{d, 0}+q_{d, 1} \vec{i}+q_{d, 2} \vec{j}+q_{d, 3} \vec{k}=q_{d, 0}+\vec{q}_{d}
\end{gathered}
$$

where $q_{m, 0}$ and $q_{d, 0}$ correspond to the scalar components of each unit quaternion, $\vec{q}_{m}$ and $\vec{q}_{d}$ correspond to the vector components of each unit quaternion [2]. The time 
derivative of Equation 3.1 are,

$$
\begin{gathered}
\dot{q}_{m}=\frac{1}{2} \omega_{m} q_{m} \\
\dot{q_{d}}=\frac{1}{2} \omega_{d} q_{d}
\end{gathered}
$$

where $\omega_{m}$ represents the angular velocity of the sphere and $\omega_{d}$ represents the desired angular velocity of the sphere. Note that both of these two vectors are also represented in the quaternion forms as [2],

$$
\begin{gathered}
\omega_{m}=0+\omega_{m, x} \vec{i}+\omega_{m, y} \vec{j}+\omega_{m, z} \vec{k}=0+\vec{\omega}_{m} \\
\omega_{d}=0+\omega_{d, x} \vec{i}+\omega_{d, y} \vec{j}+\omega_{d, z} \vec{k}=0+\vec{\omega}_{d}
\end{gathered}
$$

where $\omega_{m, x}, \omega_{m, y}$, and $\omega_{m, z}$ are the components of the sphere's angular velocity with respect to the global coordinate frame, while $\omega_{d, x}, \omega_{d, y}$, and $\omega_{d, z}$ are the components of the desired sphere angular velocity with respect to the same global frame.

The tracking error of the orientation is represented by the difference between the desired quaternion and the measured quaternion defined by

$$
e \equiv q_{d}-q_{m}
$$

Note that the $e$ in Equation (3.4) does not have a physical meaning, but is providing a measurement of the tracking error of the orientation. The error dynamics is formulated from Equation 3.4 as:

$$
\dot{e}=\dot{q}_{d}-\dot{q}_{m}=\frac{1}{2} \omega_{d} q_{d}-\frac{1}{2} \omega_{m} q_{m}
$$

In the above equation, $\omega_{m}$ is selected to achieve the desired error dynamics such that

$$
\omega_{m}=\omega_{n o m}=\left(\omega_{d} q_{d}+2 k e\right) q_{m}^{-1}
$$


where $\omega_{n o m}$ is a quaternion representing the nominal reference sphere angular velocity and $k$ is a user-selectable control gain. The error dynamics will be reduced to $\dot{e}=-k e$; so that the asymptotic convergence of the orientation tracking error is easy to prove [2]. It is very important to note that the quaternion $\omega_{\text {nom }}$ is not physically realizable if its scalar part is nonzero since the $\omega_{r}$ (reference angular velocity of the sphere) will be converted into the reference angular velocity $\Omega_{r}$ of the three motors through inverse kinematics. A solution to this is to redefine the $\omega_{r}$ as:

$$
\omega_{r}=\omega_{n o m}-\omega_{n o m, 0}=0+\vec{\omega}_{n o m}
$$

where $\omega_{n o m, 0}$ and $\vec{\omega}_{n o m}$ are the scalar and the vector parts of the nominal angular velocity of the sphere.

Equation (3.7) ensures the scalar part of the reference angular velocity remains zero. The vector part of the reference angular velocity will be decomposed to the desired angular velocity of the three mecanum wheels $\left(\Omega_{r}\right)$. If the angular velocity controller of the actuators is able to track the reference angular velocity of each mecanum wheel, the real angular velocity of the sphere $\omega_{m}$ is able to track the desired $\omega_{r}$ [2].

If we assume that $\omega_{m}=\omega_{r}$, then substitute Equation 3.7 into Equation 3.5 , the error dynamics will reduce to $\dot{e}=-k e+\frac{\omega_{n o m, 0}}{2} q_{m}$ or:

$$
\begin{aligned}
& \dot{e}_{0}=-k e_{0}+\frac{\omega_{\text {nom }, 0}}{2} q_{m, 0} \\
& \dot{e}_{1}=-k e_{1}+\frac{\omega_{\text {nom }, 0}}{2} q_{m, 1} \\
& \dot{e}_{2}=-k e_{2}+\frac{\omega_{n o m, 0}}{2} q_{m, 2} \\
& \dot{e}_{3}=-k e_{3}+\frac{\omega_{n o m, 0}}{2} q_{m, 3}
\end{aligned}
$$

While the controller of Equation 3.8 does not guarantee asymptotic convergence of the 
error to zero, it can be shown in [2] that the error will be uniform ultimate bounded, i.e., the orientation tracking error $e$ is guaranteed to be bounded and the size of the bound can be controlled through the selection of the control gain $k$. From [2], the norm of the orientation tracking error $e$ may be expressed as,

$$
\|e\| \leq \frac{\epsilon}{2 k}
$$

where $\epsilon$ denotes the norm of the maximum desired sphere angular velocity $\vec{\omega}_{d}$. Therefore, the size of the bound is inversely proportional to $k$. To minimize the bound of $e, k$ should be as large as possible; however, $k$ is limited by the physical conditions of the Atlas platform as the slippage happens with the large torque that can be produced by the motors. An optimal $k$ is selected after tests which are introduced in the following sections.

\subsection{Controller Implementation on the LABVIEW RT Platform}

The controller proposed in [2] is selected as the design for the Atlas TDP, which is programmed in LABVIEW and tested with step inputs, sinusoidal inputs, and the flight simulator input from a commercial software package to determine the performance of the controller. These tests can be generally divided into single-DOF tests, multi-DOF tests and the flight simulator data tests.

To coordinate with the entire real-time system, the controller runs as a single process in LABVIEW named Control_q_vos.vi. The block diagram of the structure of the control system is shown in Figure 3.2. The controller process, the VOS process, and the digital camera run at the same frequency of $18 \mathrm{~Hz}$ on the RTOS. While the 


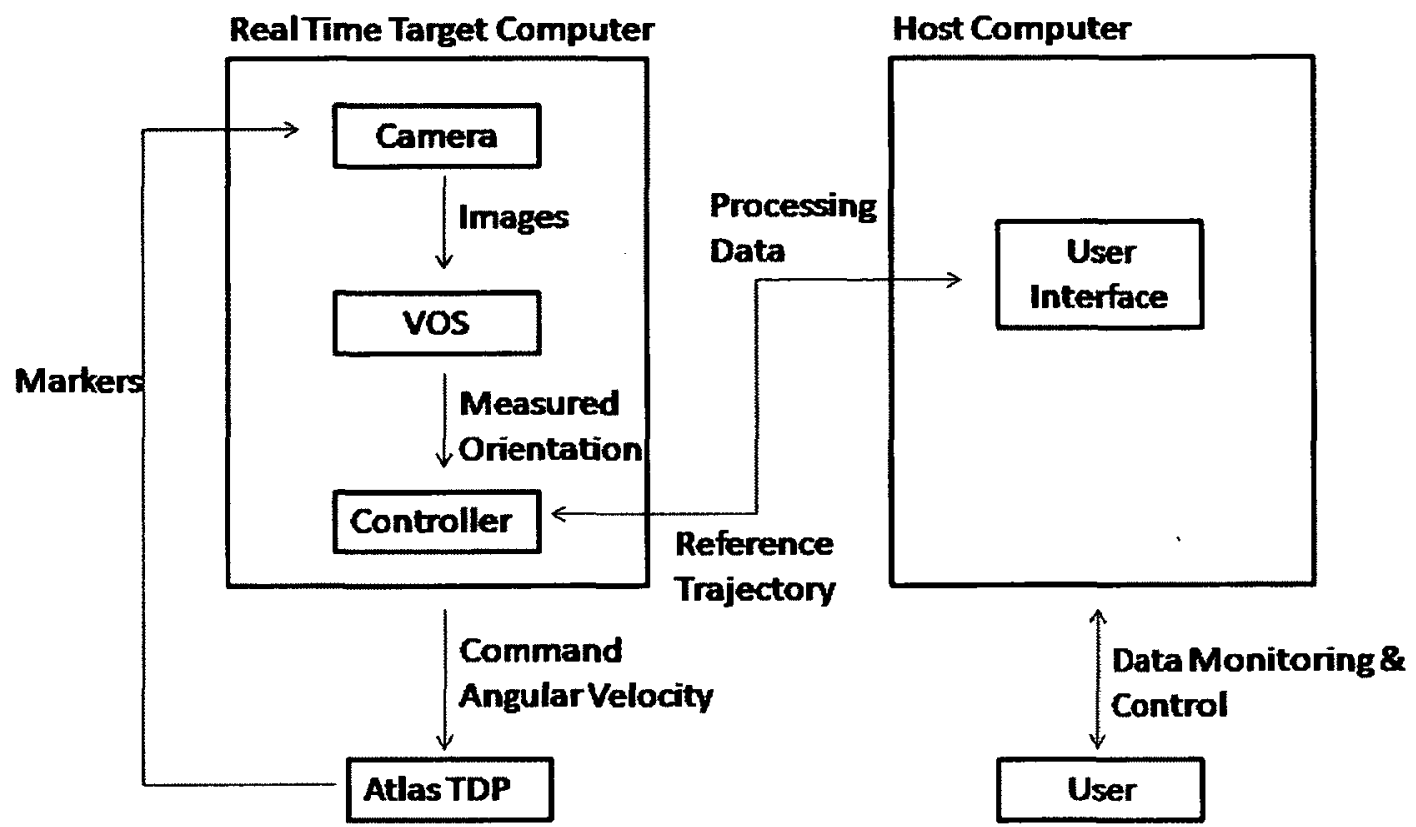

Figure 3.2: The GUI of the LABVIEW Control Program. 
graphical user interface (GUI) is displayed on the host computer for communication with the user. The camera takes grey scale images of markers on the sphere surface and sends them to the VOS. The measured orientation from the VOS is sent to the controller to produce the angular velocities of the sphere which are then converted to the angular velocities of the motors. The Atlas TDP is then actuated so that new images will be taken by the camera to repeat the same procedures for the next time increment.

The GUI of the controller program is as shown in Figure 3.3. All of the measured and reference trajectories (yaw, pitch, and roll) are displayed in the three graphs in Figure 3.3. Once the program is running, all of the processed data are also displayed on the user interface for monitoring and can be recorded for later analysis.

The orientation and the angular velocity of the input signal have to be in the unit quaternion format when sent into the controller. To have a physically meaningful input to this control program, the step and the sinusoidal inputs are specified using Euler angles. This angular velocity will then be converted into the unit quaternion format through:

$$
q=\left[\begin{array}{c}
\cos (\phi / 2) \cos (\theta / 2) \cos (\varphi / 2)+\sin (\phi / 2) \sin (\theta / 2) \sin (\varphi / 2) \\
\sin (\phi / 2) \cos (\theta / 2) \cos (\varphi / 2)-\sin (\phi / 2) \sin (\theta / 2) \sin (\varphi / 2) \\
\cos (\phi / 2) \sin (\theta / 2) \cos (\varphi / 2)+\sin (\phi / 2) \cos (\theta / 2) \sin (\varphi / 2) \\
\cos (\phi / 2) \cos (\theta / 2) \sin (\varphi / 2)-\sin (\phi / 2) \sin (\theta / 2) \cos (\varphi / 2)
\end{array}\right]
$$

where $\phi, \theta$, and $\varphi$ denote the euler angle roll, pitch, and yaw Euler angles respectively. The step input is built as a vector of three components representing the angles around the yaw, pitch, and roll axes. In addition to step inputs, sinusoidal inputs and typical flight trajectories are also used to test the controller performance. The initial 


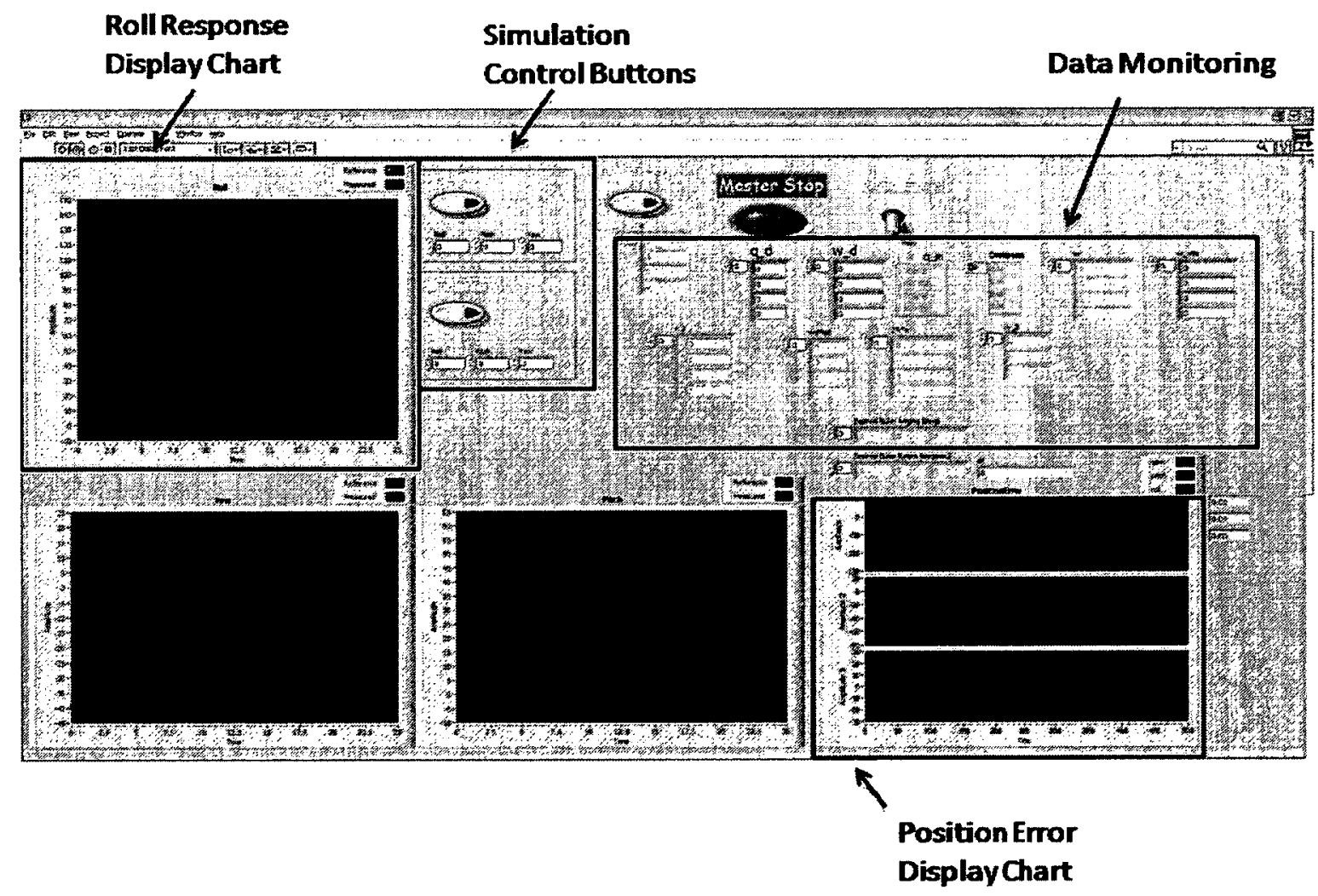

Figure 3.3: The GUI of the LABVIEW Control Program. 
simulation data is collected from Microsoft Flight Simulator 2004 over a certain period of time which is usually 60 seconds. The input data are converted into an array in LABVIEW. While the simulation is processing, its values are read from the array in every control loop.

It is important to note that in the control program, the feedback orientation signals are obtained and sent by the VOS which was introduced in Chapter 2. Therefore, the VOS program runs simultaneously with the control program and shares the orientation data with the controller. In LABVIEW, there are two built-in solutions to share data between different VIs: the shared variable node and insertion of a VI as a function. From several tests of the two different approaches, it was found that the shared variable data would delay the data transportation compared to embedding the VOS VI into the control program. Finally the flow of information through the system control loop over the time line is shown in Figure 3.4, where each step contains several processes running at the same time. The time period of the control loop which is also named as sample time of the controller is restricted by the processing time of the VOS which is approximately $54 \mathrm{~ms}$ (corresponding to $18 \mathrm{~Hz}$ ). This $54 \mathrm{~ms}$ is mainly the limit imposed by the frame rate of the camera, which is 18.5 frames per second.

During a step of the control loop, the first step is to generate the desired trajectory and capture the image, which is demonstrated as STEP 1 in Figure 3.4. The desired orientation data are then sent to the controller for the computation of motor commands. Meanwhile, the image of the markers on the sphere is captured by the camera and then sent to the VOS program which is embedded in the control program. The measured orientation data will then be sent to the controller to compute the command angular velocity. In the last step, motors take the command from the controller to rotate the sphere into the desired position. 


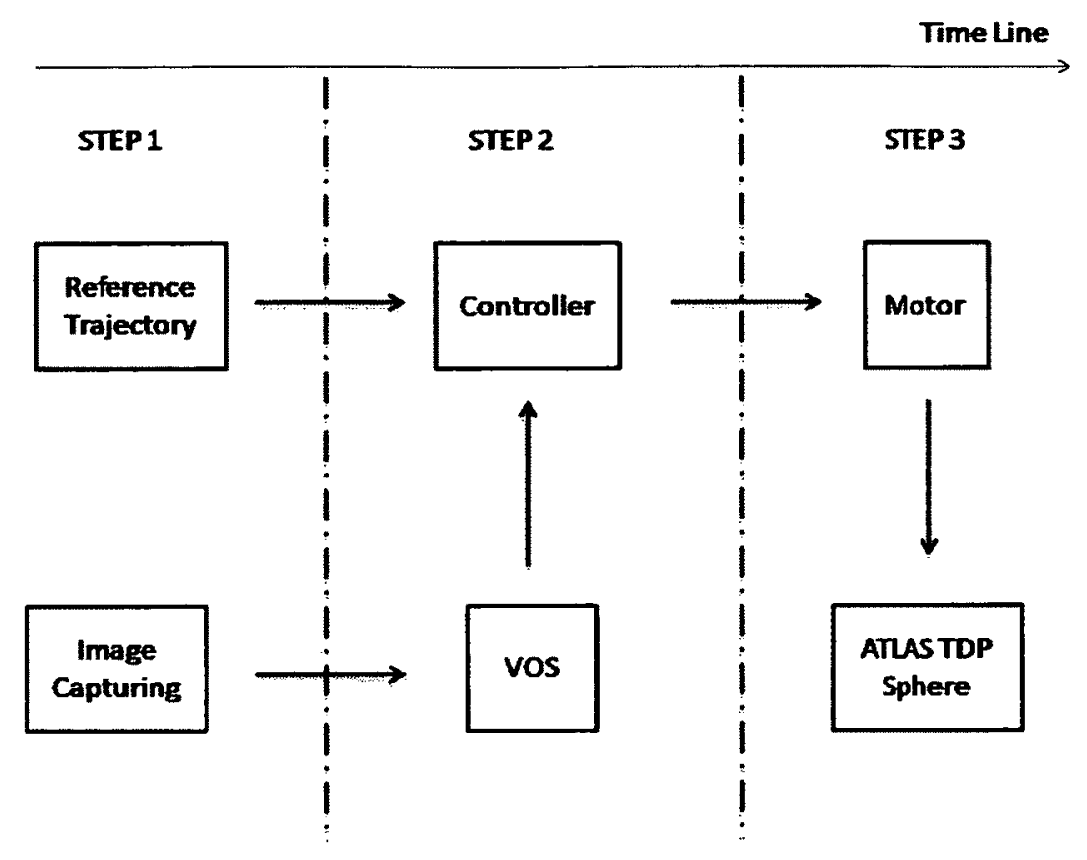

Figure 3.4: Flow Chart of the TDP system over Timeline.

\subsection{Gain Scheduling Controller}

The entire architecture of the controller is built in a "timed loop" in LABVIEW which is designed to precisely control the timing of a loop structure. After the controller was first successfully implemented in LABVIEW, tests were conducted to test the program and the control architecture, as well as to tune the control gain $k$ (see Equation 3.11). From the tests, however, it is discovered that $k$ is hard to determine for meeting the requirements of the controller. If $k$ is less than 4 , settling time is approximately 5 seconds; if the $k$ is larger than 8 the system ends up in severe oscillation, because the system is a slow responding system that cannot follow up the fast change of orientation while reaching steady state. There is a trade-off in selecting the appropriate gain for the controller. Large $k$ (larger than 8 ) is desirable for smaller steady state error and faster response, while it can adversely affect the stability. This problem was presented in [2]. To achieve a good balance between the steady-state errors and the stability 
issue, we use a variable gain controller with a gain schedule dependent on the amount of the error is used. Also, large gains create large motor torques when the errors are large which result in saturation. This is not acceptable since the slippage appears more easily when the sphere experiences large acceleration from the torque. Overall, a fixed gain would result in the following issues (Note that all the images show the response about roll axis, dashed line denotes the measured trajectory, and the solid line denotes the reference trajectory):

1. A fixed small gain leads to slow response when the error is large (see Figure 3.5) and resulting bad performance.

2. A fixed small gain result unsaturation when the error is small (see Figure 3.6) and bad performance.

3. A fixed small gain produces no slippage when the error is large (see Figure 3.5) and resulting good performance.

4. A fixed large gain creates slippage (the overshoot) between the wheels and the sphere (see Figure 3.7) and resulting bad performance.

5. A fixed large gain leads to instability when the error is small (see the steady state in Figure 3.7) and resulting bad performance.

6. A fixed large gain leads to fast response and saturation when the error is large (see Figure 3.7) and 3.8 and resulting good performance.

Thus, a solution to the gain challenge is to schedule the gain. When the error between the desired orientation and the measured orientation is over a threshold, the gain remains a small constant. When the error reduces, the gain increases inversely proportional to the error. The new gain between the gain $k$ and the error $e$ is denoted 


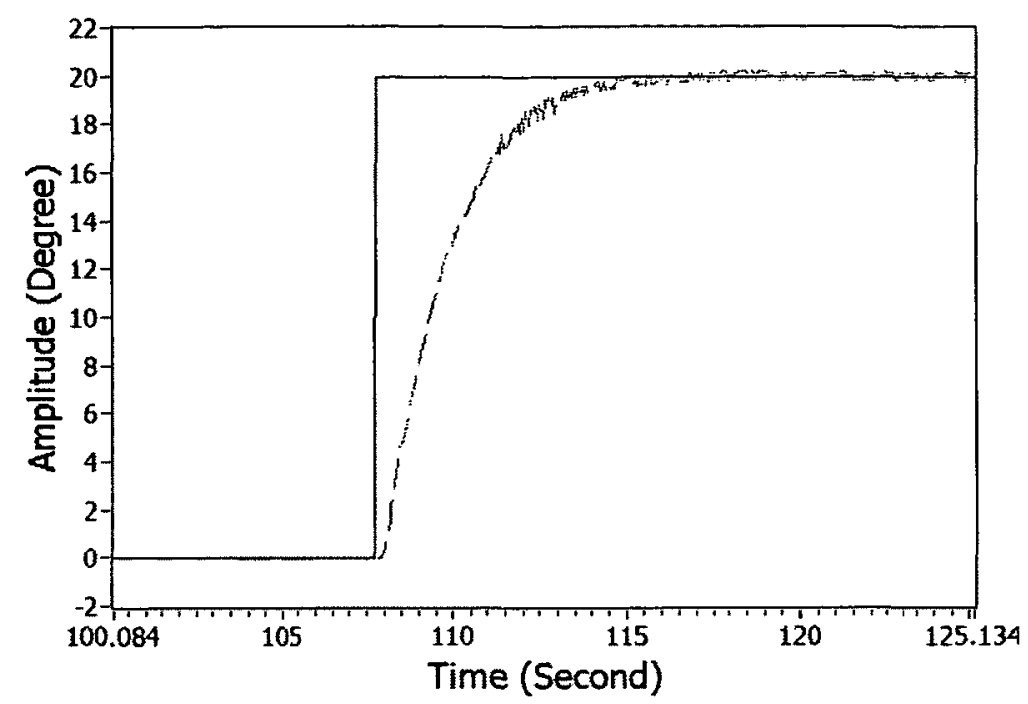

Figure 3.5: Performance of $k=1$ when $e$ is large.

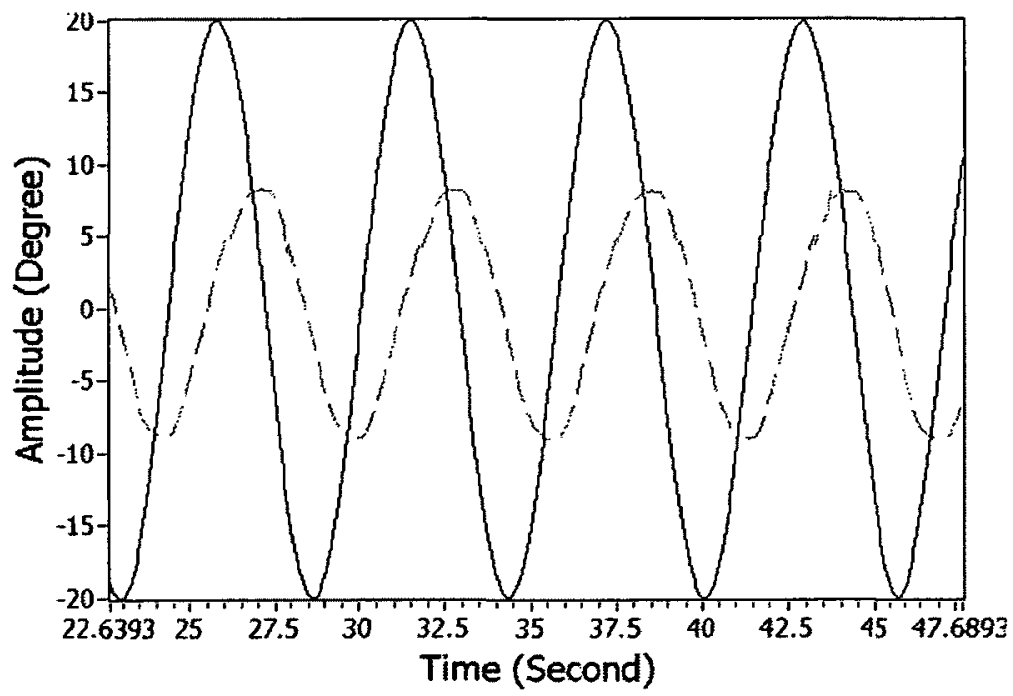

Figure 3.6: Performance of $k=1$ when $e$ is small. 


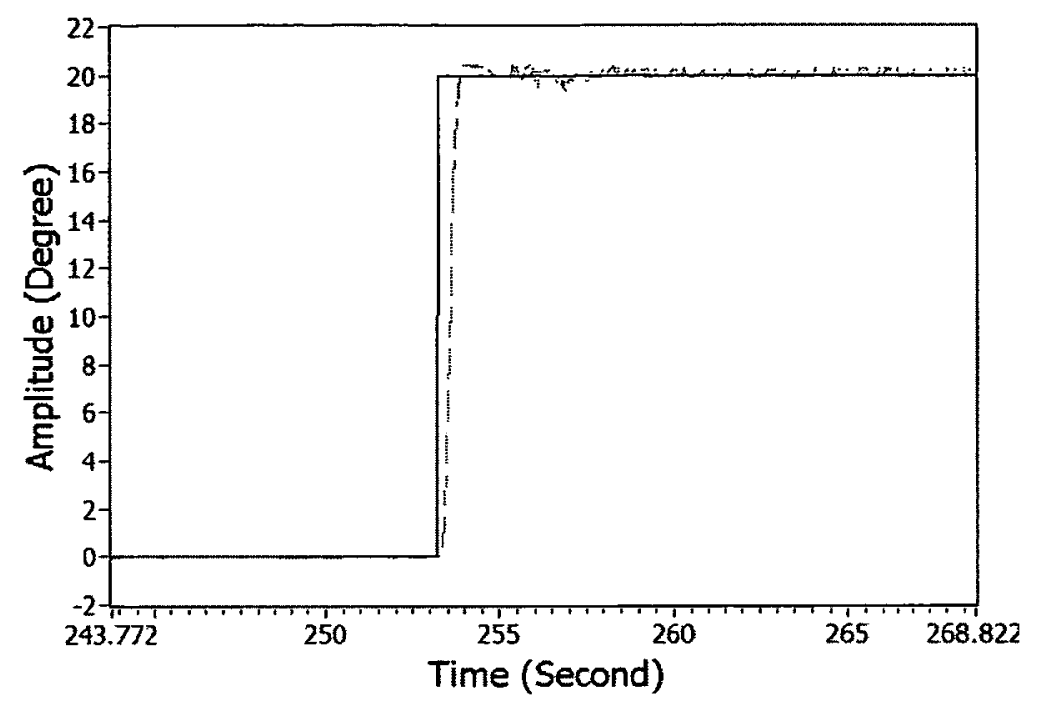

Figure 3.7: Performance of $k=8$ when $e$ is large.

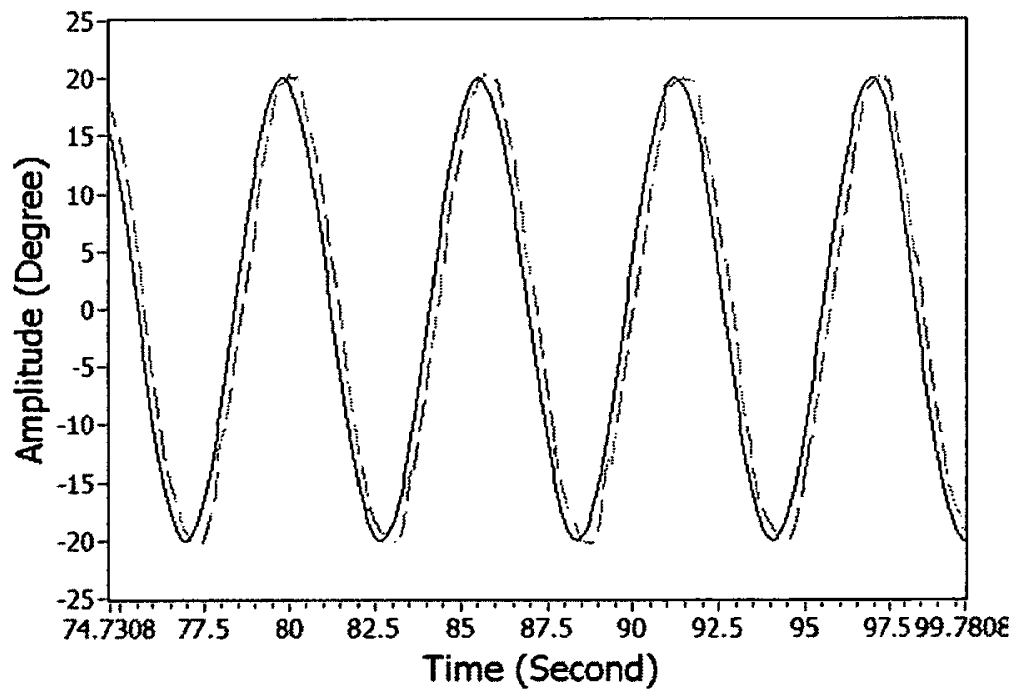

Figure 3.8: Performance of $k=8$ when $e$ is large. 
as $g$ here. The new definition of the gain $k$ is,

$$
\begin{aligned}
& k=20 g+c \quad \text { if }|e| \geq 20^{\circ} \\
& k=e \cdot g+c \quad \text { if }|e|<20^{\circ}
\end{aligned}
$$

where $c$ is a constant and $g$ is the scaling value for $e$. To find out the upper and lower boundaries, several tests were conducted, in which $k$ was varied from 1 to 9 . Therefore, $k$ is finally defined as in Figure 3.9, where $g=-0.4$ and $c=9$. This gain $k$ has proved to be suitable for the tested inputs such as the step and sinusoidal inputs, as it gets rid of the oscillation and also decreases the response time of the controller.

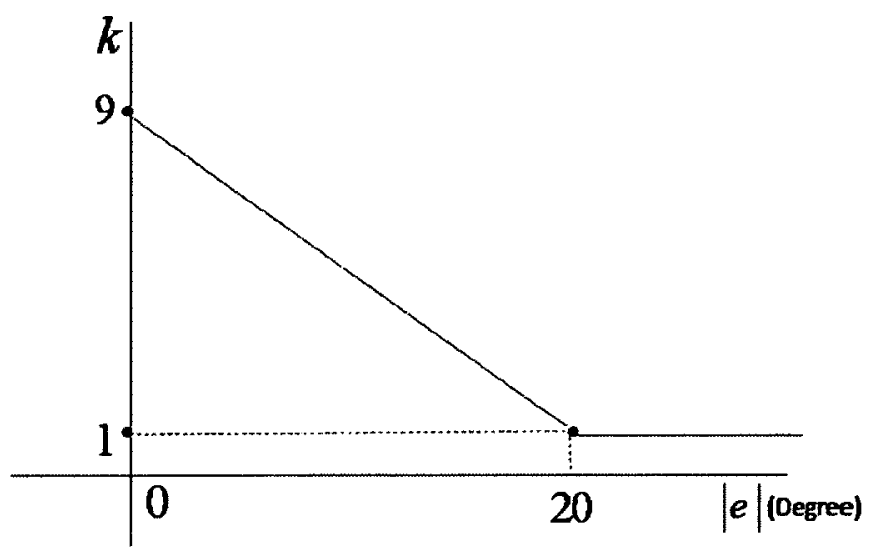

Figure 3.9: Definition of the gain $k$. 


\subsection{Controller Test Results}

Three groups of tests were conducted with the real-time implementation of the Atlas TDP. The first group of tests involved commanding the simulator to rotate around a single axis (yaw/pitch/roll) denoted as the single-DOF tests. The second group of tests involved commanding the simulator to rotate around multiple axes (two or three axes). The last group involved rotating the sphere as prescribed by the data collected from the Flight Simulator software. Figure 3.10 shows the response of a $10^{\circ}$ step input and Figure 3.11 shows the response of a sinusoidal input with an amplitude of $10^{\circ}$ and a frequency of $0.1 \mathrm{~Hz}$. Note that the dashed line denotes the measured trajectory and the solid line denotes the reference trajectory in all the figures.

Figure 3.10 shows the sphere can track the desired input of $10^{\circ}$ about any of the yaw, pitch and roll axes. The average steady state error is less than $0.01^{\circ}$ with an overshoot of less than $5 \%$ (overshoot is only observed on pitch and roll). The settling time is approximately $0.5 \mathrm{~s}$. The step response of the Atlas TDP varies between yaw, pitch, and roll motions and this variation can be attributed to various reasons such as the imperfection of the sphere's geometry, the friction between the sphere and the mecanum wheels, as well as the motors. The results from Figure 3.11 show the sphere tracking a desired sinusoidal trajectory over 25 seconds. The largest absolute error between the desired orientation and the measured orientation is $3.3^{\circ}$ and the root mean square errors are less than $2.5^{\circ}$ which mostly resulted from the delay of the mechanical system. If the time shift is removed, the RMS error drop to around $0.1^{\circ}$ as shown in Table 3.1 and Figure 3.12 . 


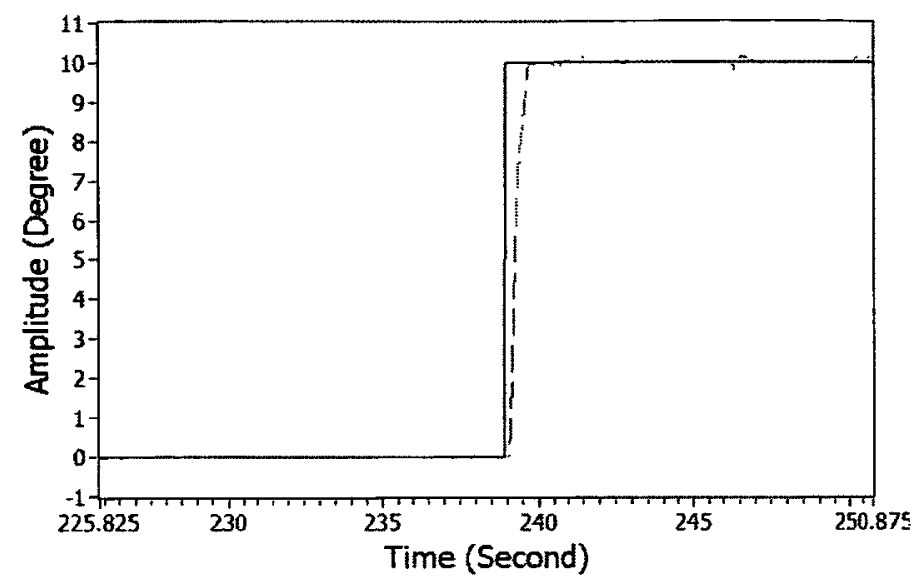

(a) Yaw

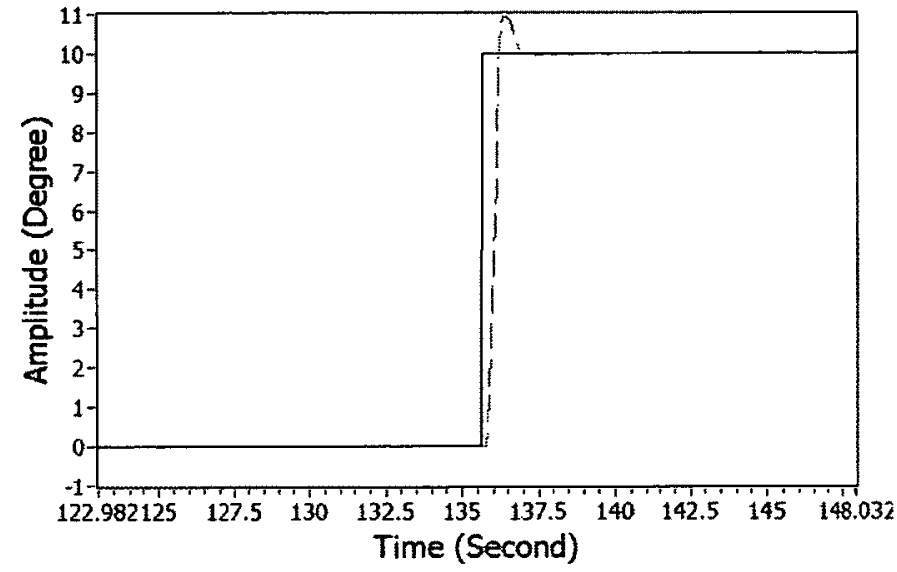

(b) Pitch

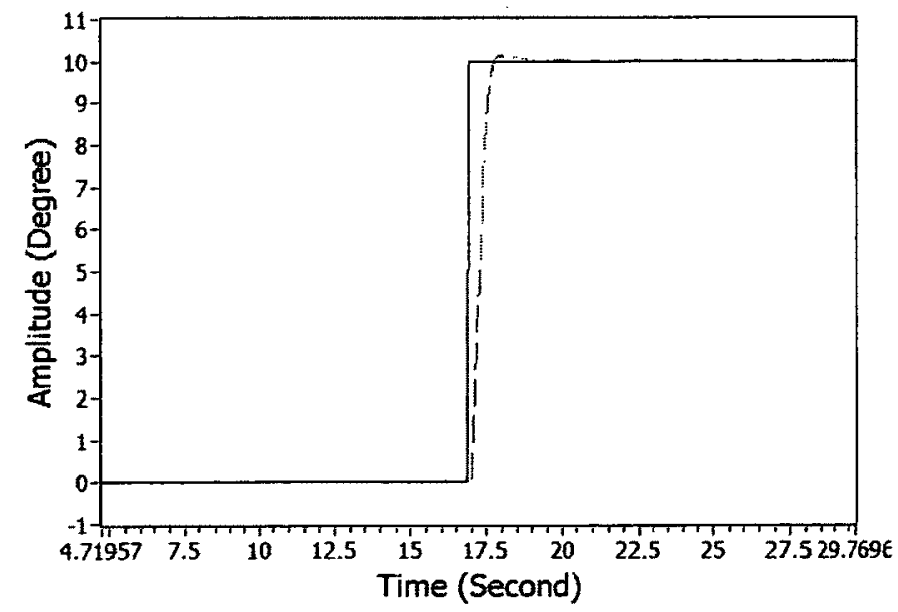

(c) Roll

Figure 3.10: Single-DOF step response of a $10^{\circ}$ input. 


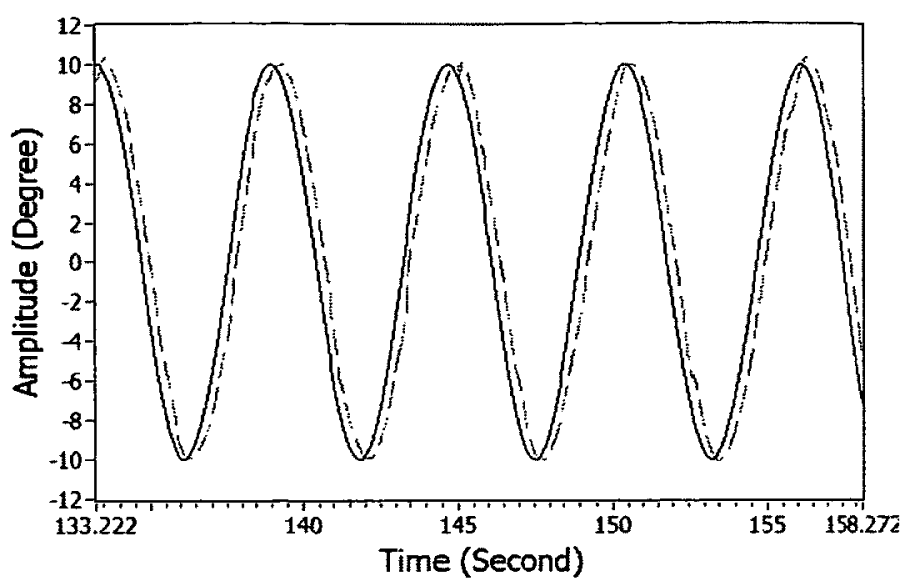

(a) Yaw

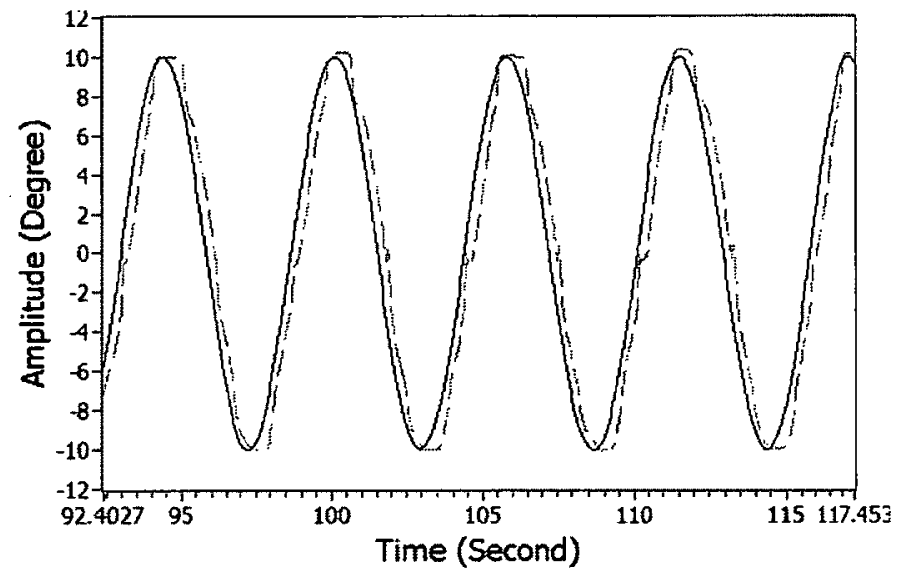

(b) Pitch

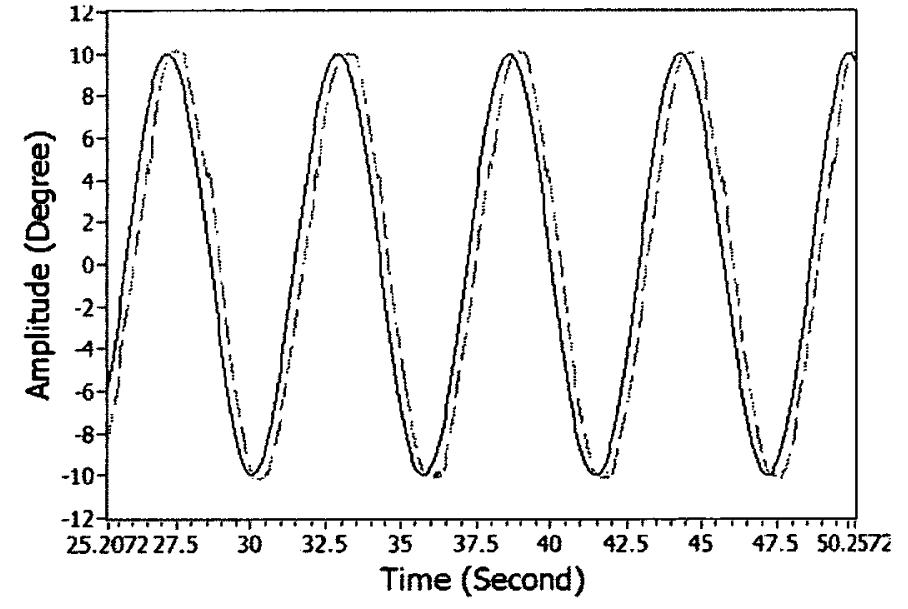

(c) Roll

Figure 3.11: Single-DOF sinusoidal response of a $10^{\circ}$ input. 


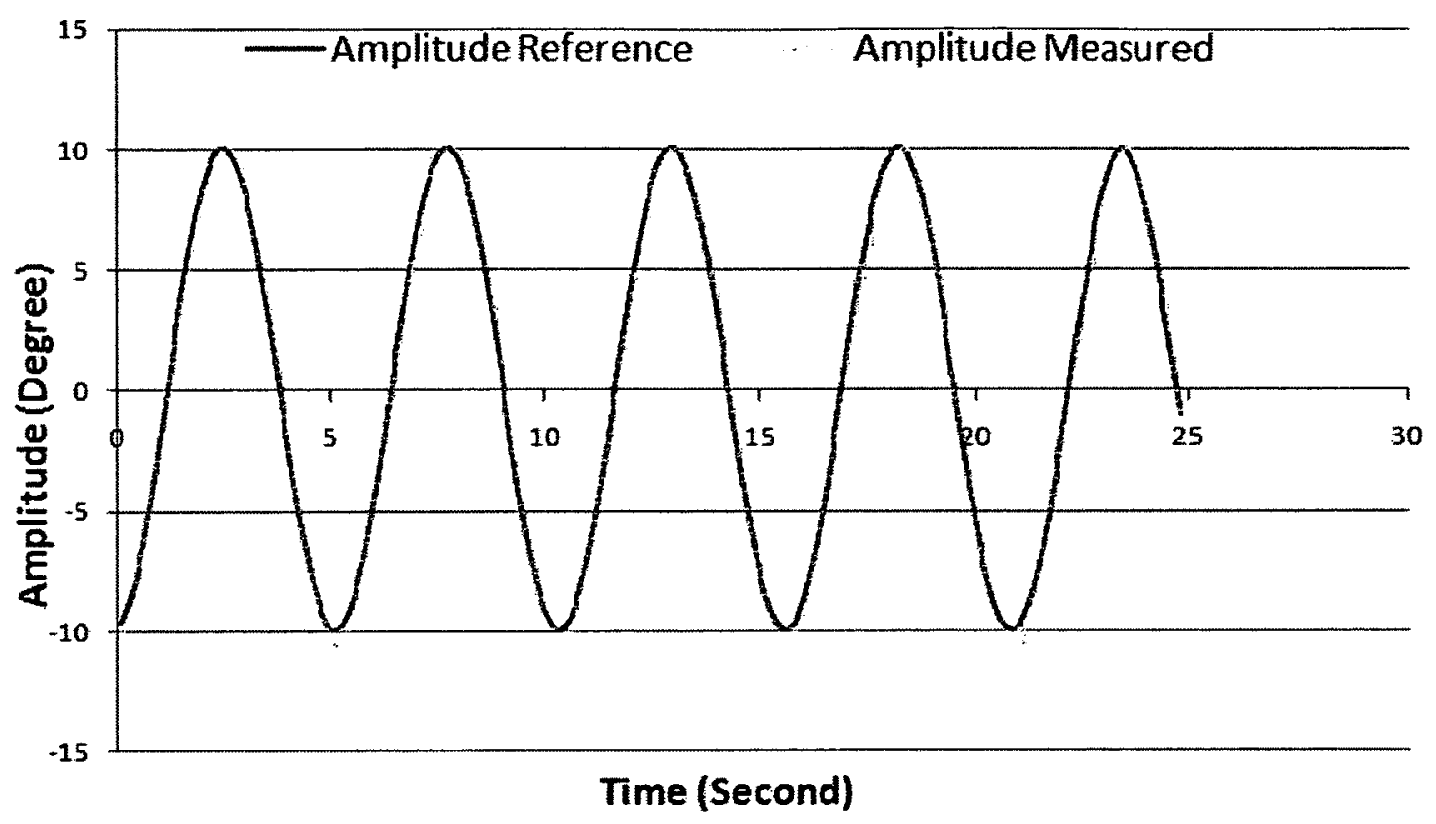

Figure 3.12: Sinusoidal response on roll of a $10^{\circ}$ input without time shift

Table 3.1: RMS error of single-DOF sinusoidal response without time shift

\begin{tabular}{cc}
\hline Axis & RMS Error \\
\hline Yaw & $0.317^{\circ}$ \\
Pitch & $0.667^{\circ}$ \\
Roll & $0.362^{\circ}$ \\
\hline
\end{tabular}

It is also observed that the response about yaw is the most smooth one because of the dynamical difference from pitch and roll due to the specific way the mecanum wheels are arranged. When the sphere is rotating about yaw, all three motors are rotating in the same direction at the same speed with no transverse motion across any mecanum wheels [2]. This favourable condition reduces the dynamic effect of the mecanum wheels such as the potential of slippage. The two other motions around roll and pitch are combined with a little noise and oscillation. Both of the testing results of the step response and the sinusoidal response can be seen in Tables 3.2 and 3.3 . 
Table 3.2: Steady state error of single-DOF step response

\begin{tabular}{cc}
\hline Axis & Steady State Error \\
\hline Yaw & $0.01^{\circ}$ \\
Pitch & $0.01^{\circ}$ \\
Roll & $0.01^{\circ}$ \\
\hline
\end{tabular}

Table 3.3: RMS error and maximum error of single-DOF sinusoidal response

\begin{tabular}{ccc}
\hline Axis & RMS Error & Maximum Error \\
\hline Yaw & $1.91^{\circ}$ & $3.30^{\circ}$ \\
Pitch & $1.76^{\circ}$ & $3.32^{\circ}$ \\
Roll & $2.33^{\circ}$ & $3.30^{\circ}$ \\
\hline
\end{tabular}

Besides the single-DOF tests, the multi-DOF tests of the same step and sinusoidal response are conducted and the results are shown in Figures 3.13 and 3.14. It can be seen that the controller can track the desired trajectory even in a $3 \mathrm{D}$ motion. The steady state error of the multi-DOF step response and the RMS error and the maximum error of the multi-DOF sinusoidal response are shown in Tables 3.4 and 3.5 respectively.

Table 3.4: Steady State Error of Multi-DOF Step Response

\begin{tabular}{cc}
\hline Axis & Steady State Error \\
\hline Yaw & $0.00^{\circ}$ \\
Pitch & $0.01^{\circ}$ \\
Roll & $0.00^{\circ}$ \\
\hline
\end{tabular}




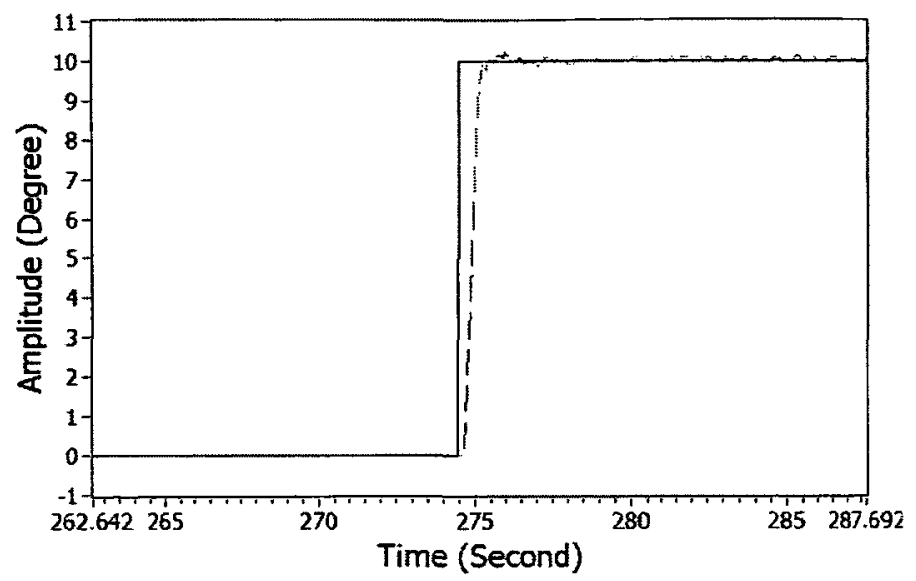

(a) Yaw

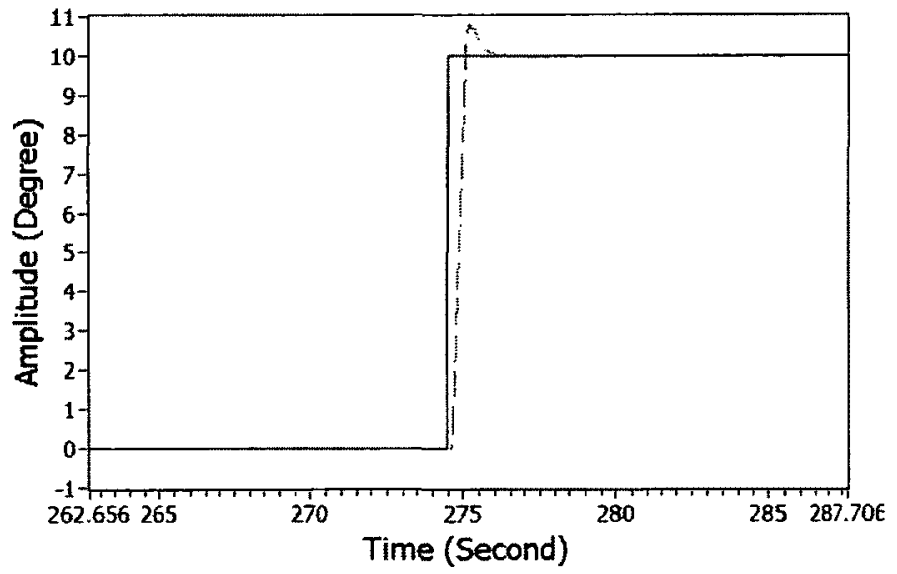

(b) Pitch

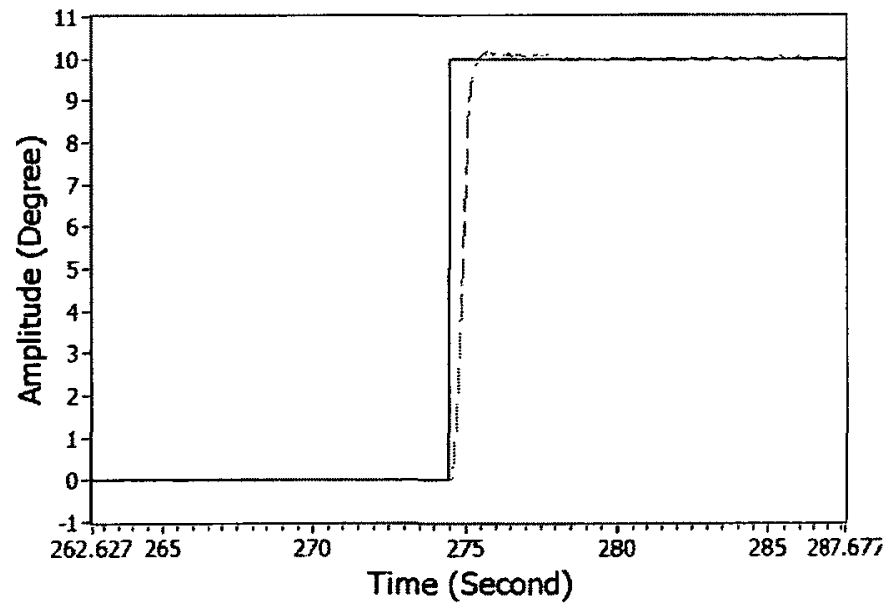

(c) Roll

Figure 3.13: Multi-DOF step repones of a $10^{\circ}$ Input 


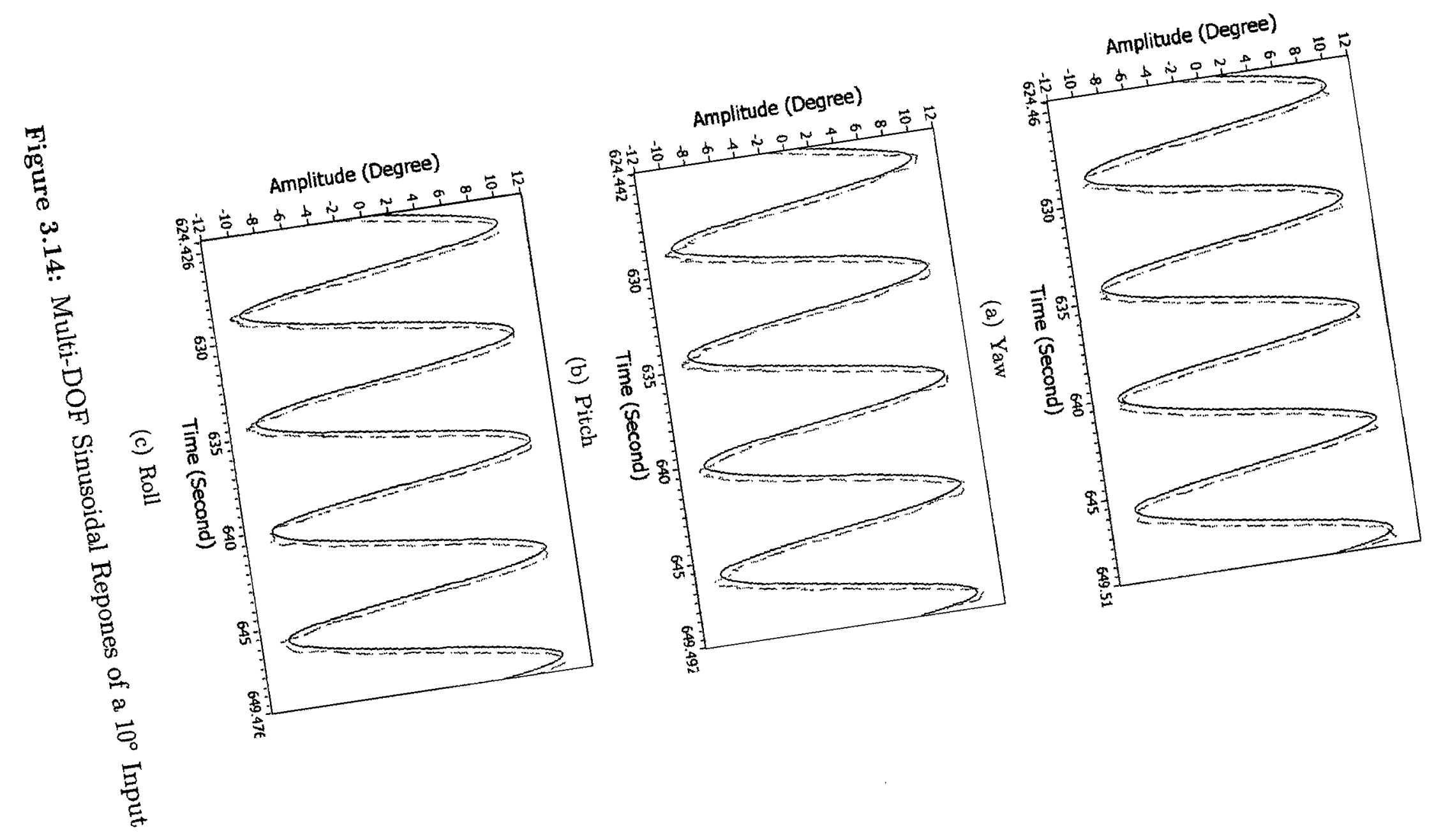


Table 3.5: RMS Error and Maximum Error of Multi-DOF Sinusoidal Response

\begin{tabular}{ccc}
\hline Axis & RMS Error & Maximum Error \\
\hline Yaw & $2.32^{\circ}$ & $4.20^{\circ}$ \\
Pitch & $2.07^{\circ}$ & $3.50^{\circ}$ \\
Roll & $2.01^{\circ}$ & $3.07^{\circ}$ \\
\hline
\end{tabular}

Compared to the single-DOF tests, the steady state error of the step response remains insignificant, which indicates that the system would always converge to the desired state no matter whether the motion is single-DOF or multi-DOF. On the other hand, the RMS errors of the multi-DOF sinusoidal responses are around $0.4^{\circ}$ larger than the RMS errors of the single-DOF sinusoidal responses. The reason for this might be that as the system executing more complex motions than the single-DOF, more frictions and slippage are introduced. It is also noticed that when the system gets to the position of roll $=10^{\circ}$, yaw $=10^{\circ}$, and pitch $=10^{\circ}$ (transient points), some oscillations are observed. This might come from the fact that when the system is getting to the transient point where its angular velocity is close to zero and acceleration is the highest, the stiction forces are significantly affecting the system. The VOS measurements would also contribute to the oscillations, since the readings are not always stable and include tiny step changes while different combinations of markers are used to calculating the rotation matrix (see Figure 3.15). This issue is fixed by filtering VOS and IOS, which will be introduced in Chapter 4 .

Finally, the flight simulator data collected when the simulated "Cessna Skyhawk 172SP" aircraft was flown by a trained pilot was also tested on the controller. This trajectory represents a real flight including take-off and cruising. 25 second period of the flight simulator test results is shown in Figure 3.16. An Atlas TDP sphere could track the real pattern of the simulation with a maximum error of $12.8^{\circ}$ and a RMS 


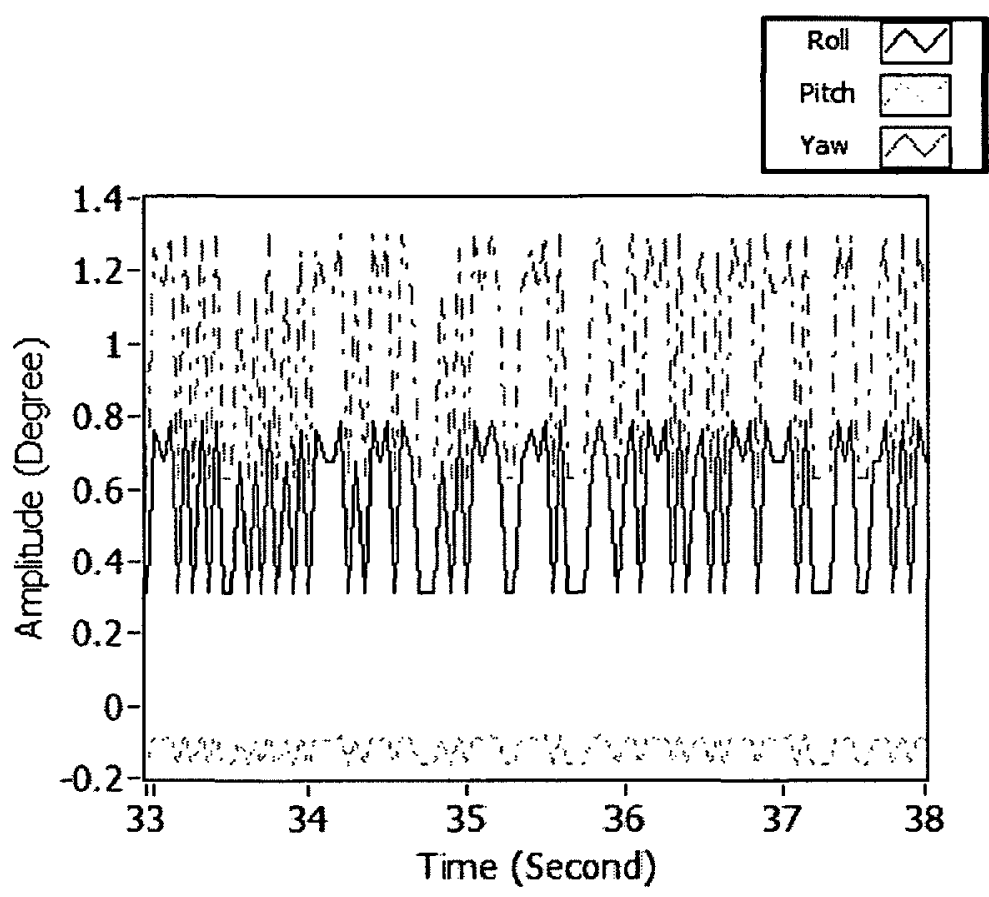

Figure 3.15: Illustration of the small errors of VOS readings.

error of less than $2^{\circ}$. The maximum error is large because there is a sharp turn in the simulation and the Atlas TDP could not follow the sudden change. Among most of the simulation experiments, the errors were in the range of $1^{\circ}$ to $3^{\circ}$. These tests confirm that with the newly developed VOS and the updated controller, the Atlas TDP can track the simulated aircraft trajectory successfully. The results are shown in Figure 3.16 and Table 3.6 .

\begin{tabular}{ccc}
\hline Axis & RMS Error & Maximum Error \\
\hline Yaw & $1.44^{\circ}$ & $5.34^{\circ}$ \\
Pitch & $1.87^{\circ}$ & $12.8^{\circ}$ \\
Roll & $1.32^{\circ}$ & $4.79^{\circ}$ \\
\hline
\end{tabular}

Table 3.6: RMS error and maximum error of a flight simulation 


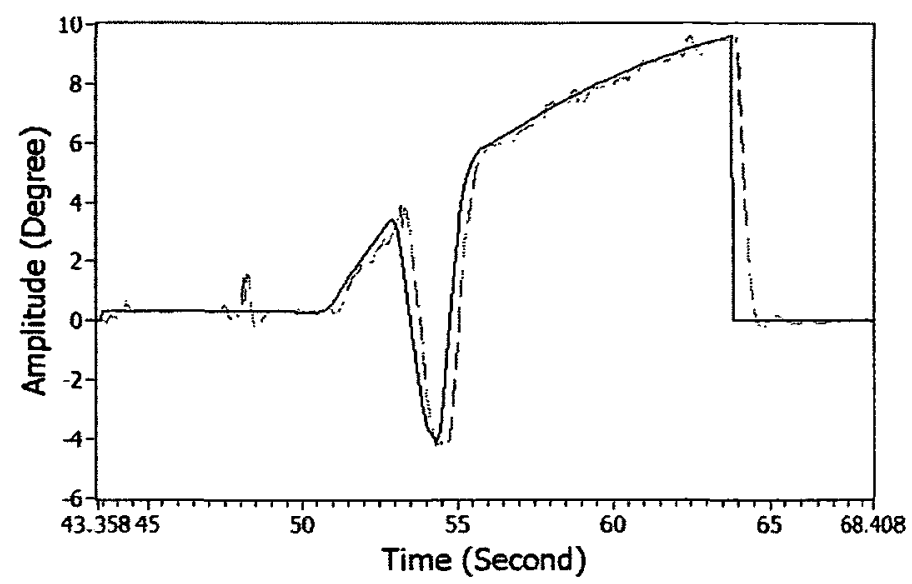

(a) Yaw

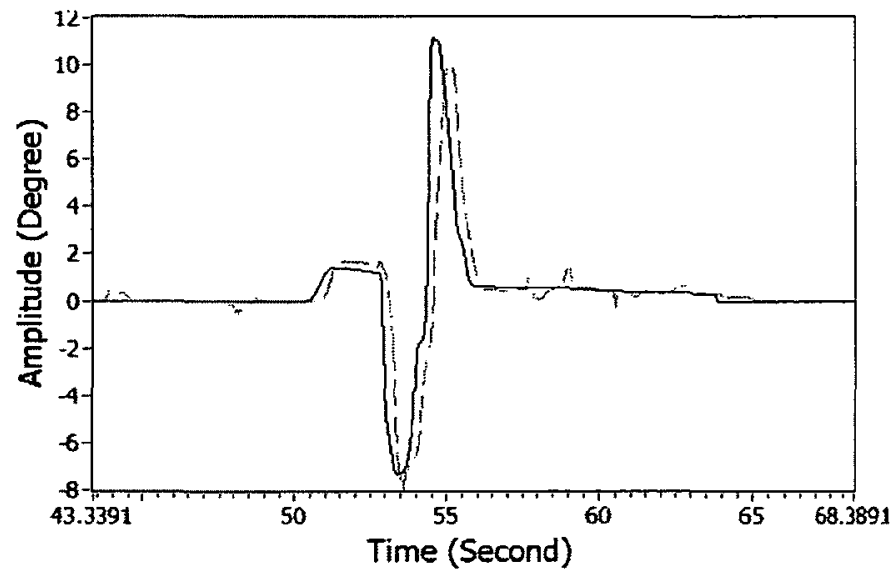

(b) Pitch

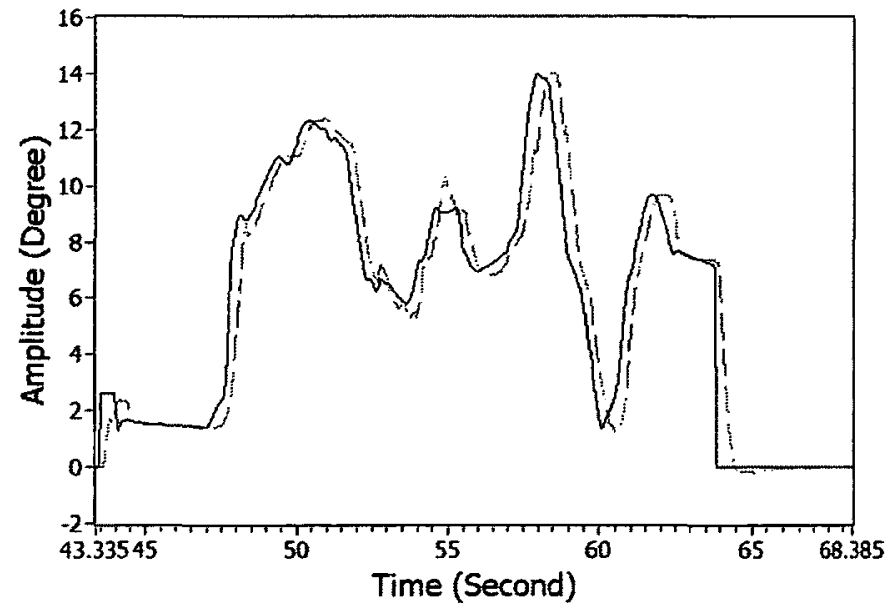

(c) Roll

Figure 3.16: Response of a Flight Simulation. 


\subsection{Discussion and Conclusion}

From all the experimental results stated above, it is observed that with VOS feedback, quaternion-based kinematic nonlinear controller (with an RMS error of less than $1^{\circ}$ in most circumstances) would meet the requirements of controlling the Atlas TDP. However, the performance of the system still contains slight vibrations, oscillations, and the stick-slip behaviour mainly caused by the actuation and sensing systems. The feedback from the VOS contains errors mainly because of the system delay, low sample speed, noise, and sphere structural imperfections. On the other hand, the sphere will not achieve the desired position from the controller because of the friction, imbalance of the three motors, dislocation of the center of gravity of the sphere, and misalignment of the sphere axes. All these factors contribute to imprecision of the system. The performance of this controller can be improved on a more carefully manufactured and assembled platform. The use of the VOS as the orientation feedback mechanism is successful as the VOS supplies the controller with an absolute measurement of the sphere orientation. It has the benefit of not accumulating error over time compared to an inertial sensor. The only drawback of the VOS is its long processing time limited by the frame rate of the camera.

In the next chapter, sensor fusion is introduced to fuse the data of the VOS and the IOS, so that the feedback rate is increased. The sensor fusion also provides a backup plan in case the VOS collapses in certain conditions, such as when a marker is damaged. 


\section{Chapter 4}

\section{Preliminary Sensor Fusion of VOS and IOS}

As shown previously, the VOS sensor can control the Atlas TDP independently and it achieves good performance. However, it is desired to produce reliable orientation measurements in the case of lower performance or reduction in the data quality of the VOS system. There are two main concerns: first, a need for backing up the VOS in the case of misidentification of markers; and second, improving the sample rate of the controller. In addition, the VOS might have the blur problem on images when the angular velocity of the sphere is large. Alternatively, the IOS may contain the error that has accumulated over time caused by drift. To solve these problems, a sensor fusion algorithm such as Kalman filtering is used to obtain an improved measurement estimate from the two sensor (IOS and VOS). Fusing the VOS and IOS sensors would provide a sensing approach that is more robust than either one individually.

This chapter introduces the application of a modified Unscented Kalman Filter (UKF) on the Atlas TDP for the orientation estimation. This UKF was simulated for the non-realtime Atlas Lite model and proved capable of enhancing the stability and accuracy of the simulator orientation. As stated previously, the VOS provides an absolute and more accurate measurement but is limited by speed; and the IOS responds well to fast inputs but suffers from drift. Therefore, the estimation of the orientation of the sphere is based on both the VOS and IOS measurements. The 
description of the filtering algorithm, its implementation and real-time test results are presented in this chapter.

\subsection{Unscented Kalman Filter}

A Kalman filter is an algorithm for the prediction and estimation of a discrete-time variable in a linear state-space model as a result of the pioneering work by Kalman in 1960 [40]. The simple Kalman filter cannot fuse the data in the case of Atlas because of its highly nonlinear dynamics (which does not yet have an accurate mathematical model). The Extended Kalman filter was developed and widely used for nonlinear systems; however, the EKF is not available for Taylor Series expansions for linearization when the system is not well known (as with the Atlas TDP) because no dynamic model is currently available. Instead, the UKF which uses the unscented transformation to solve the nonlinearity was developed for nonlinear system and is implemented as the fusion algorithm for Atlas. This algorithm includes a process model and a measurement model of the system. For Atlas, the process model involves the orientation data from the IOS and the measurement model is simply the VOS [29].

\subsubsection{Process Model}

The state vector on the Atlas platform is represented by the unit quaternion $q$ in vector notation introduced previously. A simple process model would then be a function of $q_{k}$ and $w_{k}$ such that,

$$
q_{k+1}=F\left(q_{k}, w_{k}\right)
$$


where the subscript $k$ denotes the time $k T_{s}\left(T_{s}\right.$ is the sampling period) [29], $w_{k}$ denotes the process noise at the time step $k$. The orientation of the sphere can be updated by integrating:

$$
\dot{q}=\frac{1}{2} q \cdot\left\{\begin{array}{l}
0 \\
\Omega
\end{array}\right\}
$$

where $\Omega$ is the angular velocity of the sphere. The discrete-time integration of this equation is given by [29]

$$
q_{k+1}=q_{k} \cdot r_{k}
$$

where $r_{k}=\left[\begin{array}{ll}a_{c} & a_{s} \theta\end{array}\right]^{T}, \theta=\Omega_{k} T_{s},\|\theta\|$ denotes the norm of $\theta, a_{c}=\cos (\|\theta\| / 2)$, and $a_{s}=\sin (\|\theta\| / 2) /\|\theta\|$. These equations convert the axis-angle $\theta$ to a unit quaternion. This process assumes the angular velocity vector remains constant over the integration time step $T_{s}$. The "." denotes a quaternion multiplication as in Equation 1.6. Note that the noise from the IOS measurement $V_{\omega}$ needs to be added to the angular velocity vector $\Omega$ of the sphere. Then the process function is generalized by

$$
q_{k+1}=F\left(q_{k}, \Omega+V_{\omega}, T_{s}, w_{k}\right)
$$

The angular velocity noise $V_{\omega}=n_{b}+n_{w}$, where $n_{b}$ denotes the bias of the gyro and is assumed to be a normally distributed random variable $n_{b} \sim \mathcal{N}\left(0, b_{\omega}^{2}\right)$ for the purpose of its application in a Kalman filter and $n_{\omega}$ denotes the gyroscope noise and is also assumed to be a normally distributed random variable $n_{\omega} \sim \mathcal{N}\left(0, \sigma_{\omega}^{2}\right)$. Both of the values of the bias and the gyro noise are given in the real-time implementation of the UKF. Therefore, the assumed IOS measurement noise is a normally distributed random variable $V_{\omega} \sim \mathcal{N}\left(0, Q_{\omega}\right)$, and $Q_{\omega}$ can be calculated from,

$$
Q_{w}=\left(\sigma_{\omega}^{2}+b_{\omega}^{2}\right) I_{3 \times 3}
$$


where $I_{3 \times 3}$ is a $3 \times 3$ identity matrix.

Although the state vector is a four-element vector, it depicts only three rotational DOF (orientation). Similar to the process described in [28], the state noise $w_{k}$ is represented in axis-angle format for orientation instead of quaternion format so that the noise better associates with the three rotational DOF. The process noise is defined as a $3 \times 1$ normally-distributed random variable $w_{k} \sim \mathcal{N}(0, P)$, where the covariance matrix $P$ is a $3 \times 3$ matrix [29]. The initial process noise is assumed to be:

$$
P_{0}=\left(\sigma_{\omega} T_{s}\right)^{2} I_{3 \times 3}
$$

An axis-angle vector $\vec{a}$ is converted to a unit quaternion $q$ by,

$$
q=\left\{\begin{array}{c}
\cos \left(\frac{\alpha}{2}\right) \\
\hat{a} \sin \left(\frac{\alpha}{2}\right)
\end{array}\right\}
$$

where $\alpha=\|\vec{a}\|, \hat{a}=\frac{\vec{a}}{\|\vec{a}\|}$. Also, the conversion from $q$ to $\vec{a}$ is given by,

$$
\vec{a}=\frac{2 \cos ^{-1}\left(q_{0}\right)}{\sqrt{1-q_{0}^{2}}}\left\{\begin{array}{l}
q_{1} \\
q_{2} \\
q_{3}
\end{array}\right\}
$$

The axis-angle process noise $w_{k}$ is then converted to a unit quaternion by substituting itself as $\vec{a}$ into Equation 4.7 and a new $q_{w}$ denoting the quaternion of the process noise is obtained. The process model is then finalized as

$$
q_{k+1}=\left(q_{k} \cdot r_{k} \cdot q_{w}\right)
$$




\subsubsection{Measurement Model}

The output vector of the measurement model is simply the orientation quaternion which is the same as the state vector:

$$
Z_{k}=q_{k}
$$

The process of VOS is a function of $n$ input markers's locations in the image frame ${ }^{i m} \mathrm{P}_{i}(i=1,2,3, \ldots, n)$ and their corresponding sphere frame locations ${ }^{s} \mathrm{P}_{i}(i=$ $1,2,3, \ldots, n)$ to produce the orientation of the sphere (as described in Subsection 2.3.2),

$$
Z_{k}=\operatorname{VOS}\left(P_{m}\right), \quad P m=\left\{\begin{array}{c}
{ }^{i m} \mathrm{P}_{i} \\
{ }^{s} \mathrm{P}_{i}
\end{array}\right\}
$$

The covariance matrix associated with the VOS measurement is defined in Equation 4.12 [29], where $\sigma_{i m}^{2}$ and $\sigma_{s}^{2}$ denote the covariance of the marker location measurement in the image frame and the sphere frame, respectively. Their values are known from camera calibration results and the manufacturing tolerances for the marker placement

$$
R_{m}=\left[\begin{array}{cc}
\sigma_{i m}^{2} I_{2 n \times 2 n} & 0_{2 n \times 3 n} \\
0_{3 n \times 2 n} & \sigma_{s}^{2} I_{3 n \times 3 n}
\end{array}\right]
$$

As described in Chapter 2, the VOS updates at a slower rate than the IOS. Therefore in the correction step, the orientation measurement of the VOS is not from the same step $k$ as of the IOS state but a previous step $l$. A technique called "out of sequence handling" $[29,41]$ used to deal with multi-rate sensor data incorporates a multi-rate hold that extrapolates from the historical values with interpolations or function approximations to determine the current measurement and state at the fastest sample 
rate. This technique is applied to calculate the updated measurement $Z_{k}^{\star}$ and the covariance $R$ at time step $k$ using Equation 4.13. Figure 4.1 illustrates the concept of the out of sequence handling. In the diagram, the system state is shown on the top line and the filter state is shown on the bottom line, with the time indicated along the $\mathrm{x}$-axis. The IOS data is passed to the filter at a high frequency, while the VOS measurement is delayed by $(k-l) T_{s}$ seconds. The VOS measurement at step $k$ is estimated by applying a rotation from step $l$ to step $k\left(q_{r o t}\right)$ to the VOS measurement at step $l$, as in Equation 4.13 [42], posteriori

$$
\begin{gathered}
q_{r o t}=\hat{q}_{k}^{-} \cdot\left(\hat{q_{l}^{+}}\right)^{-1} \\
Z_{k}^{\star}=q_{r o t} \cdot Z_{l}
\end{gathered}
$$

where $\hat{q}_{k}^{-}$denotes the a priori estimate of the state at step $k, \hat{q}_{l}^{+}$denotes the a posteriori estimation of orientation at step $l$, and $Z_{k}^{\star}$ represents the modified VOS measurement at step $k$. Note that $\left(\hat{q_{l}^{+}}\right)^{-1}$ is the inverse of $\hat{q}_{l}^{+}$in which the inverse quaternion is defined by

$$
q^{-1}=\frac{q_{0}+i q_{1}+j q_{2}+k q_{3}}{q_{0}^{2}+q_{1}^{2}+q_{2}^{2}+q_{3}^{2}}
$$

\subsubsection{UKF Implementation Structure}

UKF is a two-step estimation process generating "optimal" Kalman gains for nonlinear processes by propagating a number of test points (sigma points) through the state model and the measurement model to find the estimation state (a priori) and the corrected state estimate (a posteriori) [27]. 


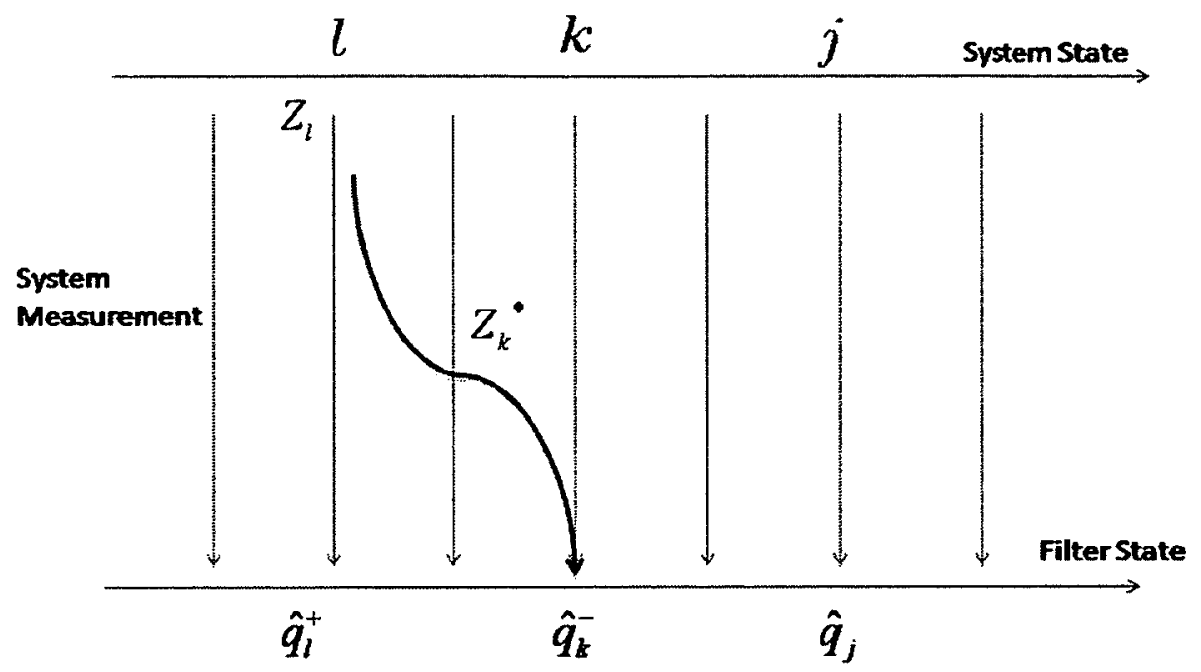

Figure 4.1: The out of sequence handling of VOS data

\section{A Priori}

The first step with the UKF is to obtain the a priori by prorogating the sigma points through the process model (Unscented Transformation). The set $X_{P}$ containing the sigma points of the disturbances from $w_{k}$ in axis-angle format are generated from the covariance estimate of the previous time-step $P_{k-1}^{+}$by $[28,29]$

$$
\begin{gathered}
\tilde{a}^{(i)}=\left[\sqrt{P_{k-1}^{+}}\right]_{i} i=1,2,3 \\
\tilde{a}^{(i+3)}=\left[\sqrt{P_{k-1}^{+}}\right]_{i} i=1,2,3
\end{gathered}
$$

where $\sqrt{P_{k-1}^{+}}$denotes the "square root" of $P_{k-1}^{+}$, $\tilde{a}$ denotes the sigma point in axis-angle format, and $i$ is the index. Note that the square root of $P_{k-1}^{+}$can be calculated by a Cholesky Decomposition as $P_{k-1}^{+}$is symmetric and positive definite. These $\tilde{a}^{(i)}$ are then converted to the quaternion representation $\hat{q}_{k-1}^{(i)}$ using Equation 4.7. The sigma points $\hat{q}_{k}^{(i)}$ are obtained by passing the $\hat{q}_{k-1}^{(i)}$ through the process model as Equation 4.9. 


\section{Calculating the Mean Orientation and Covariance}

The mean orientation $\bar{q}_{k}\left(\bar{Z}_{k}\right)$ is solved iteratively. The rotation between the sigma points and the mean are found, converted to axis-angle format, and then tested to see if their mean magnitude is sufficiently close to zero. The error rotation is calculated from

$$
e_{q}^{i}=\hat{q}_{k}^{(i)} \cdot\left(\bar{q}_{k}\right)^{-1}
$$

where $\hat{q}_{k}^{(i)}$ denotes the sigma points in $X_{p}$. This error rotation $e_{q}^{i}$ in quaternion format is then converted to the axis-angle format $e_{a}^{i}$ using Equation 4.8.

The initial mean orientation $\bar{q}_{k}$ is given by

$$
\bar{q}_{k}=\frac{1}{2 n} \sum_{i=1}^{2 n} \hat{q}_{k}^{(i)}
$$

The mean error rotation $\bar{e}_{a}$ is then calculated from

$$
\bar{e}_{a}=\frac{1}{2 n} \sum_{i=1}^{2 n} \hat{e}_{a}^{(i)}
$$

This mean error rotation is tested to see if its magnitude is sufficiently close to zero. If not, the mean error rotation calculated from Equation 4.18 is converted to quaternion format using Equation 4.7 and applied to Equation 4.19 to get the next $\bar{q}_{k}$

$$
\bar{q}_{k_{t+1}}=\bar{e}_{q} \cdot \bar{q}_{k_{t}} \quad \text { or } \quad \bar{Z}_{k_{t+1}}=\bar{e}_{q} \cdot \bar{Z}_{k_{t}}
$$

where $t$ denotes the index of iteration. The process is repeated from Equation 4.16. The a priori estimate mean $\hat{q}_{k}^{-}$and covariance $P_{k}^{-}$can now be determined from the 
mean and error rotations using the following three equations:

$$
\begin{gathered}
\hat{q}_{k}^{-}=\bar{q}_{k} \text { or } \hat{Z}_{k}^{-}=\bar{Z}_{k} \\
P_{q}=\frac{1}{2 n} \sum_{i=1}^{2 n}\left[e_{a}^{i}\right]\left[e_{a}^{i}\right]^{T} \\
P_{k}^{-}=P_{q}+Q_{i o s}
\end{gathered}
$$

The covariance of the IOS measurement noise $Q_{i o s}$ in Equation 4.20 is also determined by performing an unscented transformation through the process model using sigma points generated by the angular velocity noise $\omega_{m}$. The set $X_{Q}$ containing sigma. points of the angular velocity is generated by,

$$
\begin{gathered}
\omega_{m}^{(i)}=\omega_{m}+\tilde{\omega}^{(i)} \quad i=1,2, \ldots, 6 \\
\tilde{\omega}^{(i)}=\left[\sqrt{Q_{\omega}}\right]_{i} \quad i=1,2,3 \\
\tilde{\omega}^{(i+3)}=\left[\sqrt{Q_{\omega}}\right]_{i} \quad i=1,2,3 .
\end{gathered}
$$

These $\tilde{\omega}^{(i)}$ are passed through the process model (Equation 4.9) to get sigma points $\hat{q}_{k}^{(i)}$. The covariance matrix $Q$ is then calculated from,

$$
Q_{i o s}=\frac{1}{2 n} \sum_{i=1}^{2 n}\left[e_{a}^{i}\right]\left[e_{a}^{i}\right]^{T}
$$

where $e_{a}^{i}$ is the axis-angle format of $e_{q}^{i}$ calculated by Equation 4.16. The mean orientation $\bar{q}_{k}$ is also solved iteratively as described above. Note that both of $\hat{q}_{k}^{(i)}$ and $\bar{q}_{k}$ in calculating $Q_{i o s}$ are from the set $X_{Q}$. 


\section{Estimation of VOS Measurement}

The covariance matrix $R_{m}$ can now be used to create $10 n$ (the dimension of the VOS model is $5 n$ ) disturbances for the ${ }^{i m} \mathrm{P}_{i}$ and ${ }^{s} \mathrm{P}_{i}$ into the orientation calculation. Similar to the unscented transformation process as in Equations 4.15 and 4.21, $\tilde{P}_{m}^{(i)}$ are computed using $R_{m}$ so that the disturbances $P_{m}^{i}$ are obtained. They are then passed through the VOS measurement model (Equation 4.11) to calculate the sigma points of the measured orientation. Then the mean and covariance of the measured orientation $Z_{l}$ at time step $l$ are calculated,

$$
\begin{gathered}
Z_{l}=\bar{Z}_{l} \\
R_{v o s}=\frac{1}{2 n} \sum_{i=1}^{2 n}\left[e_{a}^{i}\right]\left[e_{a}^{i}\right]^{T}
\end{gathered}
$$

The quaternions and corresponding covariance matrix used to generate the sigma points are given as: $\left[\begin{array}{lll}Z_{l} q_{l}^{+} & q_{k}^{-}\end{array}\right]^{T} S=\left[\begin{array}{ccc}R_{v o s} & 0_{3 \times 3} & 0_{3 \times 3} \\ 0_{3 \times 3} & \hat{P}_{l}^{+} & 0_{3 \times 3} \\ 0_{3 \times 3} & 0_{3 \times 3} & \hat{P}_{k}^{-}\end{array}\right]$. The a priori estimate can be calculated from Equation 4.24 .

$$
\begin{gathered}
\hat{Z}_{k}=\hat{q}_{k}^{-} \\
P_{z}=P_{q}+R
\end{gathered}
$$

\section{A Posteriori}

The Kalman gains are computed from Equation 4.25.

$$
K_{k}=P_{q} P_{z}^{-1}
$$


The state is then updated by finding the innovation error rotation $V_{k}$ from,

$$
V_{k}=Z_{k}^{\star}-\hat{Z}_{k}
$$

where $Z_{k}^{\star}$ is from Equation 4.13. Applying the Kalman gains to $V_{k}$ and adding to the a priori state will yield the a posteriori state estimate mean $\hat{q}_{k}^{+}$and covariance $P_{k}^{+}$,

$$
\begin{gathered}
\hat{q}_{k}^{+}=\hat{q}_{k}^{-}+K_{k} V_{k} \\
P_{k}^{+}=P_{k}^{-}-K_{k} P_{z} K_{k}^{T}
\end{gathered}
$$

Figure 4.2 shows the flow chart of the UKF.

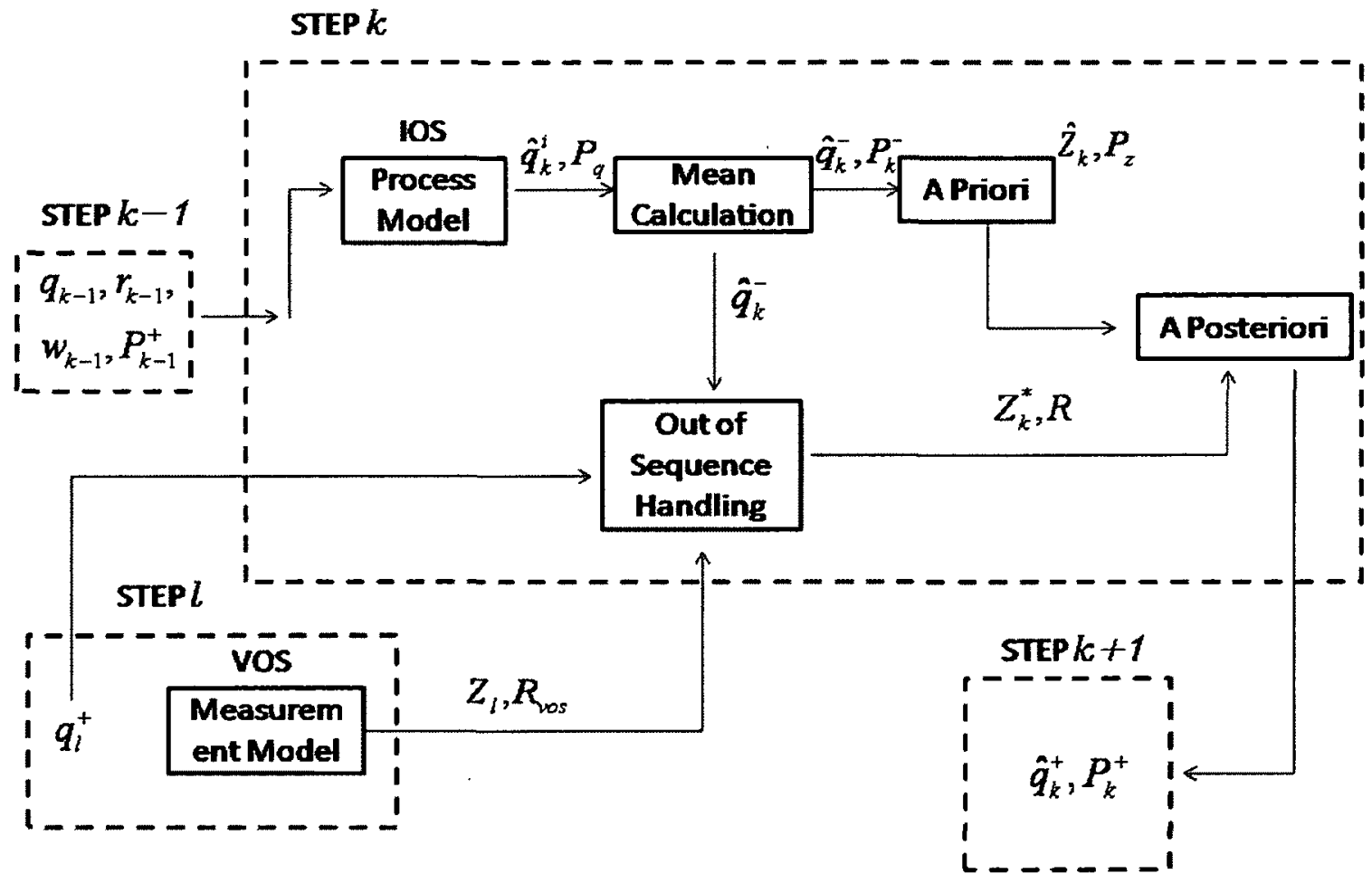

Figure 4.2: The flow chart of the UKF. 


\subsection{Real-time Implementation}

The real-time implementation was conducted using the LABVIEW real-time module. The marker standard deviations are set as $\sigma_{i m}=0.5$ pixels and $\sigma_{s}=5 \mathrm{~mm}$ (see Chapter 2). As introduced in Chapter 1, the IOS has characteristics listed in Table 4.1 [29]

Table 4.1: 3DM-GX1 Specifications

\begin{tabular}{ccc}
\hline Parameter & Value & Units \\
\hline Dynamic range & \pm 300 & $\% / \mathrm{s}$ \\
Angular random walk $\left(N_{A R W}\right)$ & 3.5 & $\% \sqrt{\mathrm{hr}}$ \\
Bias $\left(b_{\omega}\right)$ & 0.1 & $\% / \mathrm{s}$ \\
\hline
\end{tabular}

The dynamic range of the triaxial gyroscopes describes the maximum angular velocity of each gyroscope. The angular random walk describes the noise of the gyroscope, which is used to define the normally-distributed random variable $n_{\omega} \sim \mathcal{N}\left(0, \sigma_{\omega}^{2}\right)$, where the standard deviation $\sigma_{\omega}$ is calculated using

$$
\sigma_{\omega}=\frac{N_{A R W}}{T_{s}}
$$

where $N_{A R W}$ is the angular random walk noise of the gyroscope and $T_{s}$ is the sampling period [29]. The bias $b_{\omega}$ is the maximum uncompensated bias of the gyroscope sensor that causes the drift. In the real-time implementation, the two variables were calculated as $\sigma_{\omega}=0.00852 \mathrm{rad} / \mathrm{s}$ and $b_{\omega}=0.1 \mathrm{deg} / \mathrm{s}$.

The sample rate of the IOS was selected to be $70 \mathrm{~Hz}$ while the sample rate of the VOS is $18 \mathrm{~Hz}$. Therefore, these two models are separated into two timed loops in LABVIEW. Figure 4.3 shows the block diagram of the structure of the sensor fusion program named as VOS_IOS_fusion.vi. 


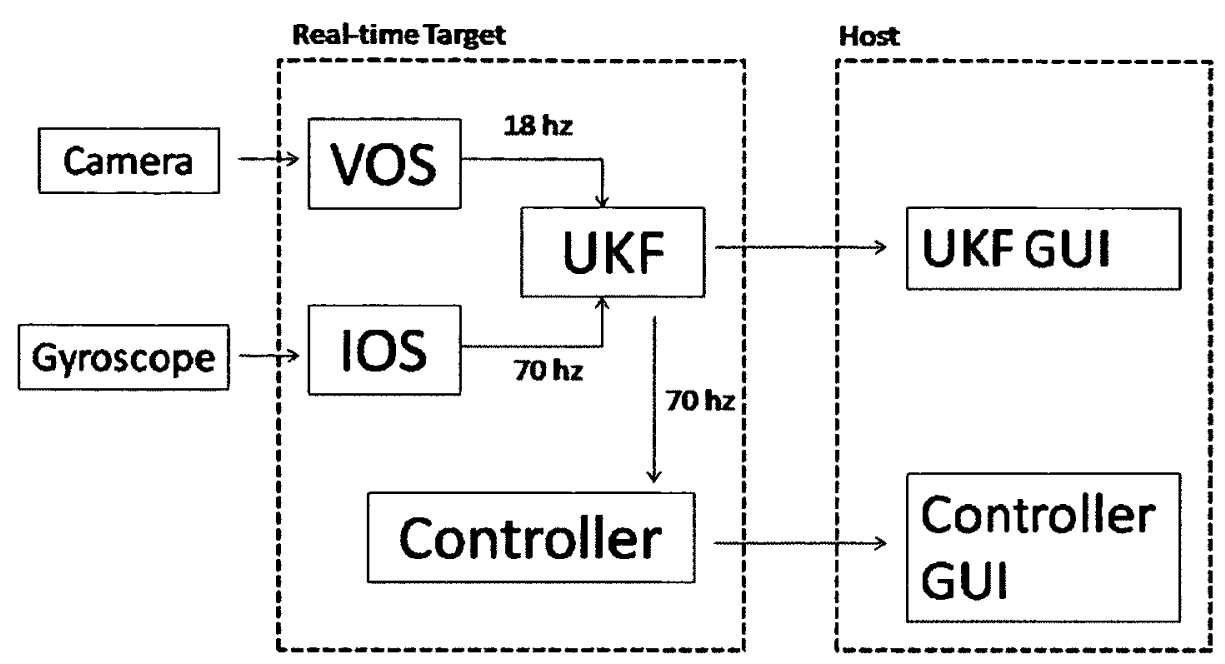

Figure 4.3: The block diagram of the sensor fusion incorporating with the controller

\subsubsection{Test Results}

To verify the performance of the sensor fusion algorithm with the UKF, three groups of tests were performed on three aspects on the Atlas TDP. Note that in all of the figures below, the fused data was denoted with the black solid line; the VOS data was denoted with the grey dotted line; the IOS data was denoted with the dashed light grey line.

The sequence of the three groups of tests were as follows.

1. First, verify the robustness of the fused data against the case where VOS and IOS fail with single-DOF motion when the IOS errors were small.

2. Second, similar to the first but conducted in multi-DOF when the IOS errors were large.

3. Third, verify the increase of the sample rate as well as the improvement in the orientation control performance. 


\section{Test 1}

In Test 1 , the TDP sphere was doing a single DOF motion which was rotating about the roll, pitch, and yaw axes respectively at certain angular velocities $(0.2 \mathrm{rad} / \mathrm{s}$ for roll and pitch, $0.1 \mathrm{rad} / \mathrm{s}$ for yaw) in turn. To verify the performance of the fused orientation data, artificial disturbances were planted in the VOS data during the test by simply covering the markers for a short time so that the algorithm produced a "not a number (NAN)" (from $17.5 \mathrm{~s}$ to $18.5 \mathrm{~s}$ in Figure 4.5 and at $27 \mathrm{~s}$ in Figure 4.6). The IOS error was not planted artificially but came from the sensor itself . It is important to note that before Test 1 , the system was stable and not much error was accumulated in the IOS. Therefore, the errors between the IOS and VOS data were not large (refer to Figures 4.4 and 4.6 ).

Figures 4.4, 4.5 and 4.6 show the comparisons between the quaternion orientations obtained using VOS, IOS, and fused data. In each figure, only one component of the vector part of the quaternion corresponding to the rotation axis is displayed since the rotation is single DOF.

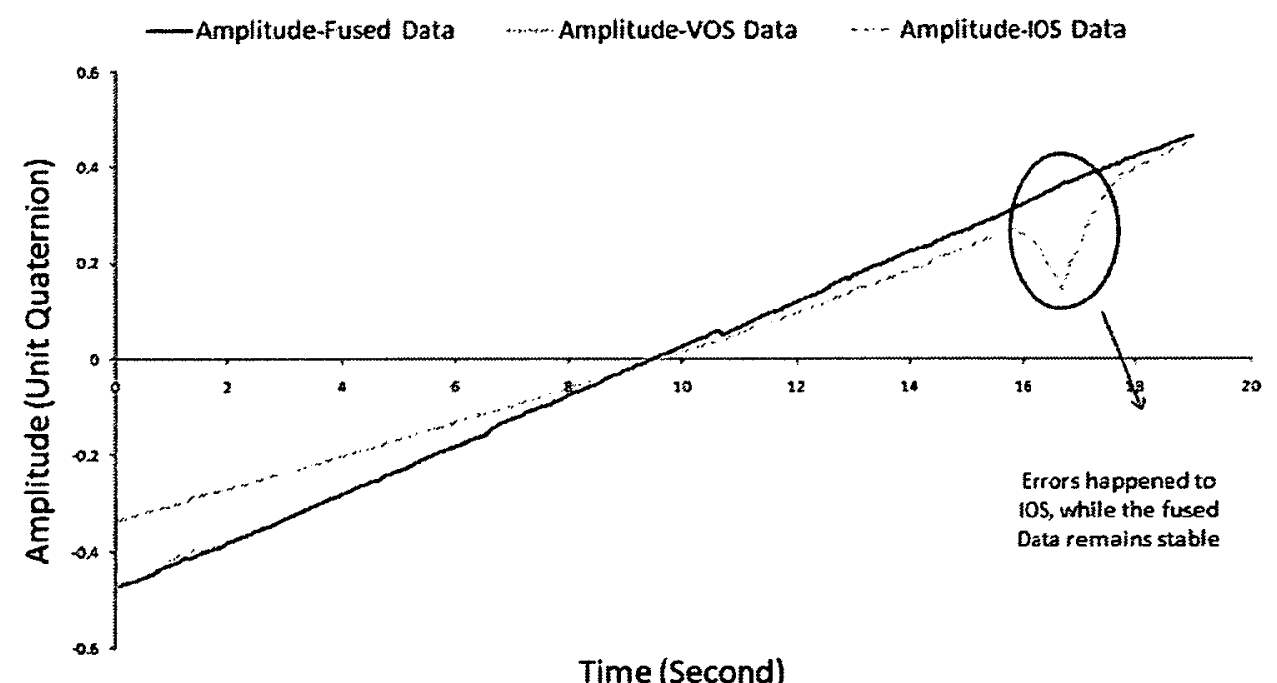

Figure 4.4: The VOS, IOS, and fused data about the pitch axis in Test 1. 


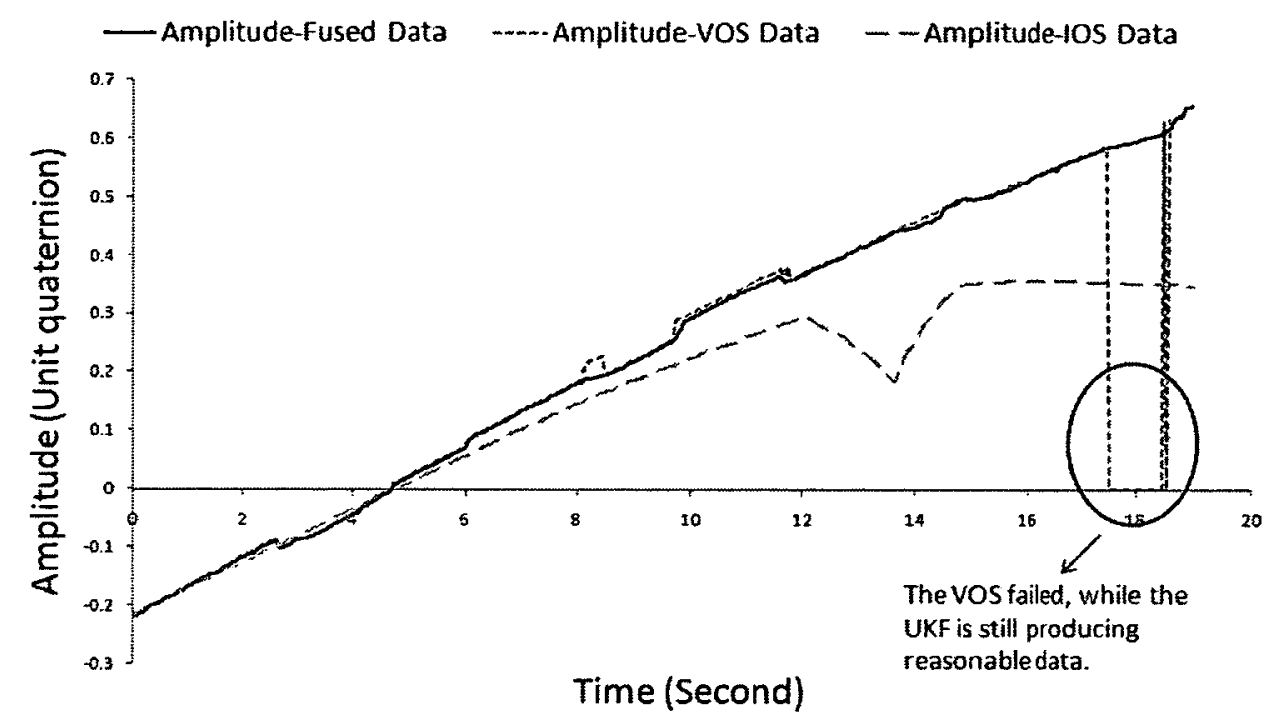

Figure 4.5: The VOS, IOS, and fused data about roll axis in Test 1.

Since the real orientation data was unknown (the VOS orientation data is assumed to be the closest one to the real orientation), the errors between the real and measured orientation are not computed here. As indicated by the circle in Figure 4.4, it was observed that when the pitch component of $q$ is around 0.3 , the IOS data had a large error that might have been caused by various things such as the bias, external magnetic field, mechanical vibrations, etc., while the fused data remained close to the VOS data. The fused data corresponding to cases where the VOS fails is shown by the circles in Figures 4.5 and 4.6. However, the UKF can still produce reasonable orientation data based on predicting the rotation between the previous and current step using the angular velocity calculated from the IOS. This scheme would work for a short period of time when VOS data continues to be erroneous for a prolonged period. However, if the VOS data keeps missing, the IOS error would be introduced to the system by the angular velocity. 


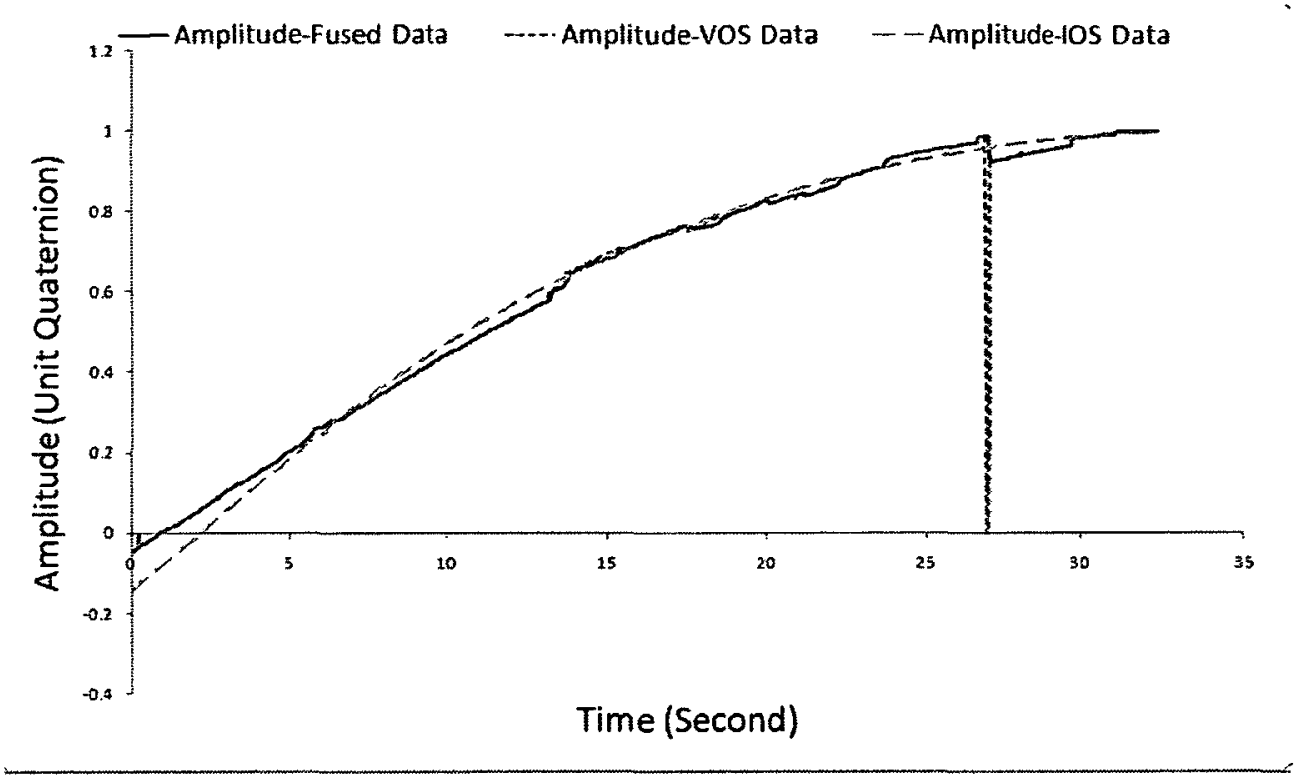

Figure 4.6: The VOS, IOS , and fused data about yaw axis in Test 1.

\section{Test 2}

In the test, the system was run for a long time priori to initiating Test 2. In Test 2 the TDP sphere was rotating about all three axes simultaneously at the angular velocity of $0.03 \mathrm{rad} / \mathrm{s}$ (in order to increase the IOS error). The VOS was producing "NAN" from $3.5 \mathrm{~s}$ to $4 \mathrm{~s}$. The results are shown in Figures 4.7, 4.8, and 4.9. An offset in the IOS data was observed due to the accumulated errors, while the fused data kept close to the VOS data. When the VOS failed again from $3.5 \mathrm{~s}$ to $4 \mathrm{~s}$, errors were introduced to the fused data from the IOS. 


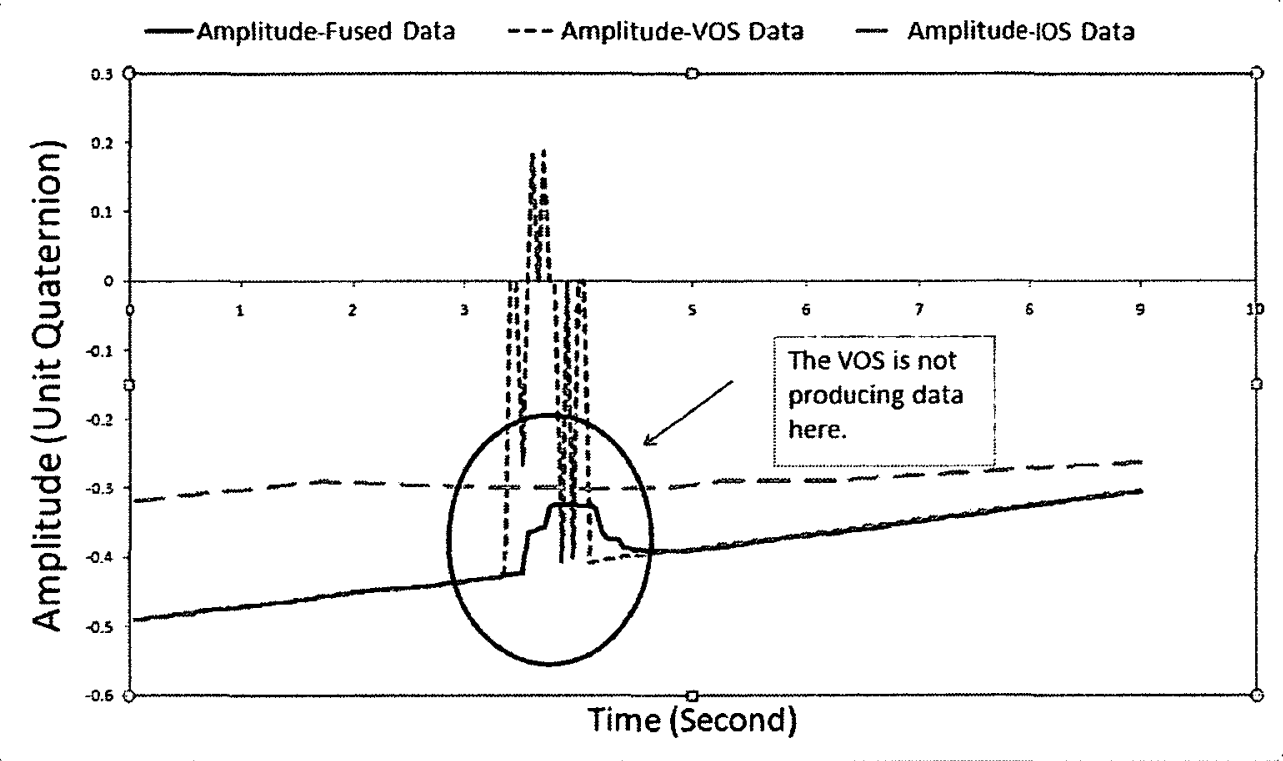

Figure 4.7: The VOS, IOS, and fused data about the pitch axis in Test 2.

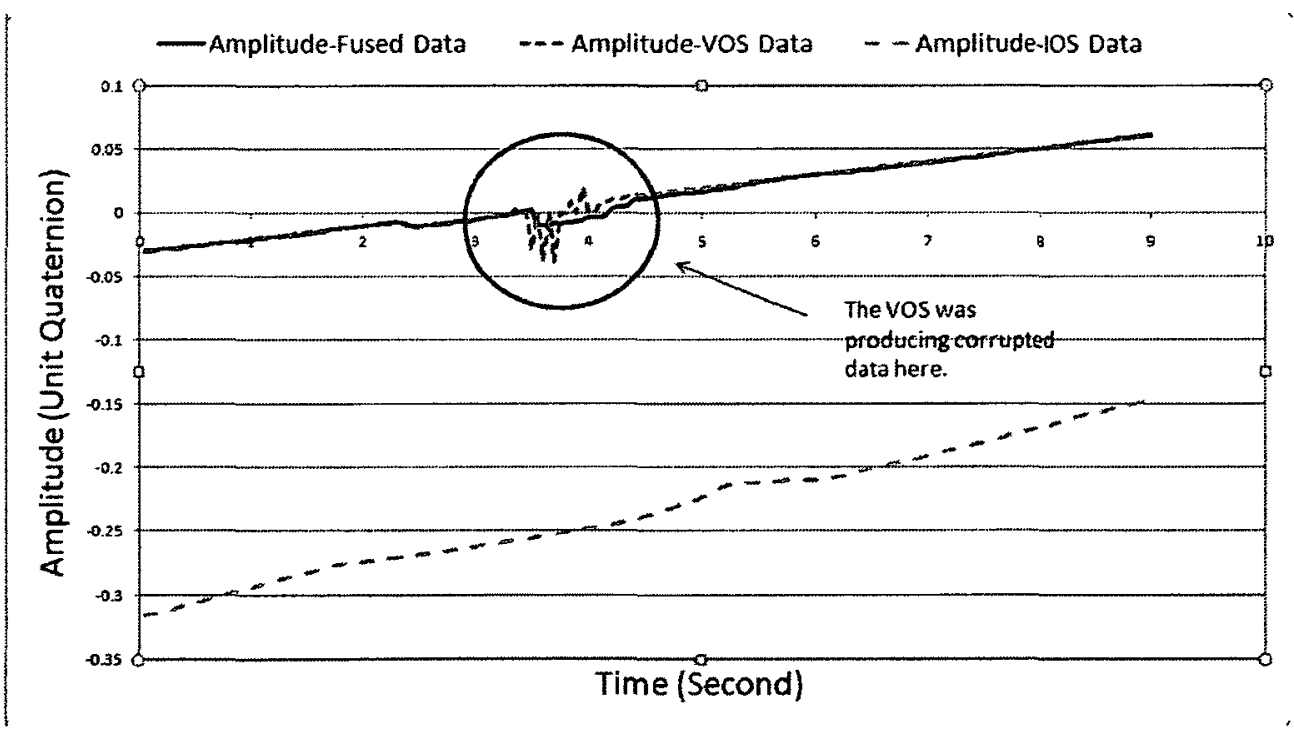

Figure 4.8: The VOS, IOS, and fused data about roll axis in Test 2. 


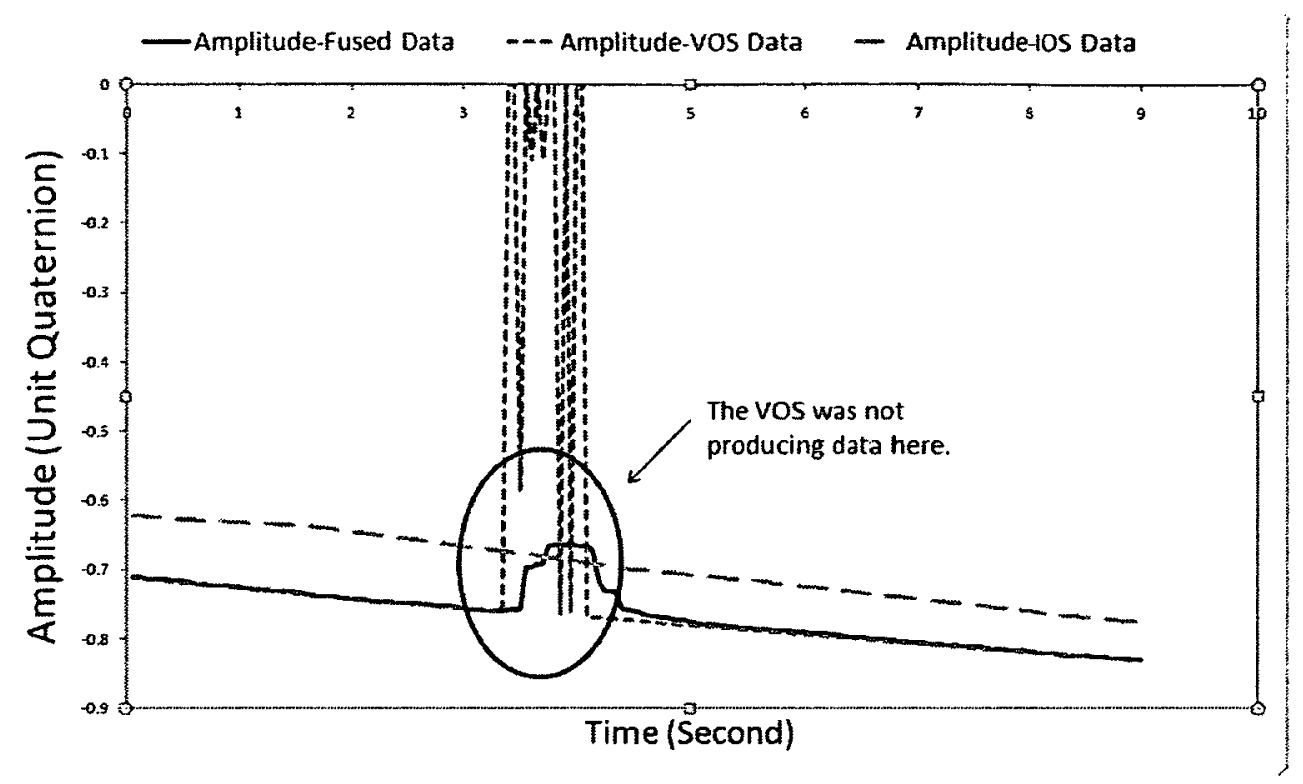

Figure 4.9: The VOS, IOS, and fused data about yaw axis in Test 2.

\section{Test 3}

In test 3 , the estimated VOS data (from out of sequence handling) which has higher sample rate than the unestimated VOS data (the raw orientating data computed by the VOS) was sent to the nonlinear controller. The system was asked to follow a multiDOF sinusoidal trajectory with an amplitude of $10^{\circ}$. Figure 4.10 shows the multi-DOF sinusoidal response of the nonlinear controller with the estimated VOS feedback which is faster and smoother than the simple VOS feedback. It is found that the sinusoidal tracking response of the TDP system had less oscillations at the transient point (top and bottom of the sinusoidal curve) than that when the feedback was the simple VOS feedback. 


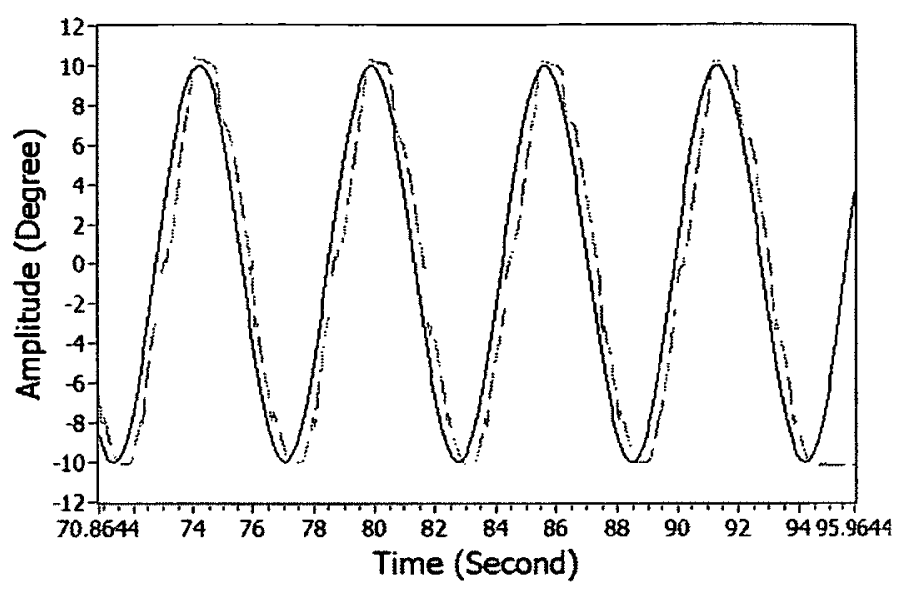

(a) Pitch

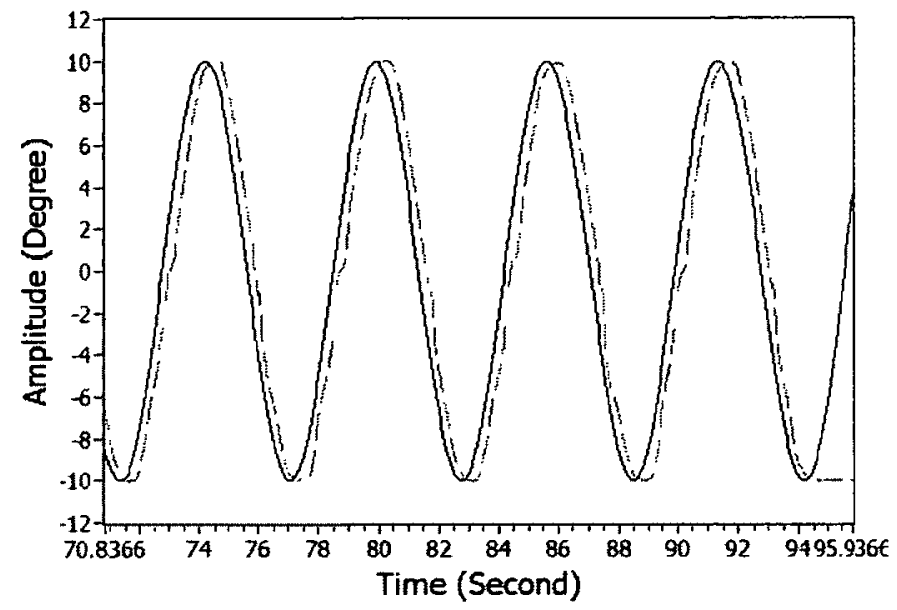

(b) Roll

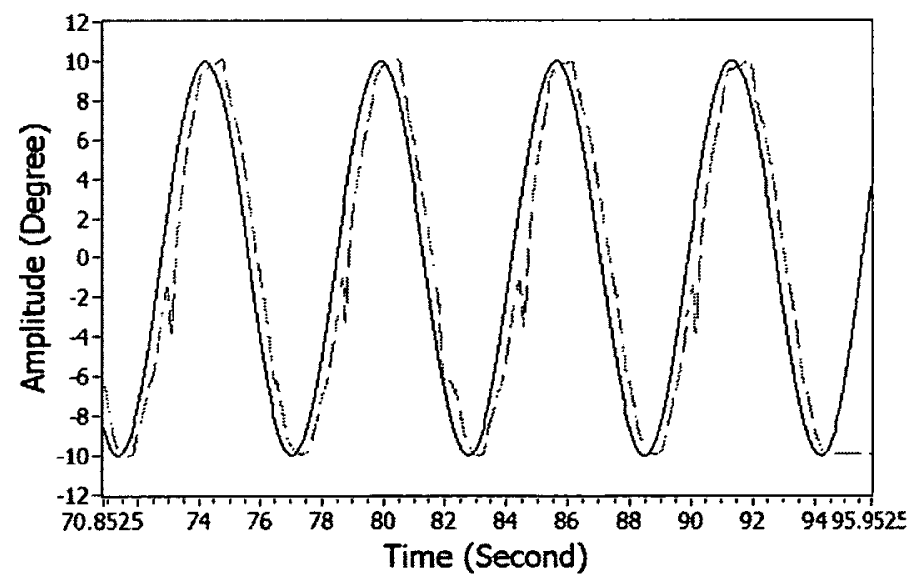

(c) Yaw

Figure 4.10: Response of a sinusoidal input of the controller in Test 3 


\subsection{Discussion and Conclusion}

From the results of tests 1 and 2, it was found that the implemented UKF can produce acceptable orientation data, regardless of the temporary failure of VOS and the IOS error. From the results of Test 3 , it was observed that the TDP controller worked better with the estimated VOS data than the single VOS data. The reason is that the estimated VOS feedback took the markers centre positions errors and marker placements into the estimation measurement model, so that the effects of these errors were minimized. The sample rate was also improved from $18 \mathrm{~Hz}$ to $70 \mathrm{~Hz}$ so that more data were fed into the controller.

Overall, an UKF algorithm using the unscented transformation to solve the nonlinearity of the system was developed and implemented on the Atlas TDP for the purpose of fusing the VOS and IOS orientation data. Several tests were conducted in real time to validate the performance of the sensor fusion algorithm. The filtered VOS data were sent to the controller and performed better than the unfiltered VOS data. From the results of all the tests, the UKF algorithm is approved to meet the requirements of: backing up the VOS in the case of failure and increasing its sample rate. 


\section{Chapter 5}

\section{Conclusions and Future Work}

\subsection{Conclusions}

\subsubsection{Vision-based Orientation Sensor}

A vision-based orientation sensor (VOS) was developed to provide an absolute measurement of the orientation of the Atlas TDP. This VOS design contains a novel design of digital-ID markers for positioning, camera calibration for building the mapping relation between the camera space and the $3 \mathrm{D}$ world space, and the orientation calculation algorithm. The VOS was implemented on a LABVIEW real-time operating system (RTOS) at a frequency of $18 \mathrm{~Hz}$. Several groups of tests for validating the accuracy of the VOS were conducted by rotating the sphere around yaw, pitch, and roll axes for $120^{\circ}$. The measurements of the inertial orientation sensor (IOS) and the VOS were recorded and compared. The test results showed that the VOS maintains accurate orientation measurement as having an average error of less than $1^{\circ}$, while the IOS was not stable and reliable as its average error was over $10^{\circ}$. These results indicate that the newly-developed VOS can measure the absolute orientation of the Atlas TDP with the only drawback of a slow update rate. 


\subsubsection{Vision-based Orientation Control}

A nonlinear kinematic controller using the orientation feedback from the VOS in quaternion representation was selected from the open literature and modified to control the Atlas TDP. This controller was implemented and validated on the Atlas TDP in real-time and improved through controller gain scheduling. The control law takes the desired angular velocity and orientation of the sphere as the inputs, the difference between the VOS measured orientation and the reference orientation as the error, and the command angular velocity of the sphere as the output. In the gain scheduling model, the proportional gain is defined as a function of the error. A kinematic model is applied to the angular velocity of the sphere to produce the angular velocity of the motors. This controller was implemented on the RTOS and tested with single-DOF and multi-DOF trajectories, as well as a real flight simulation trajectory. This controller had $0.01^{\circ}$ of steady state error and less than $0.7^{\circ}$ of RMS error regardless of the time shift of the mechanical system. These results showed that the controller is stable and capable of controlling the Atlas TDP.

\subsubsection{Sensor Fusion of VOS and IOS}

Sensor fusion of VOS and IOS using an unscented Kalman Filter (UKF) was developed on the Atlas TDP. This provides a powerful backup measurement system in case of VOS fails (such as when a marker is misidentified). The fusion method provides a more reliable measurement of the orientation than the VOS and IOS alone, and also improves the sample rate of the control system by generating estimates in between measured samples. The UKF was implemented and tested on the LABVIEW realtime module. From the results data, it was observed that:

1. The fusion algorithm was robust against the potential failures of the VOS or error 
accumulation of the IOS.

2. There were improvements in the smoothness and error levels of the controller response using the feedback from the estimated VOS.

3. A more robust and reliable control can be achieved using the fused data compared to using individual sensors.

\subsection{Future work}

A few directions of future work are recommended to enhance this research:

1. A more robust image processing technique can be developed to produce reliable marker position data in the case of faded or damaged markers. This algorithm can benefit from the redundancy of the information in the circular bar code patterns of the markers.

2. The marker distributions can be changed as the distinct markers are close to each other to reduce the error due to the adjacent similar markers.

3. The use of multiple cameras would result in lesser chances of losing sufficient markers for the orientation computation. Therefore, for the full-scale Atlas system, it is proposed to use multiple cameras for the orientation measurement.

4. The motor shaft encoders can be part of the sensor fusion algorithm. This not only provides three sources of measurement resulting in more reliable data, it can also be used as a means to estimate the wheel-sphere slippage. 


\section{List of References}

[1] A. K. Gary Bradski. Learning OpenCV. O'Reilly Media, Inc (2008 Sept).

[2] K. Klumper, A. Morbi, K. Chisholm, R. Beranek, M. Ahmadi, and R. Langlois. "Orientation control of atlas: A novel motion simulation platform." Mechatronics 22(8), 1112 - 1123. ISSN 0957-4158 (2012).

[3] W. Bles and E. Groen. "The desdemona motion facility: Applications for space research." Microgravity Science and Technology 21, 281-286. ISSN 0938-0108 (2009).

[4] J. Kim, J.-C. Hwang, J.-S. Kim, and F. Park. "Eclipse-ii: a new parallel mechanism enabling continuous 360-degree spinning plus three-axis translational motions." In "Robotics and Automation, 2001. Proceedings 2001 ICRA. IEEE International Conference on," volume 4, pages 3274 - 3279 vol.4. ISSN 1050-4729 (2001).

[5] P. Giordano, C. Masone, J. Tesch, M. Breidt, L. Pollini, and H. Bu?lthoff. "A novel framework for closed-loop robotic motion simulation - part i: Inverse kinematics design." In "Robotics and Automation (ICRA), 2010 IEEE International Conference on," pages 3876 -3883. ISSN 1050-4729 (2010).

[6] CUSP. "Final report year 2." CUSP Final Report (2003-2004).

[7] A. Weiss, R. Langlois, and M. Hayes. "The effects of dual row omnidirectional wheels on the kinematics of the atlas spherical motion platform." Mechanism and Machine Theory 44(2), 349 - 358. ISSN 0094-114X (2009).

[8] CUSP. "Final report year 4." CUSP Final Report (2005-2006).

[9] CUSP. "Final report year 5." CUSP Final Report (2006-2007).

[10] CUSP. "Final report year 9." CUSP Final Report (2010-2011). 
[11] J. Plumpton. "Atlas jacobians cheat sheet." TM-DYNjp.12.AtlasJacobians.03 (2010-2011).

[12] J. Plumpton. "Wheel selection and development of analytical tools for the atlas simulator." CUSP DESIGN REPORT DR-DYNjp.11.AtlasAnalysisToolsDevelopment.01 (2010-2011).

[13] MicroStrain, 310 Hurricane Lane, Unit 4. Williston, Vermont. Detailed Specifications For 3DM-GX1.

[14] J. Feng. "The study of $3 \mathrm{dm}-\mathrm{gx} 1$ ios sensor for the new design full scale sphere." CUSP DESIGN REPORT DR-CNT-jf.IOS (2009-2010).

[15] K. Chisholm. "Sphere orientation sensor." CUSP DESIGN REPORT DR-CNTkc.08.SOS.01.pdf (2007-2008).

[16] Z. Qian, Q. Wang, L. Ju, A. Wang, and J. Liu. "Studies on vision based absolute orientation detection method of spherical motor." In "Electrical Machines and Systems, 2009. ICEMS 2009. International Conference on," pages 1 -6 (2009).

[17] H. Garner, M. Klement, and K.-M. Lee. "Design and analysis of an absolute non-contact orientation sensor for wrist motion control." In "Advanced Intelligent Mechatronics, 2001. Proceedings. 2001 IEEE/ASME International Conference on," volume 1, pages $69-74$ vol.1 (2001).

[18] R. Neild. "Full scale implementation of the visual orientation sensor." CUSP DESIGN REPORT DR-CNT-rn.FullScaleVOS.01 (2010).

[19] I. Zafar, U. Zakir, and E. A. Edirisinghe. "Real-time multi-barcode reader for industrial applications." pages 772404-772404-9 (2010).

[20] M. Maghrabey. "Tdp sphere actuation control system design." CUSP DESIGN $R E P O R T \quad M o M .12 . T D P . S p h e r e . A c u t a t i o n . C o n t r o l . S y s t e m . D e s i g n .01$ (2011-2012).

[21] J. Spark. "Atlas control algorithm design." RTP.js.11.Atlas.Control.Algorithm.Design.01 (2010-2011).

[22] J. Yuan. "Closed-loop manipulator control using quaternion feedback." Robotics and Automation, IEEE Journal of 4(4), 434 -440. ISSN 0882-4967 (1988).

[23] S. Joshi, A. Kelkar, and J.-Y. Wen. "Robust attitude stabilization of spacecraft using nonlinear quaternion feedback." Automatic Control, IEEE Transactions on 40(10), $1800-1803$. ISSN 0018-9286 (1995). 
[24] J.-Y. Wen and K. Kreutz-Delgado. "The attitude control problem." Automatic Control, IEEE Transactions on 36(10), 1148 -1162. ISSN 0018-9286 (1991).

[25] CUSP. "Final report year 8." CUSP Final Reprt (2009-2010).

[26] J. Wang and W. Wilson. "3d relative position and orientation estimation using kalman filter for robot control." In "Robotics and Automation, 1992. Proceedings., 1992 IEEE International Conference on," pages 2638 -2645 vol.3 (1992).

[27] S. Julier and J. Uhlmann. "Unscented filtering and nonlinear estimation." Proceedings of the IEEE 92(3), 401 - 422. ISSN 0018-9219 (2004).

[28] E.Kraft. "A quaternion-based unscented kalman filter for orientation tracking." Proceeding Sixth International Conference of Information Fusion 1, 47-54 (2003).

[29] K. Chisholm. "Inertial measurement unit and vision fusion for a novel simulator motion platform." Direct Study Report (2009).

[30] J. Linseman. A UKF-basd orientation estimator of the Atlas platform. Master's thesis, Carleton Unversity (2010).

[31] A. Paterson. "Inertial orientation sensor using quaternions." $C U$ DR-SYSap.08.IOSwithQuaternions.02 (2007-2008).

[32] M. Fiala. "Artag, a fiducial marker system using digital techniques." In "Computer Vision and Pattern Recognition, 2005. CVPR 2005. IEEE Computer Society Conference on," volume 2, pages 590 - 596 vol. 2. ISSN 1063-6919 (2005).

[33] K. Klumper. "Vos marker scheme and rotational algorithm." CUSP DESIGN REPORT Dr-CNT-kk.10.VOSMarkerrotation.01 (2009-2010).

[34] NDI. "Optotrak certus motion capture system."

[35] R. Tsai. "A versatile camera calibration technique for high-accuracy $3 \mathrm{~d}$ machine vision metrology using off-the-shelf tv cameras and lenses." Robotics and Automation, IEEE Journal of 3(4), 323-344. ISSN 0882-4967 (1987).

[36] Z. Zhang. "Flexible camera calibration by viewing a plane from unknown orientations." In "Computer Vision, 1999. The Proceedings of the Seventh IEEE International Conference on," volume 1, pages 666 -673 vol.1 (1999).

[37] J.-Y. Bouguet. "Camera calibration toolbox for matlab." (2010). 
[38] D. Brown. "Close-range camera calibration." Photogrammetric Engineering 37, $855 \mathrm{C} 866$ (1971).

[39] J. Heikkila and O. Silven. "A four-step camera calibration procedure with implicit image correction." In "Computer Vision and Pattern Recognition, 1997. Proceedings., 1997 IEEE Computer Society Conference on," pages $1106-1112$. ISSN 1063-6919 (1997).

[40] R. . L. . Eubank. A Kalm Filter Primer. Chapman and Hall (2005).

[41] L. Armesto, J. Tornero, and M. Vincze. "Fast ego-motion estimation with multirate fusion of inertial and vision." The International Journal of Robotics Research 26(6), 577-589 (2007).

[42] L. A. J. T. M. Vincze. "Fast ego-motion estimation with multi-rate fusion of inertial and vision." The International Journal of Robotics Research 26(6), 577589 (2007). 
Appendix A 


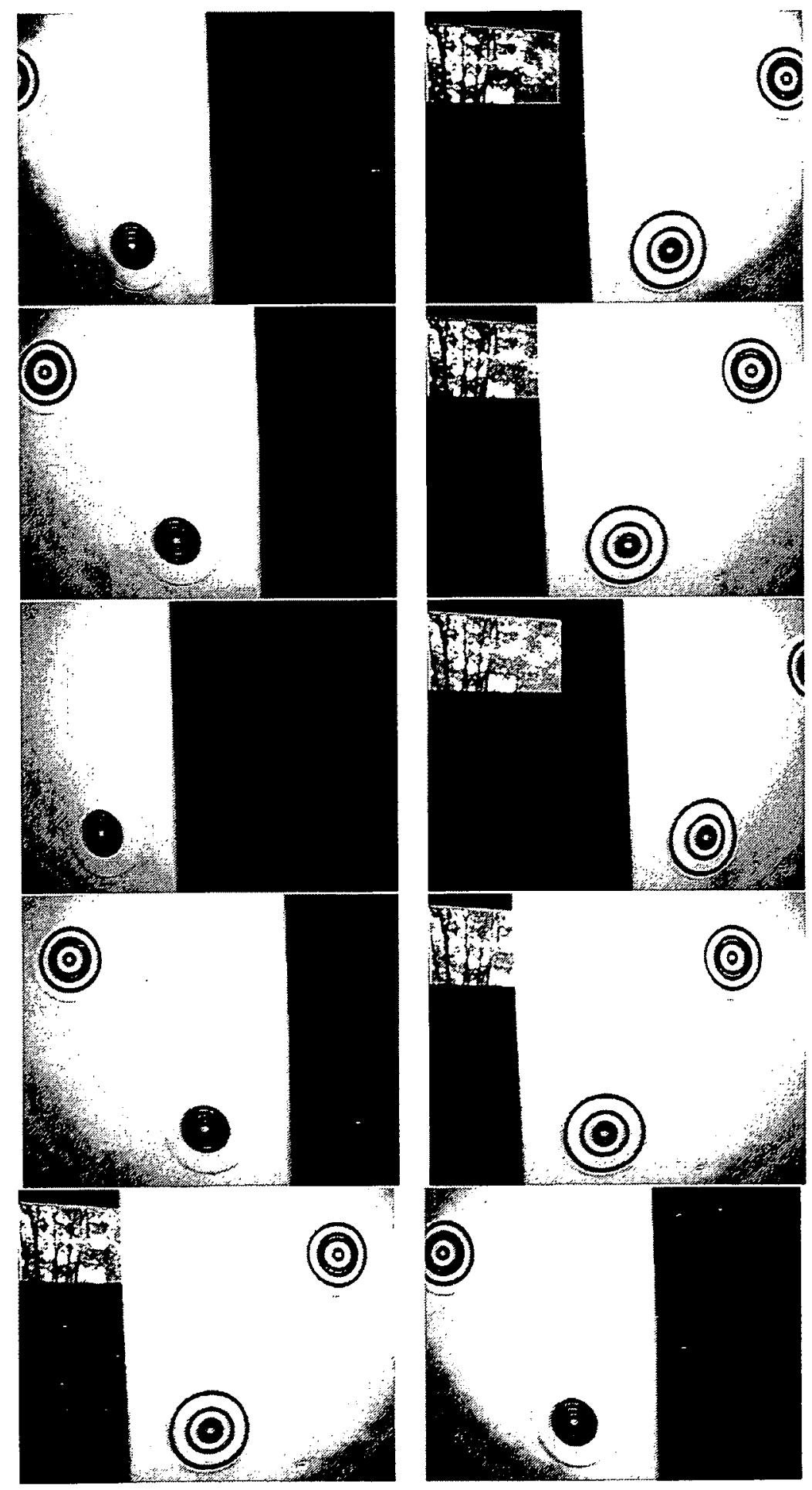




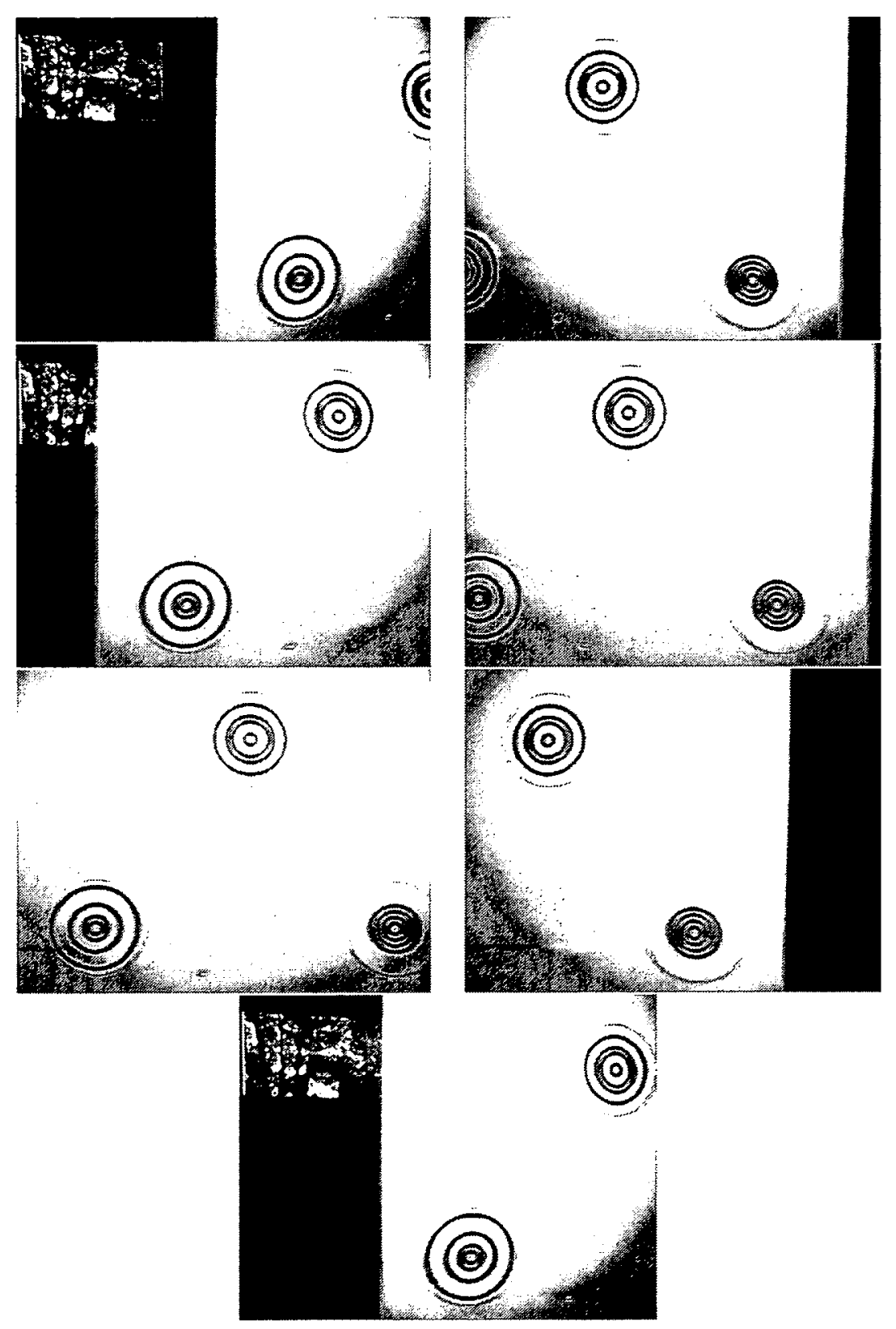

Figure A.0: 6 and $4 \mathrm{~cm}$ markers on white background 


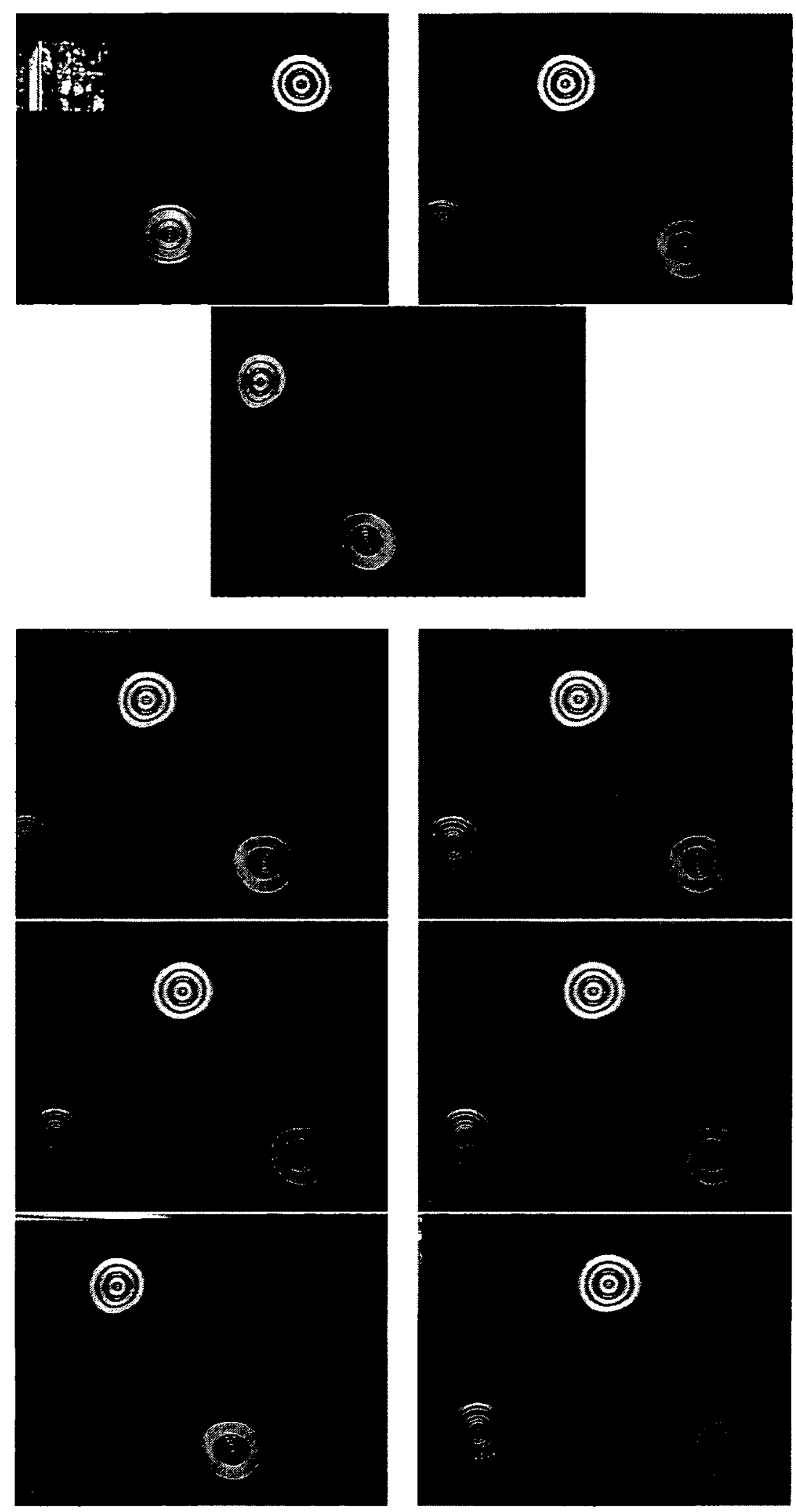

Figure A.-1: $4 \mathrm{~cm}$ markers on black background 


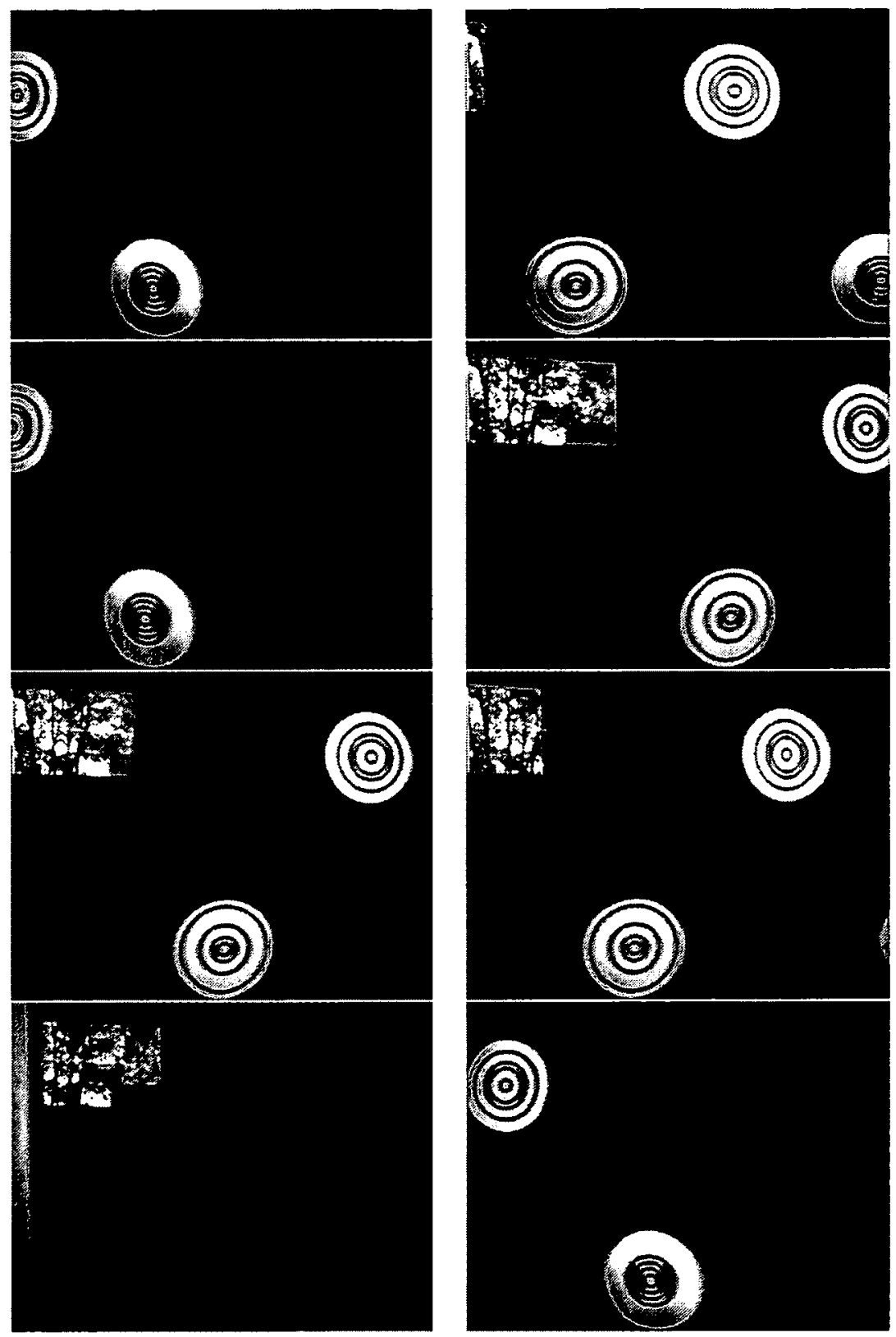

Figure A.-1: $6 \mathrm{~cm}$ markers on black background

Figure A.0: All 38 images of the results of marker design tests 


\section{Appendix B}

The coordinates of all marker in the sphere frame.

143.0972 .59610 .12

$267.94-192.29-597.903$

$3204.66-34.60-592.634$

$4130.46159 .86-589.275$

$5297.35150 .28-511.09$

$6351.73-45.37-487.96$

$7212.34-197.75-528.00$

$8-69.08-334.68-515.759$

$9375.32-236.64-404.52$.

$10494.86-79.43-336.30$

$11440.12103 .01-404.66$

$12357.29304 .71-400.99$

$13167.42318 .90-500.60$

$14488.65-259.00-232.11$

$15560.7486 .31-222.80$

$16493.12258 .82-257.24$

$17215.52-375.52-414.86$

$18362.67-393.76-265.39$

$1939.10-315.87-511.70$ 
$2031.82-469.97-371.70$

$21 \quad 185.82-506.55-254.87$

$22578.16-103.82-146.06$

$23607.4963 .07-20.38$

$24315.89-497.65-89.39$

$25120.56-580.73-68.10$

$26229.70-535.44103 .35$

$27410.27-421.7186 .56$

$28470.85-357.14-83.69$

$29536.28-258.1890 .63$

$30585.25-131.2367 .58$

$31431.99-317.14269 .08$

$32302.34-434.34272 .32$

$3325.65-580.94118 .14$

$34129.09-503.46289 .31$

35586.3931 .02191 .80

$36563.92231 .70-72.92$

$37361.11440 .19-244.32$

$38478.34373 .99-85.68$

39557.52230 .57113 .19

40490.32194 .96319 .13

$41511.28-156.48262 .06$

42476.6019 .52376 .08

43446.47374 .06214 .07

$44338.03519 .51-55.05$

$45221.35460 .44-350.79$

$46 \quad 429.27434 .46 \quad 119.29$ 
47349.68318 .17402 .16 48372.90119 .90473 .25 $49389.33-161.79436 .91$ 50313.6821 .55520 .93 $5128.61453 .85-421.89$ $5275.59566 .76-249.08$ $53267.17540 .13-159.84$ $54102.93614 .65-49.71$ $55-124.09530 .91-301.22$ $56-71.15611 .38-111.96$ 57260.93567 .1146 .60 58108.05597 .54161 .11 $59-276.98538 .98-153.02$ $60-176.56386 .56-453.49$ $61-327.10434 .35-309.38$ $62-363.20268 .62-435.30$ $63-492.26297 .76-267.96$ $64-426.24445 .32-127.22$ $65-213.44585 .8945 .31$ $66-40.55619 .6994 .11$ $\begin{array}{llll}67 & -404.43 & 483.91 & 16.17\end{array}$ $68-324.40498 .17211 .11$ $69-496.07367 .35175 .46$ $70-115.54566 .65233 .43$

$71-568.18291 .53-68.12$ $72-217.60446 .77384 .98$ $73-385.57359 .97358 .97$ 
7457.44512 .00351 .55

$75-534.21206 .74309 .50$

$76-609.98197 .63116 .29$

$77-509.65118 .06-367.88$

$78-607.12127 .62-190.30$

$79-646.49105 .6917 .34$

$80-484.03-80.15-414.25$

$81-362.2573 .92-507.89$

$82-398.72181 .33473 .74$

$83-231.55319 .32494 .46$

$84-93.38424 .70447 .22$

85244.14510 .15239 .82

$86-605.80-30.60-245.98$

$87-547.95-226.87-276.08$

$88-635.72-171.10-92.42$

$89-656.5735 .80-57.15$

$90-618.0822 .13237 .99$

$91-650.73-97.80103 .76$

$92-402.08-267.23-422.44$

$93-437.87-401.99-259.86$

$94-559.86-341.23-98.06$

$95-592.86-295.9968 .26$

$96-423.58-491.47-66.95$

$97-520.34-400.8498 .73$

$98-576.22-210.30264 .29$

$99-357.63-520.56131 .22$

$100-429.68-401.12286 .60$ 
$101-339.52-166.90460 .94$

$102-150.81-449.52323 .83$

$103-215.55-301.65451 .93$

$104-211.22-407.01-379.98$

$105-215.75-509.52-193.36$

$106-156.58-557.47-21.52$

$107-77.83-539.55176 .18$

$108-21.63-350.16474 .44$

$10931.004-533.934306 .879$

$110152.23-397.90423 .29$

$111330.79-282.62416 .22$

$11260.39-258.60543 .99$

$113199.30-166.13546 .41$

$114-155.56-282.73519 .89$

$115-500.50-43.18394 .02$

116193.41224 .65538 .36

117135.28363 .11479 .92

118110.3015 .31605 .49

$119-1.79236 .54572 .40$

$120-342.79-47.28527 .96$

$121-416.78191 .239452 .565$

$122-112.43-11.26608 .45$

$123-199.99125 .61580 .06$

$124-186.2907-158.8303566 .1565$

125000

126000

$127-12.97-550.52-228.68$ 
